\title{
MULTIVARIATE BOUNDED VARIATION FUNCTIONS OF JORDAN-WIENER TYPE
}

\author{
ALEXANDER BRUDNYI AND YURI BRUDNYI
}

\begin{abstract}
We introduce and study spaces of multivariate functions of bounded variation generalizing the classical Jordan and Wiener spaces. Multivariate generalizations of the Jordan space were given by several prominent researchers but each of them preserved only some special properties of the space used further in few selected applications. Unlike this the multivariate generalization of the Jordan space presented in this paper preserves all known and reveals some previously unknown properties of the space. These, in turn, are special cases of the basic properties of the introduced spaces proved in the paper. They, in particular, include results on discontinuity sets and pointwise differentiability of the bounded variation functions and their Luzin type and $C^{\infty}$ approximation. Moreover, the second part of the paper presents results on Banach structure of function spaces of bounded variation, namely, atomic decomposition and constructive characterization of their predual spaces and then constructive characterization of preduals of the last ones and following from here the so-called two-stars theorems relating second duals of separable subspaces of "vanishing variation" to the (nonseparable) spaces of bounded variation.
\end{abstract}

\section{INTRODUCTION}

1.1. Main Concept (Motivation). The paper is devoted to the study of a new family of multivariate function spaces generalizing the classical Jordan-Wiener spaces $\left\{V_{p}\right\}_{1 \leq p \leq \infty}$. A generic space of the family is denoted by $\dot{V}_{p}^{k}\left(Q^{d}\right)$, where $k \in \mathbb{N}, 1 \leq p<\infty$ and $Q^{d}$ is a $d$-dimensional closed cube; in what follows, without loss of generality we take

$$
Q^{d}:=[0,1]^{d} .
$$

The space consists of bounded on $Q^{d}$ functions with bounded $(k, p)$-variation denoted by $|f|_{V_{p}^{k}}$. To introduce and motivate this notion we use an equivalent form of the classical $p$-variation given for a function $f \in \ell_{\infty}[0,1]$ by

$$
|f|_{V_{p}}:=\sup _{\pi}\left(\sum_{I \in \pi} \operatorname{osc}(f ; I)^{p}\right)^{\frac{1}{p}}
$$

2010 Mathematics Subject Classification. Primary 26B30. Secondary 46 E35.

Key words and phrases. Spaces of bounded variation, oscillation, local polynomial approximation, packing, smoothness, signed Borel measure, atom, chain, duality, predual space, two-stars theorem.

Research of the first author is supported in part by NSERC. 
here $\pi$ is a packing, i.e., a set of pairwise nonoverlapping 1 closed intervals $I \subset[0,1]$ and

$$
\operatorname{osc}(f ; I):=\sup _{x, y \in I}|f(x)-f(y)|
$$

is the oscillation of $f$ on $I$.

The reader can easily see that (1.2) is equivalent to the classical Jordan definition with $\operatorname{osc}(f ; I)$ replaced by deviation $\delta(f ; I):=|f(b)-f(a)|$, where $I:=[a, b]$ and $\pi$ runs over partitions, i.e., coverings of $[0,1]$ by pairwise nonoverlapping intervals.

It seems to be natural to define multivariate spaces of Jordan-Wiener type by taking in (1.2) $f \in \ell_{\infty}\left(Q^{d}\right)$ and $\pi$ being a family of pairwise nonoverlapping closed subcubes $Q \subset Q^{d}$. However, assuming boundedness of such variation we obtain the family $\left\{\ell_{p}\left(Q^{d}\right)\right\}_{1 \leq p<\infty}$ that has no relation to the required function spaces of bounded variation.

To overcome this obstacle we enlarge the order of difference in (1.3) using a function of a cube $Q \mapsto \operatorname{osc}_{k}(f ; Q), Q \subset Q^{d}$, given by

$$
\operatorname{osc}_{k}(f ; Q):=\sup _{h>0}\left\{\left|\Delta_{h}^{k} f(x)\right|: x+j h \in Q ; j=0, \ldots, k\right\}
$$

here as usual

$$
\Delta_{h}^{k} f:=\sum_{j=0}^{k}(-1)^{k-j}\left(\begin{array}{l}
k \\
j
\end{array}\right) f(\cdot+j h) .
$$

The family of spaces $\dot{V}_{p}^{k}\left(Q^{d}\right)$ obtained in this way is the main object of our study.

Remark 1.1. The definition of $\dot{V}_{p}^{k}\left(Q^{d}\right)$ is meaningful only in the case of $k \geq \frac{d}{p}$; otherwise, $\dot{V}_{p}^{k}\left(Q^{d}\right)=\ell_{p}\left(Q^{d}\right)$.

To illustrate the results of the present paper we briefly discuss their special cases for the space $\dot{V}_{1}^{d}\left(Q^{d}\right)$ that may be regarded as a $d$-dimensional analog of Jordan space $V\left(=\dot{V}_{1}^{1}\left(Q^{1}\right)\right.$ in our notation). We justify its role as a "genuine" analog of Jordan's space for $d \geq 2$ by comparing its properties presented below with those for $d=1$.

In what follows, $\dot{V}_{1}^{d}\left(Q^{d}\right)$ is denoted for brevity by $\dot{V}\left(Q^{d}\right)$.

(a) For every $d \geq 1$, functions of $\dot{V}\left(Q^{d}\right)$ have at most countable sets of discontinuities, see Theorem 2.1.

(b) For every $d \geq 1$, functions of $\dot{V}\left(Q^{d}\right)$ are $d$-differentiable in the Peano sens 2 almost everywhere (a.e.).

In particular, this implies for $d=1$ the classical Lebesgue differentiability theorem, see Theorem 2.15](a).

(c) Given $f \in \dot{V}\left(Q^{d}\right), d \geq 1$, and $\varepsilon \in(0,1)$ there is a function $f_{\varepsilon} \in C^{d}\left(Q^{d}\right)$ such that

$$
\operatorname{mes}_{d}\left\{x \in Q^{d}: f(x) \neq f_{\varepsilon}(x)\right\}<\varepsilon,
$$

see Theorem 2.11,

\footnotetext{
${ }^{1}$ Two subsets of a topological space are nonoverlapping if their interiors are disjoint.

${ }^{2}$ See the corresponding definition in Section 2.2 .
} 
(d) Let $A C\left(Q^{d}\right), d \geq 1$, be a closed subspace of $\dot{V}\left(Q^{d}\right)$ consisting of functions $f \in \ell_{\infty}\left(Q^{d}\right)$ satisfying

$$
\lim _{|\pi| \rightarrow 0} \sum_{Q \in \pi} \operatorname{osc}_{k}(f ; Q)=0 ;
$$

here $|\pi|:=\sum_{Q \in \pi}|Q|$ and $|S|$ is a $d$-measure of $S \subset \mathbb{R}^{d}$.

Then it is true that

$$
A C\left(Q^{d}\right)=\dot{W}_{1}^{d}\left(Q^{d}\right)
$$

where hereafter $\dot{W}_{p}^{k}\left(Q^{d}\right)$ stands for the homogeneous Sobolev space of order $k$ over $L_{p}\left(Q^{d}\right)$.

Moreover, every $f \in A C\left(Q^{d}\right)$ is restored by some integral operator up to an additive term being a polynomial of degree $d-1$ via the distributional gradient $\nabla^{d} f:=\left(D^{\alpha} f\right)_{|\alpha|=d}$, see Corollary 2.20.

For $d=1$, this gives the so-called Fundamental Theorem of Calculus by Lebesgue-Vitali.

(e) Let $N \dot{V}\left(Q^{d}\right), d \geq 1$, be the normalized part of $\dot{V}\left(Q^{d}\right)$ so that

$$
\dot{V}\left(Q^{d}\right)=N \dot{V}\left(Q^{d}\right) \oplus \ell_{1}\left(Q^{d}\right)
$$

see Section 2.1 below.

Then it is true that

$$
N \dot{V}\left(Q^{d}\right)=B \dot{V}^{d}\left(Q^{d}\right)
$$

where $B \dot{V}^{k}\left(Q^{d}\right)$ is the Sobolev space of order $k$ whose distributional $k$-th derivatives are bounded Borel measures, see Theorem 2.18(c).

For $d=1$, this gives the classical Hardy-Littlewood theorem HL-28] as $B \dot{V}^{1}\left(Q^{1}\right)$ coincides with Lipschitz space over $L_{1}\left(Q^{1}\right)$.

(f) If a sequence $\left\{f_{n}\right\}_{n \in \mathbb{N}} \subset \dot{V}\left(Q^{d}\right), d \geq 1$, is such that

$$
\sup _{n \in \mathbb{N}}\left|f_{n}\right|_{V\left(Q^{d}\right)}<\infty
$$

then for some sequences $J \subset \mathbb{N}$ of integers and $\left\{p_{n}\right\}_{n \in J}$ of polynomials of degree $d-1$ on $\mathbb{R}^{d}$ the sequence $\left\{f_{n}+p_{n}\right\}_{n \in J}$ pointwise converges to some $f \in \dot{V}\left(Q^{d}\right)$ and, moreover,

$$
|f|_{V\left(Q^{d}\right)} \leq \underline{\lim }_{n \in J}\left|f_{n}\right|_{V\left(Q^{d}\right)}
$$

For $d=1$, this gives the classical Helly selection theorem.

The variety of useful properties of the space $\dot{V}\left(Q^{d}\right)$ (more generally, spaces $\dot{V}_{p}^{k}\left(Q^{d}\right)$ ) allows their applicability to various fields of modern analysis including multivariate harmonic analysis, $N$-term approximation, the real interpolation of smoothness spaces. The corresponding results will be presented in the forthcoming papers.

Remark 1.2. There are two more spaces of the family, namely, $\dot{V}_{d}^{d}\left(Q^{d}\right)$ and $\dot{V}_{d}^{1}\left(Q^{d}\right)$ which for $d=1$ coincide with the space $V[0,1]$. However, they do not possess almost all of the above formulated properties for $\dot{V}\left(Q^{d}\right)$ (e.g., if $d \geq 2$, then $\dot{V}_{d}^{d}\left(Q^{d}\right)$ contains nowhere differentiable functions). 
1.2. Historical Remarks. An important role of the space $V$ in univariate function theory attracted several prominent researchers to the problem of its multivariate generalization. The introduced notions single out multivariate function spaces preserving only some particular properties of the Jordan space. These properties, in turn, were used to solve a specific problem of multivariate analysis.

In this paper we briefly discuss only those works that have been influenced further development of the multivariate theory.

(a) Vitali variation (associated with the names of Vitali [V-05], Lebesgue [Le-10], Vallée Poussen [VP-16]).

Let $[x, y] \subset \mathbb{R}^{d}, x=\left(x_{1}, \ldots, x_{d}\right), y=\left(y_{1}, \ldots, y_{d}\right)$, be a $d$-interval, i.e., the set

$$
[x, y]:=\left\{z=\left(z_{1}, \ldots, z_{d}\right) \in \mathbb{R}^{d}: x_{i} \leq z_{i} \leq y_{i}, i=1, \ldots, d\right\} .
$$

For a function $f \in \ell_{\infty}(I), I:=[x, y]$, we define the deviation

$$
\delta(f ; I)=\sum_{j_{1}=0}^{1} \cdots \sum_{j_{d}=0}^{1}(-1)^{j_{1}+\cdots+j_{d}} f\left(y_{1}+j_{1}\left(x_{1}-y_{1}\right), \ldots, y_{d}+j_{d}\left(x_{d}-y_{d}\right)\right) ;
$$

in particular, for $d=2$

$$
\delta(f ; I)=f\left(y_{1}, y_{2}\right)-f\left(y_{1}, x_{2}\right)-f\left(x_{1}, y_{2}\right)+f\left(x_{1}, x_{2}\right) .
$$

The Vitali variation is given for $f \in \ell_{\infty}\left(Q^{d}\right)$ by

$$
\operatorname{var}_{v} f:=\sup _{\pi}\left\{\sum_{I \in \pi}|\delta(f ; I)|\right\}
$$

where $\pi$ runs over all families of pairwise nonoverlapping $d$-intervals in $Q^{d}$.

The space of functions on $Q^{d}$ with bounded Vitali variations is denoted by $V_{v}\left(Q^{d}\right)$.

Remark 1.3. (a) In the original definition, see, e.g., AC-33], $\pi$ runs over all partitions of $Q^{d}$ by such $d$-intervals. Since

$$
\delta(f ; I)=\sum_{I^{\prime} \in \pi} \delta\left(f ; I^{\prime}\right),
$$

where $\pi$ is a partition of $I$ in nonoverlapping $d$-intervals $I^{\prime}$ by hyperplanes parallel to the coordinate ones, this definition is equivalent to (1.7).

(b) The deviations in (1.7) can be clearly replaced by oscillations; here

$$
\operatorname{osc}(f ; I):=\sup _{I^{\prime} \subset I}\left|\delta\left(f ; I^{\prime}\right)\right|,
$$

where supremum is taken over all $d$-subintervals of $I$.

Unlike affinity of their definitions the structure of functions from $V_{v}\left(Q^{d}\right)$ is essentially poorer than that for functions from $\dot{V}\left(Q^{d}\right)$. In particular, the former contains nonmeasurable functions and nowhere differentiable functions.

Nevertheless, the affinity leads to the following continuous embedding

$$
(\dot{V} \cap C)\left(Q^{d}\right) \subset V_{v}\left(Q^{d}\right) .
$$


It remains to present the only application of Vitali variation to prove the existence of the Riemann-Stiltjes type multiple integral $\int_{Q^{d}} f d g$ for $f \in C\left(Q^{d}\right)$ and $g \in V_{v}\left(Q^{d}\right)$. i.e., the existence of the limit

$$
\lim _{\Pi} \sum_{I \in \pi} f\left(x_{I}\right) \delta(g ; I)
$$

where $\pi$ is a partition of $Q^{d}$ in $d$-intervals by hyperplanes parallel to the coordinate ones, $x_{I} \in I$ and $\Pi=\{\pi\}$ is a net of such partitions with meshes $\sup _{I \in \pi}|I| \rightarrow 0$.

(b) Hardy-Krause variation.

This notion denoted by $\operatorname{var}_{h} f$ is defined via Vitali variation as follows.

Let $\left\{e^{i}\right\}_{1 \leq i \leq n}$ be the standard basis of $\mathbb{R}^{d}$ and $\hat{x} \in Q^{d}, \omega \subset\{1, \ldots, d\}$ be fixed. Then a partial function of $f \in \ell_{\infty}\left(Q^{d}\right)$ defined by $\hat{x}, \omega$ is given by

$$
f_{\hat{x}, \omega}:\left(x_{i}\right)_{i \in \omega} \mapsto f\left(\sum_{i \in \omega} x_{i} e^{i}+\sum_{i \notin \omega} \hat{x}_{i} e^{i}\right) ;
$$

this is clearly defined on the unit cube $Q^{\omega} \cong[0,1]^{|\omega|}$ of dimension $|\omega|$ in the subspace of $\mathbb{R}^{d}$ generated by vectors $e^{i}, i \in \omega$.

Now Hardy-Krause variation is given for $f \in \ell_{\infty}\left(Q^{d}\right)$ by

$$
\operatorname{var}_{h} f:=\sum_{\omega} \operatorname{var}_{v} f_{\omega}
$$

where $\omega$ runs over all nonempty subsets of $\{1, \ldots, d\}$.

The space of functions of bounded variation (1.9) is denoted by $V_{h}\left(Q^{d}\right)$.

The functions of this subspace of $V_{v}\left(Q^{d}\right)$ are of essentially better structure. In particular, every $f \in V_{h}\left(Q^{d}\right)$ has one-sided limits in each variable and is differentiable a.e. The former is the matter of definition and the latter is proved in [BH-32] for $d=2$ (the proof can be easily extended to all $d$ ).

The main application of Hardy-Krause variation is a generalization of Dirichlet-Jordan convergence criterion for multiple Fourier series, see [H-06] for $d=2$ and [MT-50] for $d>2$. For some modern development of these results in multivariate harmonic analysis, approximation theory and numerical computations see, e.g., [Te-015] and references therein.

(c) Tonelli variation.

Let $f \in \ell_{\infty}\left(Q^{d}\right)$ and $f_{x^{i}}: x_{i} \mapsto f(x), 0 \leq x_{i} \leq 1$, be a univariate partial function with $x^{i}:=\sum_{j \neq i} x_{j} e^{j}$ being fixed, $1 \leq i \leq d$.

Tonelli variation for this $f \in \ell_{\infty}\left(Q^{d}\right)$ is given by

$$
\operatorname{var}_{t} f:=\sum_{i=1}^{d} \int_{Q^{d-1}}\left(\operatorname{var} f_{x^{i}}\right) d x^{i}
$$

here the (Jordan) variations are assumed to be Lebesgue integrable.

This notion is introduced to characterize continuous functions on $Q^{d}$ whose graphs are rectifiable, see [T-26] for $d=2$ and proof in [S-37, Ch.5] that is easily extended to $d>2$. 
For integrable functions, Tonelli variation loses its geometric meaning, see [AC-34. The corresponding adaptation to this case was given by [Ce-36]; the modern version exploiting theory of distributions is presented in the books [Gi-84 and AFP-00]. The corresponding variation for $f \in L_{1}\left(Q^{d}\right)$ is given by

$$
\operatorname{var}_{Q^{d}} f:=\sum_{i=1}^{d} \operatorname{var}_{Q^{d}} \partial_{i} f
$$

where the distributional partial derivatives are assumed to be finite Borel measures.

The corresponding space is denoted by $B V\left(Q^{d}\right)$.

This definition is given in essence by De Giorgi [DG-54]; its equivalence to that of Tonelli-Cesari is proved in the papers [FKP-57]. In particular, for $f \in C\left(Q^{d}\right)$

$$
\operatorname{var}_{t} f \approx \operatorname{var} f
$$

with constants of equivalence depending only on $d$.

Nevertheless, there is some connection of this space with a space of the family $\left\{\dot{V}_{p}^{k}\left(Q^{d}\right)\right\}$. Actually, using the equivalent definition of $\underset{Q^{d}}{\operatorname{var} f}$ given for $f \in L_{1}\left(Q^{d}\right), d \geq 2$, by

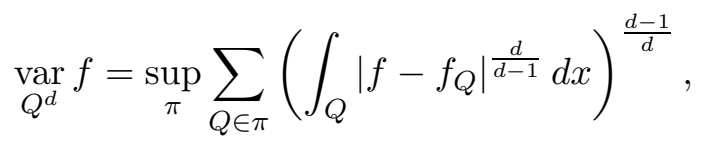

where $f_{Q}:=\frac{1}{|Q|} \int_{Q} f d x$, see [BB-18, Sec. 1.3.4], and then applying the Hölder inequality we have

$$
\underset{Q^{d}}{\operatorname{var} f \leq c(d)|f|_{V_{d}^{1}\left(Q^{d}\right)}}
$$

Finally, we present one more variation given by Kronrod [Kr-50] for $d=2$ and by Vitushkin [Vi-55] for all $d$.

It is given only for continuous functions on $Q^{d}$ by

$$
\operatorname{var}_{k v} f:=\int_{\mathbb{R}} H_{d-1}\left\{x \in Q^{d}: f(x)=t\right\} d t,
$$

where $H_{d-1}$ is the $(d-1)$-Hausdorff measure.

For $d=1$, this coincides with Jordan variation of $f$ by the Banach indicatrix theorem and for $d>1$

$$
\operatorname{var}_{k v} f \approx \operatorname{var}_{t} f
$$

with constants of equivalence depending only on $d$, see [Kr-50].

In fact, the definitions in the cited papers include also the definition of variations related to the sections of function graphs by planes of dimensions $1 \leq s \leq d-1$. For the applications of these characteristics to the geometry of sets, approximation theory and the 13th Hilbert problem, see [Vi-004]. 
1.3. Basic Definitions. In the presentation of the main objects of our study and everywhere below, we use the following:

Notation 1.4. $\mathbb{N}, \mathbb{Z}, \mathbb{R}$ denote the sets of natural, integer and real numbers, respectively. $\mathbb{R}^{d}$ is the $d$-dimensional Euclidean space of vectors $x=\left(x_{1}, \ldots, x_{d}\right), y, z$, etc.

If $S \subset \mathbb{R}^{d}$, then $\bar{S}$ denotes its closure and $\stackrel{\circ}{S}=\operatorname{int} S$ its interior.

The $d$-dimensional Lebesgue measure of $S$ is denoted by $|S|$. The characteristic function (indicator) of $S$ is denoted by $\chi_{S}$.

As above, $Q^{d}$ denotes the unit cube $[0,1]^{d} . Q, Q^{\prime}$, etc. denote subcubes of $\mathbb{R}^{d}$ homothetic to $Q^{d}$ (named further cubes or subcubes); $Q_{r}(x)$ denotes a cube centered at $x$ of sidelength $2 r$.

$\pi, \pi^{\prime}, \pi_{i}$, etc. denote packings, i.e., families of nonoverlapping cubes $Q \subset \mathbb{R}^{d}$.

$\Pi(S)$ (briefly, $\Pi$ for $S=Q^{d}$ ) denotes the set of packings in $S$ of finite cardinality contained in $S$.

$\mathcal{P}_{k}^{d}$ denotes the linear space of polynomials in $x \in \mathbb{R}^{d}$ of degree $k$, i.e., the linear hull of monomials $x^{\alpha},|\alpha| \leq k$, where

$$
x^{\alpha}:=\prod_{i=1}^{d} x_{i}^{\alpha_{i}}, \quad|\alpha|:=\sum_{i=1}^{d} \alpha_{i}, \quad \alpha \in \mathbb{Z}_{+}^{d} .
$$

$\ell_{p}(S), 1 \leq p \leq \infty, S \subset \mathbb{R}^{d}$, denotes the Banach space of functions $f: S \rightarrow \mathbb{R}$ defined by norm

$$
\|f ; S\|_{p}:=\left\{\sum_{x \in S}|f(x)|^{p}\right\}^{\frac{1}{p}}
$$

in particular,

$$
\|f ; S\|_{\infty}:=\sup _{S}|f| .
$$

All $f \in \ell_{p}(S)$ are clearly bounded on $S$ and their supports supp $f:=\{x \in S: f(x) \neq 0\}$ are at most countable for $p<\infty$.

$\ell_{p}^{\text {loc }}\left(\mathbb{R}^{d}\right)$ consists of functions on $\mathbb{R}^{d}$ whose traces to every compact subset $K$ belong to $\ell_{p}(K)$.

$C(S), S \subset \mathbb{R}^{d}$, denotes the Banach space of the uniformly continuous functions $f: S \rightarrow \mathbb{R}$ equipped with norm $\|f\|_{C(S)}:=\sup _{S}|f|$.

$C^{\infty}(S)$ denotes the linear space of traces $\left.f\right|_{S}$ of functions $f \in C^{\infty}\left(\mathbb{R}^{d}\right)$.

Let $X, Y$ be linear (semi-) normed vector spaces. We write

$$
X \hookrightarrow Y
$$

if there is a linear continuous injection of $X$ into $Y$, and replace $\hookrightarrow$ by $\subset$ if $X$ is a linear subspace of $Y$ and the natural embedding operator $X \rightarrow Y$ is continuous.

Further, we say that these spaces are isomorphic and write

$$
X \cong Y
$$


if $X \hookrightarrow Y$ and $Y \hookrightarrow X$ and composition $X \rightarrow X$ of these continuous injections is the identity map; moreover, we write

$$
X=Y
$$

if, in addition, they coincide as linear spaces, hence, have equivalent (semi-) norms.

Moreover, spaces $X$ and $Y$ are said to be isometrically isomorphic if the injections in (1.14) are of norm 1 . We write in this case

$$
X \equiv Y .
$$

Finally, we denote by $B(X)$ the closed unit ball of a (semi-) normed space $X$, i.e.,

$$
B(X):=\left\{x \in X:\|x\|_{X} \leq 1\right\} .
$$

Now we present the basic notions of the paper.

1.3.1. Local polynomial approximation. In the sequel, we exploit an essential more suitable for applications definition of $(k, p)$-variation. The main point here is the replacement of $\mathrm{osc}_{k}$ in the initial definition by a function of cube denoted by $E_{k}(f ; \cdot)$ and called local polynomial approximation, see Definition 1.5 below. The relation between these two set-functions is given for $I \subset \mathbb{R}$ by the equivalence Wh-59]

$$
E_{k}(f ; I) \approx \operatorname{osc}_{k}(f ; I),
$$

where the constants of equivalence depend only on $k$.

The general result of this kind proved below, see Theorem 6.1, shows that these two definitions of $(k, p)$-variation are equivalent. However, the second definition is more suitable for applications since it relies on various techniques of local approximation theory and so is easier to apply to the study of the Banach structure of $V_{p}^{k}\left(Q^{d}\right)$ spaces and their elements. (For instance, the fact that $(k, p)$-variation of $f \in \ell_{\infty}\left(Q^{d}\right)$ is zero iff $f$ is a polynomial of degree $k-1$ trivially follows from the second definition but is highly nontrivial in the framework of the first one.)

Definition 1.5. Local polynomial approximation of order $k \in \mathbb{N}$ is a function

$$
E_{k}:(f, S) \mapsto \mathbb{R}_{+}, \quad f \in \ell_{\infty}^{\text {loc }}\left(\mathbb{R}^{d}\right), S \subset \mathbb{R}^{d},
$$

given by

$$
E_{k}(f ; S):=\inf _{m \in \mathcal{P}_{k-1}^{d}}\|f-m ; S\|_{\infty}
$$

This function possesses several important properties, see, in particular, [Br1-94, §2], that will be used in the forthcoming proofs and mostly are proved there.

1.3.2. ( $k, p)$-variation. Using the previous notion we introduce a set-function $\operatorname{var}_{p}^{k}(f ; S)$, $k \in \mathbb{N}, 1 \leq p<\infty$, called $(k, p)$-variation. 
Definition 1.6. $(k, p)$-variation of a locally bounded function $f \in \ell_{\infty}^{\text {loc }}\left(\mathbb{R}^{d}\right)$ on a bounded set $S \subset \mathbb{R}^{d}$ is given by

$$
\operatorname{var}_{p}^{k}(f ; S):=\sup _{\pi \in \Pi(S)}\left(\sum_{Q \in \pi} E_{k}(f ; Q)^{p}\right)^{\frac{1}{p}}
$$

this equals 0 if $\stackrel{\circ}{S}=\emptyset$.

It is the matter of definition to verify the next properties of the object introduced.

Proposition 1.7. (Subadditivity) If $\left\{S_{i}\right\}_{i \in \mathbb{N}}$ is a sequence of disjoint subsets in $\mathbb{R}^{d}$, then

$$
\left(\sum_{i=1}^{\infty} \operatorname{var}_{p}^{k}\left(f ; S_{i}\right)^{p}\right)^{\frac{1}{p}} \leq \operatorname{var}_{p}^{k}\left(f ; \bigcup_{i=1}^{\infty} S_{i}\right) .
$$

(Lower semicontinuity) If a sequence $\left\{f_{i}\right\}_{i \in \mathbb{N}} \subset \ell_{\infty}(S)$ converges in this space to a function $f$, then

$$
\operatorname{var}_{p}^{k}(f ; S) \leq \varliminf_{i \rightarrow \infty} \operatorname{var}_{p}^{k}\left(f_{i} ; S\right)
$$

(Monotonicity) The function

$$
(k, p, S) \mapsto \operatorname{var}_{p}^{k}(f ; S)
$$

is nondecreasing in $S$ and nonincreasing in $k, p$.

1.3.3. $V_{p}^{k}$ spaces. Now we consider $\operatorname{var}_{p}^{k}$ as a function of $f$. Using again its definition we have the following:

Proposition 1.8. Let $S \subset \mathbb{R}^{d}$ be the closure of a domain (open connected set). Then the function

$$
f \mapsto \operatorname{var}_{p}^{k}(f ; S) \in[0, \infty], \quad f \in \ell_{\infty}^{\text {loc }}(S),
$$

satisfies properties of a seminorm and equals 0 iff $\left.f \in \mathcal{P}_{k-1}^{d}\right|_{S}$.

Now we present the main object of our study.

Definition 1.9. $\dot{V}_{p}^{k}\left(Q^{d}\right)$ is a linear space of functions $f \in \ell_{\infty}\left(Q^{d}\right)$ whose $(k, p)$-variation

$$
|f|_{V_{p}^{k}}:=\operatorname{var}_{p}^{k}\left(f ; Q^{d}\right), \quad f \in \dot{V}_{p}^{k}\left(Q^{d}\right)
$$

is finite.

By Proposition $1.8\left(\dot{V}_{p}^{k}(S),|\cdot|_{V_{p}^{k}}\right)$ is a seminormed space with $\left.\mathcal{P}_{k-1}^{d}\right|_{Q^{d}}$ being its nullspace. The standard argument shows that $\dot{V}_{p}^{k}(S)$ is complete.

Stipulation 1.10. Since $Q^{d}:=[0,1]^{d}$ is fixed, throughout the paper we remove it from notations writing, e.g., $\dot{V}_{p}^{k}, C^{\infty}, \mathcal{P}_{k-1}^{d}$ instead of $\dot{V}_{p}^{k}\left(Q^{d}\right), C^{\infty}\left(Q^{d}\right),\left.\mathcal{P}_{k-1}^{d}\right|_{Q^{d}}$. 
In some cases, it will be more appropriate to use a seminorm on $\dot{V}_{p}^{k}$ defined by replacing in (1.19) $E_{k}(f ; Q)$ by $\operatorname{osc}_{k}(f ; Q), Q \in \pi$. The latter is given for $f \in \ell_{\infty}^{\text {loc }}\left(\mathbb{R}^{d}\right)$ and a bounded subset $S \subset \mathbb{R}^{d}$ by

$$
\operatorname{osc}_{k}(f ; S):=\sup _{h \in \mathbb{R}^{d}}\left\{\left|\Delta_{h}^{k} f(x)\right|: x+j h \in S, j=0, \ldots, k\right\},
$$

cf. definition (1.4) .

Our work is divided into two parts where the first one studies pointwise behaviour of $\dot{V}_{p}^{k}$ functions while the second is focused on the duality structure of a Banach space $V_{p}^{k}$ associated to $\dot{V}_{p}^{k}$. Among different equivalent presentations of this space we prefer the quotient of $\dot{V}_{p}^{k}$ by its null-space. Hence, we define an associated to $\dot{V}_{p}^{k}$ Banach space by setting

$$
V_{p}^{k}:=\dot{V}_{p}^{k} / \mathcal{P}_{k-1}^{d}
$$

and denoting by $\|\cdot\|_{V_{p}^{k}}$ its (factor)-norm.

If $\stackrel{\circ}{f} \in V_{p}^{k}$ is a factor-class $\{f\}+\mathcal{P}_{k-1}^{d}, f \in \dot{V}_{p}^{k}$, then clearly

$$
\|\stackrel{\circ}{f}\|_{V_{p}^{k}}=|f|_{V_{p}^{k}} .
$$

By this reason, we call elements of $V_{p}^{k}$ functions and denote them by $f, g$, etc. by

In both parts of the work, the essential role plays a numerical characteristic of $\dot{V}_{p}^{k}$ given

$$
s\left(\dot{V}_{p}^{k}\right):=\frac{d}{p}
$$

and called smoothness.

For instance, a function $f \in \dot{V}_{p}^{k}$ satisfies the Lipschitz condition of order $s\left(:=s\left(\dot{V}_{p}^{k}\right)\right)$ a.e. if $0<s<k$, has the $k$-th Peano differential a.e. if $s=k$ and equals a polynomial of degree $k-1$ outside of at most countable subset of $Q^{d}$ if $s>k$, see Theorems 2.1, 2.15 below.

Moreover, in the second part we essentially use a closed separable subspace 3 of $\dot{V}_{p}^{k}$ denoted by $\dot{\mathrm{V}}_{p}^{k}$ (respectively, $\mathrm{v}_{p}^{k} \subset V_{p}^{k}$ ) given by

$$
\dot{\mathrm{V}}_{p}^{k}:=\operatorname{clos}\left(C^{\infty} \cap \dot{V}_{p}^{k}, \dot{V}_{p}^{k}\right) .
$$

1.4. Outline of the Paper. The paper is organized as follows.

Section 2 devoted to the formulations of the main results is composed of four subsections. Subsection 2.1 contains results describing the structure of $\dot{V}_{p}^{k}$ function spaces.

Subsection 2.2 presents several results describing relations between $\dot{V}_{p}^{k}$ and the Lipschitz spaces over $\ell_{\infty}$ and $L_{p}$ of order $s:=\frac{d}{p} \in(0, k]$. These include Luzin's type approximation of $\dot{V}_{p}^{k}$ functions by Lipschitz and $C^{k}$ functions, the Lebesgue type theorem on their pointwise differentiability a.e. of order $s$ and the Hardy-Littlewood type theorem on two-sided

\footnotetext{
${ }^{3}$ As we will see, space $\dot{V}_{p}^{k}$ is nonseparable.
} 
embeddings connecting $\dot{V}_{p}^{k}$ with Lipschitz-Besov spaces over $L_{p}$. As a consequence, the latter gives a multivariate generalization of the Lebesgue-Vitali Fundamental Theorem of Calculus.

Subsection 2.3 introduces a predual to $V_{p}^{k}$ Banach space denoted by $U_{p}^{k}$ and describes its Banach structure and the relation $\left(U_{p}^{k}\right)^{*} \equiv V_{p}^{k}$.

Subsection 2.4 contains the so-called two-stars theorem relating the second dual of the space $\mathrm{V}_{p}^{k}$ to the nonseparable space $V_{p}^{k}$. The formulations preceding to that result describe measure theoretic properties of some dense subspace of $U_{p}^{k}$ and following from them and the F. Riesz representation theorem the relation $\left(\mathrm{v}_{p}^{k}\right)^{*} \cong U_{p}^{k}$.

The remaining sections contain the proofs of the formulated results.

\section{Formulation of the Main Results}

2.1. Structure Properties of $\dot{V}_{p}^{k}$ Functions. Our first result shows that $\dot{V}_{p}^{k}$ functions initially assumed to be only bounded (not necessarily measurable) have much finer structure.

Theorem 2.1. Let $f$ be $a \dot{V}_{p}^{k}$ function of smoothness s. Then the following is true.

(a) If $d=1$, then $f$ has one-sided limits at each $f \in[0,1]$.

(b) If $d \geq 2$, then $f$ has a limit at each point $x \in Q^{d}$.

In both cases, $f$ has at most countable set of discontinuity points.

(c) If $s>k$, then the vector space $\dot{V}_{p}^{k}$ is the direct sum of subspaces $\ell_{p}$ and $\mathcal{P}_{k-1}^{d}$.

Assertion (c) implies that for $s>k$ the Banach spaces $V_{p}^{k}$ and $\ell_{p}$ are isomorphic. Since this case is not of interest, we assume in the sequel that $\dot{V}_{p}^{k}$ satisfies the condition

$$
s\left(\dot{V}_{p}^{k}\right):=\frac{d}{p} \leq k .
$$

Now let $f \in \dot{V}_{p}^{k}$ and $\hat{f}$ be given by

$$
\hat{f}(x):=\lim _{y \rightarrow x} f(y), \quad x \in Q^{d}
$$

for $d \geq 2$, and by

for $d=1$.

$$
\hat{f}(x):=\left\{\begin{array}{cc}
\frac{1}{2}\left(f\left(x^{-}\right)+f\left(x^{+}\right)\right), & 0<x<1, \\
f\left(0^{+}\right), & x=0, \\
f\left(1^{-}\right), & x=1,
\end{array}\right.
$$

Corollary 2.2. (a) The linear map $P$ on $\dot{V}_{p}^{k}$ sending $f$ to $\hat{f}$ is a projection of norm 1 on a closed subspace denoted by $N \dot{V}_{p}^{k} \subset \dot{V}_{p}^{k}$. 
By the definition, $N \dot{V}_{p}^{k}=C \cap \dot{V}_{p}^{k}$ if $d \geq 2$ and consists of functions $f \in \dot{V}_{p}^{k}$ such that $f(x)=\frac{1}{2}\left(f\left(x^{-}\right)+f\left(x^{+}\right)\right), x \in(0,1)$, if $d=1$.

(b) The kernel of $P$ coincides as a linear space with $\ell_{p}$ and, moreover,

$$
|f|_{V_{p}^{k}} \leq\|f\|_{p} \leq 2|f|_{V_{p}^{k}}, \quad f \in \operatorname{ker}(P) .
$$

In particular, the space $\left(\operatorname{ker}(P),|\cdot|_{V_{p}^{k}}\right)$ is Banach.

(c) $\dot{V}_{p}^{k}$ is isomorphic to the direct sum $N \dot{V}_{p}^{k} \oplus \ell_{p}$.

Hereafter, the direct sum of (semi-) normed spaces $X, Y$ is defined by a (semi-) norm given for $(x, y) \in X \times Y$ by

$$
\|(x, y)\|:=\|x\|_{X}+\|y\|_{Y} .
$$

Now we present two results on $C^{\infty}$ approximation of $\dot{V}_{p}^{k}$ functions. The first of them concerns approximation in the weak ${ }^{*}$ topology of $\dot{V}_{p}^{k}$ induced by duality $\ell_{1}^{*}=\ell_{\infty}$. In its formulation and proof we use integrability on $Q^{d}$ of functions $f \in \dot{V}_{p}^{k}$, see Theorem 2.1.

Theorem 2.3. For every function $f \in \dot{V}_{p}^{k}$ there is a sequence of $C^{\infty}$ functions $\left\{f_{n}\right\}_{n \in \mathbb{N}}$ such that

$$
\varlimsup_{n \rightarrow \infty}\left\|f_{n}\right\|_{\infty} \leq 3\|f\|_{\infty} \quad \text { and } \quad \varlimsup_{n \rightarrow \infty}\left|f_{n}\right|_{V_{p}^{k}} \leq 5|f|_{V_{p}^{k}}
$$

and, moreover, for every finite signed Borel measure on $Q^{d}$,

$$
\lim _{n \rightarrow \infty} \int_{Q^{d}}\left(f-f_{n}\right) d \mu=0 .
$$

Aa a consequence of the theorem we obtain the following generalization of the classical Helly selection theorem.

Corollary 2.4. For every bounded sequence $\left\{f_{n}\right\}_{n \in \mathbb{N}} \subset \dot{V}_{p}^{k}$ there are sequences $J \subset \mathbb{N}$ and $\left\{p_{n}\right\}_{n \in J} \subset \mathcal{P}_{k-1}^{d}$ such that the sequence $\left\{f_{n}+p_{n}\right\}_{n \in J}$ pointwise converges to some $f \in \dot{V}_{p}^{k}$ and, moreover,

$$
|f|_{V_{p}^{k}} \leq \varliminf_{n \in J}\left|f_{n}\right|_{V_{p}^{k}}
$$

The next result used in the proof of the second approximation result relates spaces $\dot{V}_{p}^{k}$ and $N \dot{V}_{p}^{k}$ to the space $\dot{V}_{p \infty}^{k}$ defined in the very same way as $\dot{V}_{p}^{k}$ but with $\ell_{\infty}$ replaced by $L_{\infty}$ and with the respectively modified variation, see Definition [1.9. In its formulation, $L: \dot{V}_{p}^{k} \rightarrow L_{\infty}$ is a linear map sending a function from $\dot{V}_{p}^{k}$ to its equivalence class in $L_{\infty}$.

Theorem 2.5. (a) range $(L)=\dot{V}_{p \infty}^{k}$ and the operator $L: \dot{V}_{p}^{k} \rightarrow \dot{V}_{p \infty}^{k}$ has norm 1 ;

(b) $\operatorname{ker}(L)=\operatorname{ker}(P)=\ell_{p} \subset \dot{V}_{p}^{k}$.

(c) $L$ maps $N \dot{V}_{p}^{k}$ isometrically onto $\dot{V}_{p \infty}^{k}$.

The second approximation result characterizes $\dot{V}_{p}^{k}$ functions admitting $C^{\infty}$ approximation, i.e., functions of the subspace $\dot{\mathrm{V}}_{p}^{k}$. 
Theorem 2.6. Let a function $f \in \dot{V}_{p}^{k}$ and smoothness $s$ of $\dot{V}_{p}^{k}$ satisfy the condition

$$
k>s(:=d / p) \text {. }
$$

Then $f \in \dot{\mathrm{V}}_{p}^{k}$, i.e., $f$ is approximated by $C^{\infty}$ functions in $\dot{V}_{p}^{k}$, see (1.24), iff

$$
\lim _{\varepsilon \rightarrow 0} \sup _{d(\pi) \leq \varepsilon}\left(\sum_{Q \in \pi} E_{k}(f ; Q)^{p}\right)^{\frac{1}{p}}=0 ;
$$

hereafter $d(\pi):=\sup _{Q \in \pi}|Q|$.

Remark 2.7. In fact, we will see that $C^{\infty} \subset \dot{V}_{p}^{k}$ and $\dot{\mathrm{v}}_{p}^{k} \subset C$ for $s \leq k$.

2.2. Relations Between Multivariate $B V$ Spaces and Lipschitz-Besov Spaces. Embeddings connected Jordan-Wiener spaces $B V_{p}$ and Lipschitz spaces over $L_{p}$ were discovered by Hardy and Littlewood [HL-28] and then intensively studied by many researches, see, in particular, Ge-54, KL-09] and references therein.

Below we present relations of these and some other types whose proofs however require essentially new tools including Sobolev type embeddings, interpolation space results and multivariate generalization of Whitney type inequality.

We begin with the last result where we deal with the definition of $(k, p)$-variation via $k$-oscillation, see (1.21).

Theorem 2.8. For every $f \in \ell_{\infty}^{\mathrm{loc}}\left(\mathbb{R}^{d}\right)$ the following two-sided inequality

$$
|f|_{V_{p}^{k}} \approx \sup _{\pi \in \Pi}\left\{\sum_{Q \in \pi} \operatorname{osc}_{k}(f ; Q)^{p}\right\}^{\frac{1}{p}}
$$

holds with constants of equivalence depending only on $k, d$.

Hereafter $\Pi$ stands for the set of all finite packings in $Q^{d}$.

Remark 2.9. The main point of this result is a generalized Whitney inequality given by

$$
E_{k}(f ; Q) \leq c(k, d) \operatorname{osc}_{k}(f ; Q) .
$$

The result is known for functions from $L_{p}^{\text {loc }}\left(\mathbb{R}^{d}\right), 1 \leq p \leq \infty$, and many other Banach function spaces [Br1-70] but its proof exploits tools of measure theory which is impossible for the derivation of (2.6).

The next result shows that a $V_{p}^{k}$ function of smoothness $s \in(0, k]$ "almost" coincides with a Lipschitz function of the same smoothness. Here Lipschitz space $\Lambda^{k, s}$ consists of continuous on $Q^{d}$ functions satisfying for $x, x+k h \in Q^{d}$ the condition

$$
\left|\Delta_{h}^{k} f(x)\right| \leq c\|h\|^{s},
$$

where $\|h\|:=\max _{1 \leq i \leq d}\left|h_{i}\right|, h=\left(h_{1}, \ldots, h_{d}\right) \in \mathbb{R}^{d}$.

The equality

$$
|f|_{\Lambda^{k, s}}:=\inf c
$$


defines a Banach seminorm of $\Lambda^{k, s}$. Moreover, $\Lambda^{k, s}$ can be introduced using continuous derivatives and differences of order at most two. In fact, setting

$$
r=r(s):=\max \left\{n \in \mathbb{Z}_{+} ; n<s\right\}
$$

we have the following result, see, e.g., BBI-11, Thm. 2.32].

Proposition 2.10. (a) If $s \in(0, k)$ and noninteger or $s=k$, then

$$
\Lambda^{k, s}=C^{r} \operatorname{Lip}^{s-r} \text {. }
$$

Here $\operatorname{Lip}^{\sigma}:=\Lambda^{1, \sigma}, 0<\sigma \leq 1$.

In particular, $\Lambda^{k, k}=C^{k-1,1}$.

(b) If $s \in(0, k)$ and integer, then

$$
\Lambda^{k, s}=C^{s-1} Z=C^{r} \Lambda^{2,1},
$$

where $Z:=\Lambda^{2,1}$ denotes Zygmund space.

In both cases,

$$
|f|_{C^{k} \Lambda^{k, s}}:=\max _{|\alpha|=r}\left|D^{\alpha} f\right|_{\Lambda^{k, s}} .
$$

The next result goes back to Luzin's classical theorem on approximation of measurable functions on $[0,1]$ by continuous functions in a metric given for $f, g \in[0,1] \rightarrow \mathbb{R}$ by

$$
\rho(f, g):=|\{x \in[0,1] ; f(x) \neq g(x)\}| .
$$

Theorem 2.11. Let $f$ belong to the space $\dot{V}_{p}^{k}$ of smoothness $s \in(0, k]$ and $\varepsilon \in(0,1)$. The following is true.

(a) If $s<k$, then there is a function $f_{\varepsilon} \in \Lambda^{k, s}$ such that

$$
\rho\left(f, f_{\varepsilon}\right)<\varepsilon .
$$

(b) If $s=k$, then there is a function $f_{\varepsilon} \in C^{k}$ such that (2.14) is fulfilled.

Using some facts established in the proof of this theorem we will then study pointwise behaviour of $\dot{V}_{p}^{k}$ functions. Model cases for this are, respectively, Lebesgue's differentiation theorem for $B V$ functions and the Marcinkiewicz theorem [Ma-64] asserting that $f \in B V_{p}$ satisfies the pointwise Lipschitz condition

$$
\varlimsup_{h \rightarrow 0} h^{-\frac{1}{p}}|f(x+h)-f(x)|<\infty
$$

for almost each $x \in[0,1]$.

To formulate the corresponding multivariate results we use the following notions.

Definition 2.12. A function $f \in \ell_{\infty}^{\text {loc }}\left(\mathbb{R}^{d}\right)$ belongs to a one-pointed Lipschitz class $\Lambda^{k, s}\left(x_{0}\right)$ if

$$
\varlimsup_{r \rightarrow 0} r^{-s} \operatorname{Osc}_{k}\left(f ; Q_{r}\left(x_{0}\right)\right)<\infty ;
$$

here $k \in \mathbb{N}, 0<s \leq k$ and $x_{0} \in \stackrel{Q}{ }^{d}$. 
Similarly to the definition of functions from $\Lambda^{k, s}$ we use the notations

$$
\operatorname{Lip}^{s}\left(x_{0}\right):=\Lambda^{1, s}\left(x_{0}\right), \quad Z\left(x_{0}\right):=\Lambda^{2,1}\left(x_{0}\right) .
$$

Further, we define so-called Taylor spaces introduced by Peano Pe-1891 for $d=1$ and studied (and named) by Calderón and Zygmund [CZ-61] for functions from $L_{p}^{\text {loc }}\left(\mathbb{R}^{d}\right)$, $1 \leq p<\infty$.

Definition 2.13. (a) A function $f \in \ell_{\infty}^{\text {loc }}\left(\mathbb{R}^{d}\right)$ belongs to the space $T^{s}\left(x_{0}\right), s>0$, if there is a (Taylor) polynomial $T_{x_{0}}(f)$

$$
T_{x_{0}}(f ; x):=\sum_{|\alpha|<s} \frac{f_{\alpha}\left(x_{0}\right)}{\alpha !}\left(x-x_{0}\right)^{\alpha}
$$

such that

$$
\left|f-T_{x_{0}}(f ; x)\right|=O\left(\left\|x-x_{0}\right\|^{s}\right) \quad \text { as } \quad x \rightarrow x_{0} .
$$

(b) If, moreover, $s$ is integer, then $f \in \ell_{\infty}^{\mathrm{loc}}\left(\mathbb{R}^{d}\right)$ belongs to the space $t^{s}\left(x_{0}\right)$ whenever there exists a polynomial $T_{x_{0}}(f)$ of degree $s$ such that

$$
\left|f-T_{x_{0}}(f ; x)\right|=o\left(\left\|x-x_{0}\right\|^{s}\right) \quad \text { as } \quad x \rightarrow x_{0} .
$$

Remark 2.14. (a) It is readily seen that the polynomials $T_{x_{0}}(f)$ are unique.

(b) If $f \in t^{k}\left(x_{0}\right)$, then the $k$-th homogeneous part of $T_{x_{0}}(f)$

$$
D_{x_{0}}^{k}(f ; x):=\sum_{|\alpha|=k} \frac{f_{\alpha}\left(x_{0}\right)}{\alpha !}\left(x-x_{0}\right)^{\alpha}
$$

is called the Peano $k$-th differential at $x_{0}$. It is the matter of definition to check that the $k$-th differential in the classical sense

$$
\partial_{x_{0}}^{k} f(x):=\sum_{|\alpha|=k} \frac{D^{\alpha} f\left(x_{0}\right)}{\alpha !}\left(x-x_{0}\right)^{\alpha}
$$

coincides with that of Peano for $k=1$. However, this is, in general, incorrect for $k \geq 2$. In particular, Denjoy [De-35] proved that for every nowhere dense closed set $S \subset[0,1]$ and $k \geq 2$ there is a function $f$ whose $k$-th derivative in the classical sense exists only at points of the completion $S^{c}$ of $S$ while the Peano $k$-th derivative exists at all points of $[0,1]$. The converse to this assertion that also proved in [De-35] leads in the multivariate case to the following.

Conjecture. If the Peano $k$-th differential $D_{x}^{k} f, k \geq 2$, exists at every point of $x \in Q^{d}$, then $k$-th differential $\partial_{x}^{k} f$ in the classical sense exists a.e.

(c) Unlike the classical case, the Peano definition can be extended to a much more complicated setting, see, in particular, Br2-15] where this is done for multivariate functions on weak Markov sets including Ahlfors $a$-regular sets in $\mathbb{R}^{d}$ with $d-1<a \leq d$ and fractals of such complicated structure as the von Koch curves, the Cantor sets etc. Such kind of generalization has useful applications in harmonic analysis and singular integral operators theory, see [MZ-36], [CZ-61] and the following up papers. 
Theorem 2.15. Let a function $f$ belong to the space $\dot{V}_{p}^{k}$ of smoothness $s:=\frac{d}{p} \in(0, k]$. Then the following is true for almost each $x_{0} \in Q^{d}$.

(a)

$$
f \in \Lambda^{k, s}\left(x_{0}\right)
$$

(b) If $s=\ell+\lambda<k$, where $\ell \in \mathbb{Z}_{+}$and $0<\lambda \leq 1$, then

$$
f \in T^{s}\left(x_{0}\right)
$$

(c) If $s=k$, then

$$
f \in t^{k}\left(x_{0}\right)
$$

Finally, we present results connecting $\dot{V}_{p}^{k}$ spaces with Lipschitz-Besov spaces over $L_{p}$, $1 \leq p<\infty$. The latter family of spaces is denoted by $\left\{\Lambda_{p q}^{k s}\right\}$, where $k \in \mathbb{N}, 0<s \leq k$ and $1 \leq p, q<\infty$. For its definition we need the following:

Definition 2.16. $(k, p)$-modulus of continuity is a function $L_{p} \times \mathbb{R}_{+} \rightarrow \mathbb{R}_{+}$given for $f \in L_{p}$ and $0<t \leq 1$ by

$$
\omega_{k p}(f ; t):=\sup _{\|h\| \leq t}\left\|\Delta_{h}^{k} f\right\|_{L_{p}\left(Q_{k h}^{d}\right)},
$$

where $\|h\|:=\max _{1 \leq i \leq d}\left|h_{i}\right|$ and

$$
Q_{k h}^{d}:=\left\{x \in Q^{d}: x+k h \in Q^{d}\right\} .
$$

is the domain of the function $x \mapsto\left(\Delta_{h}^{k} f\right)(x)$.

Now the space $\Lambda_{p q}^{k s}$ is defined for $q<\infty$ by the seminorm

$$
|f|_{\Lambda_{p q}^{k s}}:=\left(\int_{0}^{1}\left(\frac{\omega_{k p}(f ; t)}{t^{s}}\right)^{q} \frac{d t}{t}\right)^{\frac{1}{q}}
$$

and for $q=\infty$ by

$$
|f|_{\Lambda_{p \infty}^{k s}}:=\sup _{t>0} \frac{\omega_{k p}(f ; t)}{t^{s}}
$$

For the special case $q=\infty, s=k$ we also use the notation

$$
\operatorname{Lip} p_{p}^{k}:=\Lambda_{p \infty}^{k k} \text {. }
$$

Remark 2.17. (1) We also use the introduced notions for functions on a subcube $Q \subset Q^{d}$. In this case, $f \in L_{p}(Q)$ and the corresponding notions are denoted by $\omega_{k p}(f ; t ; Q), \Lambda_{p q}^{k s}(Q)$ and $\operatorname{Lip} p_{p}^{k}(Q)$.

(2) The space $\Lambda_{p q}^{k s}$ with $k-1 \leq s<k$ coincides with the homogeneous Besov space $\dot{B}_{p q}^{s}$ while the space $\Lambda_{p q}^{k s} / \mathcal{P}_{k-1}^{d}$ up to equivalence of norms coincides with Besov space $B_{p q}^{s}$, see, e.g., [Tr-92, Sec.II.2.3]. 
(3) In the case of one variable, the Hardy-Littlewood theorem [HL-28] asserts (in our notations) that

for $1<p<\infty$ and

$$
\operatorname{Lip}^{\frac{1}{p}} \subset N \dot{V}_{p} \hookrightarrow \operatorname{Lip} p_{p}^{\frac{1}{p}}
$$

$$
\operatorname{Lip}^{1} \subset N \dot{V} \cong \operatorname{Lip}_{1}^{1}
$$

In more details, let $L$ be a linear operator sending a Lebesgue integrable function to its class of equivalence in $L_{1}$. Then the cited result asserts that the trace $\left.L\right|_{N \dot{V}_{p}}$ is a continuous injection into $\operatorname{Lip}_{p}^{1 / p}$ if $1<p<\infty$ and a continuous bijection onto Lip 1 if $p=1$.

Theorem 2.18. (a) If $s:=\frac{d}{p} \in(0, k]$, then the operator $\left.L\right|_{N \dot{V}_{p}^{k}}$ gives the map

$$
N \dot{V}_{p}^{k} \hookrightarrow \Lambda_{p \infty}^{k s}
$$

(Let us recall that if $s=k$ the right-hand side is denoted by Lip $p_{p}^{k}$.)

Moreover, if $0<s<k$, then there is a linear continuous operator right inverse to $\left.L\right|_{N \dot{V}_{p}^{k}}$ that gives the map

$$
\Lambda_{p 1}^{k s} \hookrightarrow N \dot{V}_{p}^{k} .
$$

(b) If $s=k=d$, hence, $p=1$, then $\left.L\right|_{N \dot{V}_{1}^{d}}$ gives the linear isomorphism

$$
N \dot{V}_{1}^{d} \cong \operatorname{Lip}_{1}^{d} \text {. }
$$

Let us note that embedding (2.24) and isomorphism (2.26) give the above mentioned Hardy-Littlewood results while embedding (2.25) is apparently new even for this case.

Remark 2.19. The left-side Hardy-Littlewood embeddings also can be extended to the multivariate case, we have

$$
\Lambda^{k, s} \subset \dot{V}_{p}^{k}, \quad s:=\frac{d}{p} \in(0, k] .
$$

In fact, by definition, see (2.8), (2.9),

$$
\operatorname{osc}_{k}(f ; Q) \leq|Q|^{\frac{s}{d}}|f|_{\Lambda^{k, s}}, \quad Q \subset Q^{d},
$$

while by Theorem $2.8|f|_{V_{p}^{k}}$ is majorated by

$$
\sup _{\pi}\left\{\sum_{Q \in \pi} \operatorname{osc}_{k}(f ; Q)^{p}\right\}^{\frac{1}{p}} \leq \sup _{\pi}\left\{\sum_{Q \in \pi}|Q|^{\frac{s p}{d}}\right\}^{\frac{1}{p}}|f|_{\Lambda^{k, s}}=|f|_{\Lambda^{k, s}} .
$$

As a consequence of Theorem 2.18 (c) we present the multivariate generalization of the classical Lebesgue-Vitali theorem on absolutely continuous functions formulated as property (d) of the space $\dot{V}:=\dot{V}_{1}^{d}$ in Section 1.1. Let us recall that $A C$ denotes the space of functions $f \in \dot{V}$ satisfying the condition

$$
\lim _{\varepsilon \rightarrow 0} \sup _{|\pi| \leq \varepsilon} \sum_{Q \in \pi} \operatorname{osc}_{d}(f ; Q)=0
$$


here $|\pi|:=\sum_{Q \in \pi}|Q|$ and $\operatorname{osc}_{d}$ can be replaced by $E_{d}$, see Theorems 2.8, 6.1.

Corollary 2.20. The map sending a function from $\dot{V}$ to its equivalence class in $L_{1}$ gives rise to the isomorphism

$$
A C \cong \dot{W}_{1}^{d} .
$$

Moreover, there are a continuous linear integral operator $J: L_{1}\left(Q^{d} ; \mathbb{R}^{N}\right) \rightarrow C$ and a linear projection $P: L_{1} \rightarrow \mathcal{P}_{d-1}^{d}$ such that for every $f \in A C$

$$
f=P f+J\left(\nabla^{d} f\right)
$$

here $\nabla^{d} f:=\left(D^{\alpha} f\right)_{|\alpha|=d}$ and $N:=\operatorname{card}\left\{\alpha \in \mathbb{Z}_{+}^{d}:|\alpha|=d\right\}$.

2.3. Duality Theorems. Now we introduce a predual to $V_{p}^{k}$ space denoted by $U_{p}^{k}$ and describe its properties.

The space under consideration is constructed by the following building blocks called $(k, p)$-atoms.

Definition 2.21. A function $a \in \ell_{1}\left(=\ell_{1}\left(Q^{d}\right)\right)$ is said to be $a(k, p)$-atom if it is such that

(a) a is supported by a subcube $Q \subset Q^{d}$;

(b) $\|a\|_{1} \leq 1$;

(c) $\sum_{x \in Q^{d}} x^{\alpha} a(x)=0$ for all $|\alpha| \leq k-1$.

The subject of the definition is denoted by $a_{Q}$.

Definition $2.22\left((k, p)\right.$-chain). A function $b \in \ell_{1}$ is said to be a $(k, p)$-chain subordinate to a packing $\pi \in \Pi$ if $b$ belongs to the linear hull of the family $\left\{a_{Q}\right\}_{Q \in \pi}$.

The subject of the definition is denoted by $b_{\pi}$.

Moreover, we set

$$
\left[b_{\pi}\right]_{p^{\prime}}:=\left\|\left\{c_{Q}\right\}_{Q \in \pi}\right\|_{p^{\prime}}:=\left(\sum_{Q \in \pi}\left|c_{Q}\right|^{p^{\prime}}\right)^{\frac{1}{p^{\prime}}}
$$

whenever

$$
b_{\pi}=\sum_{Q \in \pi} c_{Q} a_{Q}, \quad\left\{c_{Q}\right\}_{Q \in \pi} \subset \mathbb{R} .
$$

(As usual, $\frac{1}{p}+\frac{1}{p^{\prime}}=1$.)

Further, we define a dense subspace of the required predual space denoted by $\left(U_{p}^{k}\right)^{0}$ by setting

$$
\left(U_{p}^{k}\right)^{0}:=\operatorname{linhull}\left(\mathcal{A}_{p}^{k}\right),
$$

where $\mathcal{A}_{p}^{k}$ is the family of all $(k, p)$-atoms. 
Equivalently,

$$
\left(U_{p}^{k}\right)^{0}:=\operatorname{linhull}\left(\mathcal{B}_{p}^{k}\right),
$$

where $\mathcal{B}_{p}^{k}$ is the family of all $(k, p)$-chains.

Moreover, we equip $\left(U_{p}^{k}\right)^{0}$ with a seminorm $f \mapsto\|f\|_{U_{p}^{k}}$ given for $f \in\left(U_{p}^{k}\right)^{0}$ by

$$
\|f\|_{U_{p}^{k}}:=\inf \sum_{\pi}\left[b_{\pi}\right]_{p^{\prime}}
$$

where infimum is taken over all presentations

$$
f=\sum_{\pi} b_{\pi} .
$$

Definition 2.23. The space $U_{p}^{k}$ is completion of the space $\left(\left(U_{p}^{k}\right)^{0},\|\cdot\|_{U_{p}^{k}}\right)$.

Now we describe the properties of the space $U_{p}^{k}$.

In the formulation of the result, we use a space $U_{p \infty}^{k}$ defined by the very same construction as $U_{p}^{k}$ but with $\ell_{1}$ replaced by $L_{1}$. Hence, in this case $a_{Q}$ is an $L_{1}$ function supported by a subcube $Q$ orthogonal to $\mathcal{P}_{k-1}^{d}$ and such that $\int_{Q^{d}}\left|a_{Q}\right| d x \leq 1$.

It follows from [BB-18, Th. 2.6] that

$$
\left(U_{p \infty}^{k}\right)^{*} \equiv V_{p \infty}^{k}
$$

Theorem 2.24. (a) $U_{p}^{k}$ is a Banach space.

(b) $B\left(U_{p}^{k}\right)$ is the closure of the symmetric convex hull of the set $\left\{b_{\pi} \in\left(U_{p}^{k}\right)^{0}:\left[b_{\pi}\right]_{p^{\prime}} \leq 1\right\}$.

(c) The space $U_{p}^{k}$ is nonseparable and contains a separable subspace, say, $\hat{U}_{p}^{k}$, such that

$$
\hat{U}_{p}^{k} \equiv U_{p \infty}^{k} \quad \text { and } \quad\left(U_{p}^{k} / \hat{U}_{p}^{k}\right)^{*} \cong \ell_{p} .
$$

Finally, we present the duality theorem for the Banach space $V_{p}^{k}\left(:=\dot{V}_{p}^{k} / \mathcal{P}_{k-1}^{d}\right.$, see $(1.22)$ ).

Theorem 2.25. It is true that

$$
\left(U_{p}^{k}\right)^{*} \equiv V_{p}^{k}
$$

In more details, every linear functional $f \in\left(U_{p}^{k}\right)^{*}$ is assigned at $u \in\left(U_{p}^{k}\right)^{0}$ by

$$
\langle f, u\rangle:=\sum_{x \in Q^{d}} v_{f}(x) u(x),
$$

where $v_{f}$ is a uniquely defined function of $\dot{V}_{p}^{k}$ linearly depending on $f$.

Moreover, it is true that

$$
\|f\|_{\left(U_{p}^{k}\right)^{*}}=\left|v_{f}\right|_{V_{p}^{k}} .
$$

\footnotetext{
${ }^{4}$ In fact, norm, see Theorem 2.24(a) below.
} 
2.4. Two Stars Theorems. Now we present duality results connecting the space $\mathrm{V}_{p}^{k}$ with the spaces $V_{p}^{k}$ and $U_{p}^{k}$. In their proofs an essential role plays an extension of the space $U_{p}^{k}$ denoted by $\widetilde{U}_{p}^{k}$. In its definition, we use the Banach space $M$ of finite signed Borel measures on $Q^{d}$ equipped with a norm given for $\mu \in M$ by

$$
\|\mu\|_{M}:=\underset{Q^{d}}{\operatorname{var}} \mu \text {. }
$$

We define $\widetilde{U}_{p}^{k}$ as a direct analog of the space $U_{p}^{k}$ using as building blocks atoms $\left\{\mu_{Q}\right\}_{Q \subset Q^{d}} \subset$ $M$ satisfying the conditions

(i) $\operatorname{supp} \mu \subset Q$;

(ii) $\|\mu\|_{M} \leq 1$;

(iii) $\int_{Q^{d}} x^{\alpha} d \mu=0$ for all $|\alpha| \leq k-1$.

The corresponding linear hull of the set $\left\{\mu_{Q}\right\}_{Q \subset Q^{d}}$ is denoted by $\left(\widetilde{U}_{p}^{k}\right)^{0}$ and by $\widetilde{U}_{p}^{k}$ is denoted its completion with respect to a seminorm defined similarly to that for $\left(U_{p}^{k}\right)^{0}$.

Repeating line-by-line the proof of Theorem 2.24 (a) we will show that the space $\widetilde{U}_{p}^{k}$ is Banach.

Since every chain $b \in\left(U_{p}^{k}\right)^{0}$ can be identified with a discrete measure $\mu_{b} \in M$ with $\mu_{b}(\{x\}):=b(x), x \in Q^{d}$, the space $\left(U_{p}^{k}\right)^{0}$ is embedded (as a vector space) in $\left(\widetilde{U}_{p}^{k}\right)^{0}$.

Theorem 2.26. (a) The map $\left(U_{p}^{k}\right)^{0} \rightarrow\left(\widetilde{U}_{p}^{k}\right)^{0}, b \mapsto \mu_{b}$, extends to an isometric embedding of $U_{p}^{k}$ into $\widetilde{U}_{p}^{k}$ denoted by $\mathcal{I}$.

(b) There exists a linear continuous surjection of $\widetilde{U}_{p}^{k}$ onto $U_{p}^{k}$ denoted by $\mathcal{E}$ such that

$$
\operatorname{ker}(\mathcal{E}) \cap\left(\widetilde{U}_{p}^{k}\right)^{0}=(0) \quad \text { and } \quad \mathcal{E} \circ \mathcal{I}=\mathrm{id} .
$$

The result allows to identify $\left(\widetilde{U}_{p}^{k}\right)^{0} \subset M$ with a dense subspace of $U_{p}^{k}$ containing $\left(U_{p}^{k}\right)^{0}$ and to apply the Riesz representation theorem in the forthcoming proofs.

Now we formulate a result giving another presentation of the predual space $U_{p}^{k}$ and derive from here the mentioned two stars theorem.

Theorem 2.27. If the space $V_{p}^{k}$ has smoothness $s<k$ and $p>1$, then

$$
\left(\mathrm{V}_{p}^{k}\right)^{*} \cong U_{p}^{k}
$$

In more details, every linear continuous functional on $\mathrm{V}_{p}^{k}$ is of the form

$$
T_{u}(f)=f(u), \quad f \in \mathrm{v}_{p}^{k},
$$

where $u \in U_{p}^{k}$ is a uniquely defined element such that

$$
\left\|T_{u}\right\|_{\left(\mathrm{v}_{p}^{k}\right)^{*}} \approx\|u\|_{U_{p}^{k}}
$$

with the constants of equivalence independent of $u$.

As a direct consequence we have the following 
Corollary 2.28 (Two stars theorem). If $V_{p}^{k}$ satisfies the conditions $s<k$ and $p>1$, then

$$
\left(\mathrm{v}_{p}^{k}\right)^{* *} \cong V_{p}^{k}
$$

\section{Proofs of Theorem 2.1 and Corollary 2.2}

3.1. Auxiliary Results. Here we prove some auxiliary results that are interesting in their own right.

Lemma 3.1. Let $\left\{Q_{i}\right\}_{i \in \mathbb{N}}$ be the family of nonoverlapping cubes in $Q^{d}$. Then for each $f \in \dot{V}_{p}^{k}$ the limit $\lim _{i \rightarrow \infty} E_{k}\left(f ; Q_{i}\right)$ exists and equals 0 .

Proof. By the definition of the seminorm on $\dot{V}_{p}^{k}$, see (1.20), we have

$$
\sum_{i=1}^{\infty} E_{k}\left(f ; Q_{i}\right)^{p} \leq|f|_{V_{p}^{k}}^{p}<\infty .
$$

Since $1 \leq p<\infty$, this implies the required statement.

Further, let $\mathcal{Q}_{x}$ denote the family of subcubes of $Q^{d}$ containing a point $x \in Q^{d}$. We set for a function $f \in \dot{V}_{p}^{k}$,

$$
f_{k}(x):=\varlimsup_{\mathcal{Q}_{x} \ni Q \rightarrow\{x\}} E_{k}(f ; Q) ;
$$

here the convergence is in the Hausdorff metric on subsets of $\mathbb{R}^{d}$.

Clearly, $0 \leq f_{k}(x) \leq|f|_{V_{p}^{k}}, x \in Q^{d}$. In fact, the following result holds.

Lemma 3.2. The function $f_{k} \in \ell_{p}$ and satisfies the inequality $\left\|f_{k}\right\|_{p} \leq|f|_{V_{p}^{k}}$.

Proof. Assume that $c$ is a non-isolated point of range $\left(f_{k}\right):=f_{k}\left(Q^{d}\right)$. Then there exists a sequence of distinct points $\left\{x_{i}\right\}_{i \in \mathbb{N}} \subset Q^{d}$ such that $\lim _{i \rightarrow \infty} f_{k}\left(x_{i}\right)=c$. In turn, by the definition of $f_{k}$, there is a family of nonoverlapping cubes $Q_{i} \subset \mathcal{Q}_{x_{i}}$ such that $E_{k}\left(f ; Q_{i}\right) \geq$ $\frac{i-1}{i} f_{k}\left(x_{i}\right), i \in \mathbb{N}$. Hence, due to Lemma 3.1.

$$
0 \leq c=\lim _{i \rightarrow \infty} f_{k}\left(x_{i}\right) \leq \lim _{i \rightarrow \infty} E_{k}\left(f ; Q_{i}\right)=0 .
$$

Hence, the set range $\left(f_{k}\right) \backslash\{0\}$ consists of isolated points only and is at most countable.

Next, if $c \in$ range $\left(f_{k}\right) \backslash\{0\}$, then the set $f_{k}^{-1}(c) \subset Q^{d}$ is finite. (In fact, otherwise there is a family of nonoverlapping cubes $\left\{Q_{i}\right\}_{i \in \mathbb{N}}$ such that $\lim _{i \rightarrow \infty} E_{k}\left(f ; Q_{i}\right)=c \neq 0$ which contradicts Lemma 3.1).

These, in turn, imply that $\operatorname{supp} f_{k}:=f_{k}^{-1}\left(\operatorname{range}\left(f_{k}\right) \backslash\{0\}\right)$ is at most countable. Moreover, $\| f_{k}$; supp $f_{k} \|_{p} \leq|f|_{V_{p}^{k}}$, by the definitions of $f_{k}$ and the seminorm on $\dot{V}_{p}^{k}$.

Proposition 3.3. Let $x \in Q^{d}$. Then $f_{k}(x)=0$ iff $f$ is continuous at $x$. 
Proof. Suppose that $x \in Q^{d}$ is such that $f_{k}(x)=0$. We prove that $f$ is continuous at $x$.

Since $f \in \ell_{\infty}$, each infinite subset of range $(f)$ has a limit point. Hence, we should prove that if $\left\{x_{i}\right\}_{i \in \mathbb{N}} \subset Q^{d}$ is a sequence converging to $x$ and the sequence $\left\{f\left(x_{i}\right)\right\}_{i \in \mathbb{N}}$ converges to some $c \in \mathbb{R}$, then $c=f(x)$.

To this end, passing to a subsequence of $\left\{x_{i}\right\}_{i \in \mathbb{N}}$, if necessary, without loss of generality we can assume that

$$
\lim _{i \rightarrow \infty} \frac{\left\|x_{i+1}-x\right\|_{\ell_{\infty}^{d}}}{\left\|x_{i+1}-x_{i}\right\|_{\ell_{\infty}^{d}}}=0
$$

Since the projections of the interval $\left[x, x_{i}\right] \subset Q^{d}$ onto the coordinate axes have lengths bounded from above by $\left\|x-x_{i}\right\|_{\ell_{\infty}^{d}}(\leq 1)$, there exists a cube $Q_{i} \in \mathcal{Q}_{x}$ of sidelength $\left\|x-x_{i}\right\|_{\ell_{\infty}^{d}}$ containing $\left[x, x_{i}\right]$.

In particular, the sequence $\left\{Q_{i}\right\}_{i \in \mathbb{N}}$ converges to $\{x\}$ in the Hausdorff metric.

Moreover, sets $Q_{i} \cup Q_{i+1}$ are connected so that their projections on the coordinate axes are compact subintervals of $[0,1]$ of lengths $\leq\left\|x-x_{i}\right\|_{\ell_{\infty}^{d}}+\left\|x-x_{i+1}\right\|_{\ell_{\infty}^{d}}$.

Hence, there exists a cube $Q_{i, i+1} \in \mathcal{Q}_{x}$ of sidelength $\leq\left\|x-x_{i}\right\|_{\ell_{\infty}^{d}}+\left\|x-x_{i+1}\right\|_{\ell_{\infty}^{d}}$ containing $Q_{i} \cup Q_{i+1}$. In particular, $Q_{i, i+1} \rightarrow\{x\}$ in the Hausdorff metric as $x \rightarrow \infty$.

Now let $m_{i, i+1} \in \mathcal{P}_{k-1}^{d}, i \in \mathbb{N}$, be such that

$$
E_{k}\left(f ; Q_{i, i+1}\right)=\left\|f-m_{i, i+1} ; Q_{i, i+1}\right\|_{\infty} .
$$

Since $\left\|m_{i, i+1} ; Q_{i, i+1}\right\|_{\infty} \leq\|f\|_{\infty}$ and $Q_{i, i+1}$ contains the interval $\left[x_{i}, x_{i+1}\right]$, the mean-value theorem and the A. Markov inequality for derivatives of polynomials give for a positive constant $c:=c(k, d)$

$$
\begin{aligned}
\left|f(x)-f\left(x_{i+1}\right)\right| & \leq\left|f(x)-m_{i, i+1}(x)\right|+\left|f\left(x_{i+1}\right)-m_{i, i+1}\left(x_{i+1}\right)\right| \\
& +\left|m_{i, i+1}(x)-m_{i, i+1}\left(x_{i+1}\right)\right| \leq 2 E_{k}\left(f ; Q_{i, i+1}\right)+\max _{Q_{i, i+1}}\left\|\nabla m_{i, i+1}\right\|_{\ell_{\infty}^{d}} \\
& \leq 2 E_{k}\left(f ; Q_{i, i+1}\right)+\frac{c}{\operatorname{diam}\left(Q_{i, i+1}\right)}\|f\|_{\infty}\left\|x-x_{i+1}\right\|_{\ell_{\infty}^{d}} \\
& \leq 2 E_{k}\left(f ; Q_{i, i+1}\right)+\frac{c\left\|x-x_{i+1}\right\|_{\ell_{\infty}^{d}}}{\left\|x_{i}-x_{i+1}\right\|_{\ell_{\infty}^{d}}}\|f\|_{\infty} .
\end{aligned}
$$

Further, since $f_{k}(x)=0$, condition (3.1) implies that

$$
\lim _{\mathcal{Q}_{x} \ni Q \rightarrow\{x\}} E_{k}(f ; Q)=0 .
$$

From here and (3.2) we obtain that the right-hand side of (3.3) tends to 0 as $i$ tends to $\infty$. Thus, $c=\lim _{i \rightarrow \infty} f\left(x_{i+1}\right)=f(x)$, as required, i.e., $f$ is continuous at $x$.

Conversely, suppose that $f \in \dot{V}_{p}^{k}$ is continuous at $x \in Q^{d}$. Then

$$
0 \leq f_{k}(x):=\varlimsup_{\mathcal{Q}_{x} \ni Q \rightarrow\{x\}} E_{k}(f ; Q) \leq \varlimsup_{\mathcal{Q}_{x} \ni Q \rightarrow\{x\}} E_{1}(f ; Q) \leq \varlimsup_{\mathcal{Q}_{x} \ni Q \rightarrow\{x\}}\|f-f(x) ; Q\|_{\infty}=0 .
$$


Hence, $f_{k}(x)=0$. This completes the proof of the proposition.

Proposition 3.4. Let $\ell$ be a line intersecting $Q^{d}$ by a nontrivial interval $I$. Then for each $f \in \dot{V}_{p}^{k}$ its restriction to $I$ belongs to the space $\dot{V}_{p}^{k}(I)$.

Proof. We need the following geometric result.

Lemma 3.5. Let $\left\{I_{j}\right\}_{1 \leq j \leq s}$ be a family of nonoverlapping nontrivial closed intervals in $I$. There exists a family of nonoverlapping cubes $\left\{Q_{j}\right\}_{1 \leq j \leq s}$ in $Q^{d}$ such that

$$
\left|Q_{j}\right|^{\frac{1}{d}}=\ell_{\infty}\left(I_{j}\right) \quad \text { and } \quad Q_{j} \cap I=I_{j}, \quad 1 \leq j \leq s
$$

here $\ell_{\infty}([x, y]):=\max _{1 \leq i \leq d}\left\{\left|x_{i}-y_{i}\right|\right\}$ is the $\ell_{\infty}^{d}$-diameter of $[x, y] \subset \mathbb{R}^{d}$.

Proof. Let $\alpha_{i}$ be the angle between a vector parallel to $I$ and the coordinate vector $e_{i}$, $1 \leq i \leq d$. Renumerating the coordinates axes, if necessary, without loss of generality we assume that the sequence $\left\{\left|\cos \alpha_{i}\right|\right\}_{1 \leq i \leq d}$ is nonincreasing. Then the lengths of the orthogonal projections of every $I_{j}$ on the coordinate axes $x_{i}, 1 \leq i \leq d$, also form a nonincreasing sequence.

Using this we proceed by induction on $d$ starting from the trivial case $d=1$ and assuming that the result holds for all dimensions $\leq d-1$ with $d \geq 2$.

To prove the lemma for $d \geq 2$ we use the map $\pi_{d}: x \mapsto\left(x_{1}, \ldots, x_{d-1}, 0\right)$ sending $Q^{d}$ onto $Q^{d-1}:=\left\{x \in Q^{d}: x_{d}=0\right\}$. Then $I^{\prime}:=\pi_{d}(I)$ is a closed interval in the cube $Q^{d-1}$ and $I_{j}^{\prime}:=\pi_{d}\left(I_{j}\right), 1 \leq j \leq s$, is a family of nonoverlapping closed intervals in $I^{\prime}$. Moreover, $I^{\prime}$ is nontrivial (hence, every $I_{j}^{\prime}$ is) since otherwise $\left|\cos \alpha_{d}\right|=0$ and, hence, by our assumption all $\left|\cos \alpha_{j}\right|=0$ contradicting nontriviality of $I$.

Now by the induction hypothesis there is a family of nonoverlapping cubes $Q_{j}^{\prime} \subset Q^{d-1}$, $1 \leq j \leq s$, satisfying condition (3.4) for the family $\left\{I_{j}^{\prime}\right\}_{1 \leq j \leq s}$.

Next, preimages of $Q_{j}^{\prime}$ with respect to the map $\left.\pi_{d}\right|_{Q^{d}}$ form the family of nonoverlapping rectangular parallelotopes, say $\tilde{Q}_{j}$, with bases $Q_{j}^{\prime}$ and heights being the unit intervals parallel to the $x_{d^{-}}$axis; moreover, $I_{j}=\ell \cap \tilde{Q}_{j}, 1 \leq j \leq s$.

Let $I_{j, d}$ be the image of $I_{j}$ under the orthogonal projection onto the $x_{d}$ axis. Then $I_{j, d}$ is a (possibly trivial) interval of length $\leq \ell_{\infty}\left(I_{j}\right) \leq 1$ in the unit interval in this axes. In particular, there is a subinterval $I_{j, d}^{\prime}$ in the unit interval of length $\ell_{\infty}\left(I_{j}\right)$ containing $I_{j, d}$.

The intersection of the preimage of this interval under the projection onto $x_{d}$ with $\tilde{Q}_{j}$ is clearly a cube $Q_{j}$ of sidelength $\ell_{\infty}\left(I_{j}\right)$ satisfying the required statement.

Finally, using the result and notations of Lemma 3.5, for each $f \in \dot{V}_{p}^{k}$ we have

$$
E_{k}\left(f ; I_{j}\right) \leq E_{k}\left(f ; Q_{j}\right), \quad 1 \leq j \leq s
$$

In particular,

$$
\left(\sum_{j=1}^{s} E_{k}\left(f ; I_{j}\right)^{p}\right)^{\frac{1}{p}} \leq\left(\sum_{j=1}^{s} E_{k}\left(f ; Q_{j}\right)^{p}\right)^{\frac{1}{p}} \leq|f|_{V_{p}^{k}} .
$$


Taking in the left-hand side supremum over all packings $\left\{I_{j}\right\} \subset I$ we obtain that $\left.f\right|_{I} \in$ $\dot{V}_{p}^{k}(I)$ and $\left.|f|_{I}\right|_{V_{p}^{k}(I)} \leq|f|_{V_{p}^{k}}$.

The proof of the proposition is complete.

\subsection{Proof of Theorem 2.1.}

Proof. (a) Suppose that $d=1$ and $f \in \dot{V}_{p}^{k}([0,1])$. We should prove that $f$ has one-sided limits at each point $x \in[0,1]$.

Since $f \in \ell_{\infty}$, each infinite subset of range $(f)$ has a limit point. Hence, we should prove that if $\left\{x_{j, i}\right\}_{i \in \mathbb{N}} \subset[0,1], j=1,2$, are sequences converging to $x$ from the same side such that sequences $\left\{f\left(x_{j, i}\right)\right\}_{i \in \mathbb{N}}$ converge to some $c_{j} \in \mathbb{R}, j=1,2$, then $c_{1}=c_{2}$.

To this end, passing to subsequences of $\left\{x_{j, i}\right\}_{i \in \mathbb{N}}, j=1,2$, if necessary, without loss of generality we may assume that

$$
x_{1, i+1}<x_{2, i+1}<x_{1, i}<x_{2, i} \quad \text { for all } i \in \mathbb{N} \quad \text { and } \quad \lim _{i \rightarrow \infty} \frac{\left|x_{2, i+1}-x_{1, i+1}\right|}{\left|x_{2, i+1}-x_{1, i}\right|}=0 ;
$$

here all intervals $I_{i}:=\left[x_{1, i}, x_{2, i}\right] \subset[0,1], i \in \mathbb{N}$, belong to the same connected component of the set $\mathbb{R} \backslash\{x\}$.

By definition, $\left\{I_{i}\right\}_{i \in \mathbb{N}} \subset[0,1]$ is a family of nonoverlapping intervals converging to $\{x\}$ in the Hausdorff metric. The second condition (3.5) implies that there exists a family of nonoverlapping intervals $\left\{I_{i}^{\prime}\right\}_{i \in \mathbb{N}} \subset[0,1]$ such that

$$
I_{i} \subset I_{i}^{\prime} \quad \text { for all } \quad i \in \mathbb{N}, \quad \lim _{i \rightarrow \infty} \frac{\left|I_{i}\right|}{\left|I_{i}^{\prime}\right|}=0, \quad \text { and } \quad \lim _{i \rightarrow \infty} I_{i}^{\prime}=\{x\}
$$

in the Hausdorff metric.

In particular, due to Lemma 3.1 ,

$$
\lim _{i \rightarrow \infty} E_{k}\left(f ; I_{i}^{\prime}\right)=0 .
$$

Let $m_{i} \in \mathcal{P}_{k-1}^{1}, i \in \mathbb{N}$, be such that

$$
E_{k}\left(f ; I_{i}^{\prime}\right)=\left\|f-m_{i} ; I_{i}^{\prime}\right\|_{\infty} .
$$

Since $\left\|m_{i} ; I_{i}^{\prime}\right\|_{\infty} \leq\|f\|_{\infty}$ and $I_{i}^{\prime} \supset I_{i}$, the mean-value theorem and the A. Markov inequality for derivatives of polynomials give

$$
\begin{aligned}
\left|f\left(x_{2, i}\right)-f\left(x_{1, i}\right)\right| & \leq\left|f\left(x_{2, i}\right)-m_{i}\left(x_{2, i}\right)\right|+\left|f\left(x_{1, i}\right)-m_{i}\left(x_{1, i}\right)\right|+\left|m_{i}\left(x_{2, i}\right)-m_{i}\left(x_{1, i}\right)\right| \\
& \leq 2 E_{k}\left(f ; I_{i}^{\prime}\right)+\frac{2(k-1)^{2}}{\left|I_{i}^{\prime}\right|}\|f\|_{\infty}\left|I_{i}\right| .
\end{aligned}
$$

Since the right-hand side of the inequality tends to 0 as $i \rightarrow \infty$, see (3.6), (3.7),

$$
c_{1}=\lim _{i \rightarrow \infty} f\left(x_{1, i}\right)=\lim _{i \rightarrow \infty} f\left(x_{2, i}\right)=c_{2} .
$$

This completes the proof of part (a) of the theorem.

(b) Now suppose that $f \in \dot{V}_{p}^{k}\left(Q^{d}\right)$ with $d \geq 2$. We should prove that $f$ has limits at each point $x \in Q^{d}$. 
First, due to Proposition 3.4, for each line $\ell$ through $x$ such that $I:=\ell \cap Q^{d} \neq\{x\}$ the restriction $\left.f\right|_{I}$ belongs to $\dot{V}_{p}^{k}(I)$. In particular, according to (a) $\left.f\right|_{I}$ has one sided limits at $x$. This implies that $f$ has radial limits at $x$.

Next, we show that all these limits coincide.

Let $H_{x}$ be a hyperplane through $x$ parallel to a coordinate hyperplane and $x_{j}$ be the coordinate axis orthogonal to $H_{x}$. Then $\mathbb{R}^{d} \backslash H_{x}$ is the union of two nonintersecting open half spaces $S_{1}, S_{2}$ such that at least one of them, say $S_{1}$, intersects $Q^{d}$.

Lemma 3.6. Let $y_{1}, y_{2} \in S_{1} \cap Q^{d}$ be distinct. Then

$$
\lim _{\left(x, y_{1}\right] \ni z \rightarrow x} f(z)=\lim _{\left(x, y_{2}\right] \ni z \rightarrow x} f(z) .
$$

Proof. By our assumption, the orthogonal projection onto the $x_{j}$ axis $p_{j}$ maps lines $\left(x, y_{i}\right)$, $i=1,2$, bijectively onto $x_{j}$. Let

$$
I:=p_{j}\left(\left(x, y_{1}\right]\right) \cap p_{j}\left(\left(x, y_{2}\right]\right)
$$

be the half open interval with the endpoint $p_{j}(x)$. We choose a monotonic (with respect to the natural order on $x_{j}$ ) sequence $\left\{z_{n}\right\}_{n \in \mathbb{N}} \subset I$ converging to $p_{j}(x)$ and set

$$
y_{n, i}:=p_{j}^{-1}\left(z_{n}\right) \cap\left(x, y_{i}\right), \quad i=1,2 .
$$

Passing to a subsequence of $\left\{z_{n}\right\}_{n \in \mathbb{N}}$, if necessary, without loss of generality we assume that

$$
\lim _{n \rightarrow \infty} \frac{\max _{i=1,2}\left\|y_{n+1, i}-x\right\|_{\ell_{\infty}^{d}}}{\left\|z_{n+1}-z_{n}\right\|_{\ell_{\infty}^{d}}}=0 .
$$

Next, let $P_{n}:=p_{j}^{-1}\left(\left[z_{n}, z_{n+1}\right]\right) \cap Q^{d}$ be the rectangular parallelotope between hyperplanes $p_{j}^{-1}\left(z_{n+1}\right)$ and $p_{j}^{-1}\left(z_{n}\right)$ inside $Q^{d}$. Then $P_{n}$ contains points $y_{n+1, i}, i=1,2$, and the smallest length of projections of $P_{n}$ onto coordinate axes is $\left\|z_{n+1}-z_{n}\right\|_{\ell_{\infty}^{d}}$. Moreover, due to (3.9),

$$
\left\|y_{n+1,2}-y_{n+1,1}\right\|_{\ell_{\infty}^{d}} \leq 2 \max _{i}\left\|y_{n+1, i}-x\right\|_{\ell_{\infty}^{d}}=\lambda_{n} \cdot\left\|z_{n+1}-z_{n}\right\|_{\ell_{\infty}^{d}},
$$

where the sequence $\left\{\lambda_{n}\right\}_{n \in \mathbb{N}}$ converges to 0 .

These imply that for each $n$ such that $\lambda_{n}<1$ there exists a cube $Q_{n} \subset P_{n}$ of sidelength $\left\|z_{n+1}-z_{n}\right\|_{\ell_{\infty}^{d}}$ containing points $y_{n+1, i}, i=1,2$.

By the definition, $\left\{Q_{n}\right\}_{n \in \mathbb{N}}$ is a sequence of nonoverlapping cubes converging to $\{x\}$ in the Hausdorff metric. Hence, according to Lemma 3.1

$$
\lim _{n \rightarrow \infty} E_{k}\left(f ; Q_{n}\right)=0 \text {. }
$$

Then as in (3.3) using the mean-value theorem and the Markov inequality for derivatives of polynomials on $Q_{n}$, and after that using (3.9), (3.10), we obtain for some $c=c(k, d)$

$$
\varlimsup_{n \rightarrow \infty}\left|f\left(y_{n+1,1}\right)-f\left(y_{n+1,2}\right)\right| \leq \varlimsup_{n \rightarrow \infty}\left(2 E\left(f ; Q_{n}\right)+\frac{c\left\|y_{n+1,2}-y_{n+1,1}\right\|_{\ell_{\infty}^{d}}}{\left\|z_{n+1}-z_{n}\right\|_{\ell_{\infty}^{d}}}\|f\|_{\infty}\right)=0 .
$$

This implies (3.8). 
Using Lemma 3.6 we obtain the following:

Lemma 3.7. If $d \geq 2$, then all radial limits of $f \in \dot{V}_{p}^{k}\left(Q^{d}\right)$ at a point $x \in Q^{d}$ coincide.

Proof. First, observe that $Q^{d} \backslash\{x\}$ is covered by the union of open sets $\mathbb{R}^{d} \backslash H_{x}^{i}, 1 \leq i \leq d$, where $H_{x}^{i}$ is a hyperplane through $x$ orthogonal to the $x_{i}$ axes; this is true because

$$
\bigcap_{1 \leq i \leq d} H_{x}^{i}=\{x\}
$$

Let $\left\{S_{j}\right\}_{1 \leq j \leq k}, d \leq k \leq 2 d$, be the family of all connected components (open half spaces) of the sets $\mathbb{R}^{d} \backslash H_{x}^{i}, 1 \leq i \leq d$, intersecting $Q^{d}$. According to Lemma 3.6, $f$ has the same radial limit at $x$ along each ray in $S_{j}$ emanating from $x$ denoted by $L_{j}$.

Clearly,

$$
Q^{d} \backslash\{x\} \subset \bigcup_{1 \leq j \leq k} S_{j}
$$

In particular, for a path $\gamma:[0,1] \rightarrow Q^{d} \backslash\{x\}$ there is a subset $J \subset\{1, \ldots, k\}$ such that

$$
\gamma([0,1]) \subset \bigcup_{j \in J} S_{j} \quad \text { and } \quad S_{j} \cap \gamma([0,1]) \neq \emptyset, \quad j \in J .
$$

Thus, the function

$$
F_{f}(t):=\lim _{(x, \gamma(t)] \ni z \rightarrow x} f(z), \quad t \in[0,1],
$$

attains value $L_{j}$ on the open set $\gamma^{-1}\left(S_{j}\right), j \in J$. Since these open sets cover the connected interval $[0,1]$, the function $F_{f}$ must be constant.

Hence, $f$ has the same radial limits at $x$ along the rays emanating from $x$ passing through $\gamma(0)$ and $\gamma(1)$.

Finally, since $Q^{d} \backslash\{x\}$ is path connected, every two its points are joined by a path. Together with the previous argument this completes the proof of the lemma.

To proceed with the proof of the theorem, we require the following geometric result.

Proposition 3.8. Let $\left\{R_{n}\right\}_{n \in \mathbb{N}} \subset \mathbb{R}^{d}$ be a sequence of rays with the origin at $x \in Q^{d}$ converging to a ray $R$. If $R_{n} \cap Q^{d} \backslash\{x\} \neq \emptyset$ for all $n$, then $R \cap Q^{d} \backslash\{x\} \neq \emptyset$ as well.

Here convergence is defined by the points of intersection of the rays with the unit sphere centered at $x$.

Proof. We apply induction on dimension.

For $d=1$ the result trivially holds. Assuming that it is valid for all dimensions $\leq d-1$, $d \geq 2$, we prove it for $d$.

To this end, consider intervals $I_{n}:=\left[x, x_{n}\right]:=R_{n} \cap Q^{d}, n \in \mathbb{N}$. By the assumption of the lemma $\left\|x_{n}-x\right\|_{\ell_{\infty}^{d}}>0$. Passing to a subsequence of $\left\{R_{n}\right\}_{n \in \mathbb{N}}$, if necessary, we assume without loss of generality that all $x_{n}$ are contained in the same face, say $F$, of $Q^{d}$ and the sequence $\left\{x_{n}\right\}_{n \in \mathbb{N}}$ converges to a point $\tilde{x} \in F$. 
First, let us consider $x \notin F$. Then

$$
0<\delta=: \operatorname{dist}_{\infty}(x, F) \leq \underline{\lim _{n \rightarrow \infty}}\left\|x-x_{n}\right\|_{\ell_{\infty}^{d}}=\|x-\tilde{x}\|_{\ell_{\infty}^{d}}
$$

In particular, $(x, \tilde{x}]$ is a nontrivial interval in $R$, i.e., $R \cap Q^{d} \neq \emptyset$ in this case.

Now let $x \in F$. Then all intervals $I_{n}$ are contained in $F$ as well. This reduces the problem to the case of the $(d-1)$-dimensional cube $F$ and the sequence of rays $\left\{R_{n}\right\}_{n \in \mathbb{N}}$ such that $R_{n} \cap F \backslash\{x\} \neq \emptyset$ for all $n$. Clearly, the limit ray $R$ belongs to the hyperplane containing $F$ and, hence, by the induction hypothesis $R \cap F \backslash\{x\} \neq \emptyset$.

This proves the lemma.

Finally, let us complete the proof of Theorem 2.1(b).

To this end, it remains to prove for $f \in \dot{V}_{p}^{k}$ that if $\left\{x_{n}\right\} \subset Q^{d} \backslash\{x\}$ is a sequence converging to $x$ such that $\lim _{n \rightarrow \infty} f\left(x_{n}\right)$ exists, then the limit coincides with the the radial limit of $f$ at $x$, see Lemma 3.7

Passing to a subsequence of $\left\{x_{n}\right\}_{n \in \mathbb{N}}$, if necessary, we assume without loss of generality that the rays $R_{n}$ with the origin at $x$ passing through $x_{n}, n \in \mathbb{N}$, converge to a ray $R$. By Proposition $3.8 R \cap\left(Q^{d} \backslash\{x\}\right) \neq \emptyset$. Then there is a hyperplane $H_{x}$ passing through $x$ orthogonal to a coordinate axis and an open half space $S \subset \mathbb{R}^{d}$ with the boundary $H_{x}$ such that $R \backslash\{x\} \subset S$. We show that for all sufficiently large $n$ points $x_{n}$ belong to $S$ as well.

In fact, otherwise the corresponding rays $R_{n}$ are located in the closed half space $\mathbb{R}^{d} \backslash S$ with the boundary $H_{x}$, hence, $R$ as the limit of the sequence $\left\{R_{n}\right\}_{n \in \mathbb{N}}$ is contained in $\mathbb{R}^{d} \backslash S$ as well, a contradiction.

Now, we proceed as in the proof of Lemma 3.6. Specifically, we draw hyperplanes through points $x_{n}$ parallel to $H_{x}$. They intersect $R$ at some points $y_{n}$. Then passing to a subsequence $\left\{x_{j}\right\}_{j \in J \subset \mathbb{N}}$ of $\left\{x_{n}\right\}_{n \in \mathbb{N}}$ we construct a sequence of nonoverlapping cubes $\left\{Q_{j}\right\}_{j \in J} \subset S \cap Q^{d} \backslash\{x\}$ converging to $\{x\}$ in the Hausdorff metric such that $\left[x_{j}, y_{j}\right] \subset Q_{j}$, $j \in J$, and

$$
\lim _{J \ni j \rightarrow \infty} \frac{\left\|x_{j}-y_{j}\right\|_{\ell_{\infty}^{d}}}{\operatorname{diam}\left(Q_{j}\right)}=0 .
$$

Using these and Lemma 3.1 and arguing as in the proof of Lemma 3.6 (i.e., applying the mean-value theorem and Markov inequality) we obtain that

$$
\lim _{J \ni j \rightarrow \infty}\left|f\left(x_{j}\right)-f\left(y_{j}\right)\right|=0 .
$$

This shows that $\lim _{n \rightarrow \infty} f\left(x_{n}\right)$ coincides with the radial limit of $f$ at $x$ and completes the proof of part (b) of the theorem.

(c) We should prove that if $s>k$, then the vector space $\dot{V}_{p}^{k}$ is the direct sum of subspaces $\ell_{p}$ and $\mathcal{P}_{k-1}^{d}$.

Due to parts (a) and (b) of the theorem, each $f \in \dot{V}_{p}^{k}$ is bounded and Lebesgue measurable. In particular, the map $L: \dot{V}_{p}^{k} \rightarrow L_{\infty}$ which sends a function to its equivalence class in $L_{\infty}$ is well defined. 
Lemma 3.9. It is true that range $(L) \subset \dot{V}_{p \infty}^{k}$ and the norm of $L: \dot{V}_{p}^{k} \rightarrow \dot{V}_{p \infty}^{k}$ is $\leq 1$.

Proof. Let us recall that $\dot{V}_{p \infty}^{k}$ is the subspace of $L_{\infty}$ functions on $Q^{d}$ equipped with the seminorm

$$
|f|_{V_{p \infty}^{k}}:=\sup _{\pi \in \Pi}\left(\sum_{Q \in \pi} E_{k \infty}(f ; Q)^{p}\right)^{\frac{1}{p}}
$$

where

$$
E_{k \infty}(f ; Q):=\inf _{m \in \mathcal{P}_{k-1}^{d}}\|f-m\|_{L_{\infty}(Q)} .
$$

Since for each Lebesgue measurable function $f \in \ell_{\infty}, E_{k \infty}(f ; Q) \leq E_{k}(f ; Q)$, the required statement follows directly from the definitions of seminorms of $\dot{V}_{p}^{k}$ and $\dot{V}_{p \infty}^{k}$.

Further, since $s>k$, Lemma 3.1 in [BB-18] implies that each $L(f)$ equals a polynomial, say $m_{f} \in \mathcal{P}_{k-1}^{d}$, a.e. on $Q^{d}$. Since by the definition of the map $L$ for every $m \in \mathcal{P}_{k-1}^{d}$ function $L(m)$ equals $m$ a.e. on $Q^{d}$, map $L: \dot{V}_{p}^{k} \rightarrow \dot{V}_{p \infty}^{k}$ is surjective and $\left.L\right|_{\mathcal{P}_{k-1}^{d}}: \mathcal{P}_{k-1}^{d} \rightarrow$ $\dot{V}_{p \infty}^{k}$ is an isomorphism; hence, $\operatorname{ker}(L) \cap \mathcal{P}_{k-1}^{d}=(0)$. This implies that $\dot{V}_{p}^{k}$ is isomorphic to the direct sum of $\operatorname{ker}(L)$ and $\mathcal{P}_{k-1}^{d}$. To complete the proof of part (c) it remains to show the following.

Lemma 3.10. $\operatorname{ker}(L)$ coincides with $\ell_{p}$.

Proof. First, let us show that $\ell_{p} \subset \dot{V}_{p}^{k}$.

In fact, we have for $f \in \ell_{p}$

$$
|f|_{V_{p}^{k}}:=\sup _{\pi \in \Pi}\left(\sum_{Q \in \pi} E_{k}(f ; Q)^{p}\right)^{\frac{1}{p}} \leq\left(\sum_{Q \in \pi}\left\{\sup _{Q}|f|\right\}^{p}\right)^{\frac{1}{p}} \leq\|f\|_{p}
$$

i.e., $f \in \dot{V}_{p}^{k}$ as required.

Clearly, $L(f)=0$ for each $f \in \ell_{p}$, i.e., $\ell_{p} \subset \operatorname{ker}(L)$.

Conversely, if $f \in \operatorname{ker}(L)$, then $f$ equals 0 a.e. on $Q^{d}$ and, moreover, this and Lemmas 3.1, 3.2 imply that supp $f$ is an at most countable subset of $Q^{d}$.

Let $S \subset \operatorname{supp} f$ be a finite subset. By definition the function

$$
f_{S}(y):=\left\{\begin{array}{cll}
0 & \text { if } & y \in S \\
f(y) & \text { if } & y \notin S
\end{array}\right.
$$

is continuous at each point of $S$. Due to Proposition 3.3 we then have, see (3.1),

$$
\left(f-f_{S}\right)_{k}(y) \leq f_{k}(y)+\left(f_{S}\right)_{k}(y)=f_{k}(y) \text { for all } y \in S .
$$

On the other hand, $f-f_{S}$ is supported by $S$ and $\left(f-f_{S}\right)(y)=f(y)$ for each $y \in S$. The direct computation of the limit in (3.1) gives for this case

$$
\left(f-f_{S}\right)_{k}(y)=\frac{1}{2}|f(y)|, \quad y \in S .
$$


Now (3.12), (3.13) and Lemma 3.2 imply that

$$
\frac{1}{2}\left(\sum_{y \in S}|f(y)|^{p}\right)^{\frac{1}{p}}=\left\|\left(f-f_{S}\right)_{k}\right\|_{p} \leq\left\|f_{k}\right\|_{p} \leq|f|_{V_{p}^{k}}
$$

This shows that $f \in \ell_{p}$ and $\|f\|_{p} \leq 2|f|_{V_{p}^{k}}$ and implies that $\operatorname{ker}(L) \subset \ell_{p}$.

The proof of Theorem 2.1 is complete.

\subsection{Proof of Corollary 2.2.}

Proof. (a) We begin with the case of $d \geq 2$. Then by definition

$$
\hat{f}(x):=\lim _{y \rightarrow x} f(y), \quad x \in Q^{d}
$$

and $P$ maps $f \in \dot{V}_{p}^{k}$ to $\hat{f}$.

We should prove that $P$ is a linear projection of norm 1 onto the closed subspace $C \cap \dot{V}_{p}^{k}$ denoted by $N \dot{V}_{p}^{k}$.

First we show that for $f \in \dot{V}_{p}^{k}$ the function $\hat{f} \in \dot{V}_{p}^{k}$ and $|\hat{f}|_{V_{p}^{k}} \leq|f|_{V_{p}^{k}}$. Since clearly $P \hat{f}=\hat{f}$ and $C^{\infty} \subset N \dot{V}_{p}^{k}$ (see the proof of Theorem [2.6] in Section 5), this will prove the corollary for this case.

To this end, it suffices to prove that for each closed cube $Q \subset Q^{d}$

$$
E_{k}(\hat{f} ; Q) \leq E_{k}(f ; Q) .
$$

Let $m \in \mathcal{P}_{k-1}^{d}$ be such that

$$
E_{k}(f ; Q)=\|f-m ; Q\|_{\infty} .
$$

Since by Theorem 2.1 function $\hat{f}$ is continuous on $Q^{d}$ and $f-\hat{f}$ has at most countable support, there are $x \in Q^{d}$ such that

$$
|\hat{f}(x)-m(x)|=\|\hat{f}-m ; Q\|_{\infty}
$$

and a sequence $\left\{x_{n}\right\}_{n \in \mathbb{N}} \not \subset \operatorname{supp}(f-\hat{f})$ in $Q$ converging to $x$. Hence, due to the continuity of $\hat{f}$,

$$
\lim _{n \rightarrow \infty}\left|\hat{f}\left(x_{n}\right)-m\left(x_{n}\right)\right|=|\hat{f}(x)-m(x)| .
$$

Using (3.15), (3.16) and (3.17), and the definition of $\hat{f}$ we obtain

$$
E_{k}(\hat{f} ; Q) \leq\|\hat{f}-m ; Q\|_{\infty}=\lim _{n \rightarrow \infty}\left|\hat{f}\left(x_{n}\right)-m\left(x_{n}\right)\right|=\lim _{n \rightarrow \infty}\left|f\left(x_{n}\right)-m\left(x_{n}\right)\right| \leq E_{k}(f ; Q)
$$

proving (3.14) and assertion (a) in this case.

Now let $d=1$, hence, $m$ in (3.15) belongs to $\mathcal{P}_{k-1}^{d}$ and $Q$ is a closed interval in $[0,1]$. Moreover, $\hat{f}(x):=f(x)$ if $f$ is a point of continuity for $f$, and $\hat{f}(x):=\frac{1}{2}\left(f\left(x^{-}\right)+f\left(x^{+}\right)\right)$for the remaining at most countable set of points $x$ in $(0,1)$ (with the corresponding correction for $x=0,1)$. As in the previous case, we show that (3.14) holds for $d=1$. 
Let $\left\{x_{n}\right\}_{n \in \mathbb{N}} \subset Q$ be such that

$$
\lim _{n \rightarrow \infty}\left|\hat{f}\left(x_{n}\right)-m\left(x_{n}\right)\right|=\|\hat{f}-m ; Q\|_{\infty}
$$

If $\left\{x_{n}\right\}_{n \in \mathbb{N}}$ contains an infinite subsequence of points of continuity of $\hat{f}$, then we can slightly change the latter to get a sequence outside of $\operatorname{supp}(f-\hat{f})$ (which is at most countable) satisfying (3.18). In this case the argument of the proof of the previous case gives the required inequality (3.14).

Otherwise, $\left\{x_{n}\right\}_{n \in \mathbb{N}}$ contains an infinite subsequence, say $\left\{x_{n_{k}}\right\}_{k \in \mathbb{N}}$, of points of discontinuity for $f$ in $(0,1)$. Then by the definition of $\hat{f}$ we derive from (3.18):

$$
\begin{aligned}
E_{k}(\hat{f} ; Q) & \leq\|\hat{f}-m ; Q\|_{\infty}=\lim _{k \rightarrow \infty}\left|\hat{f}\left(x_{n_{k}}\right)-m\left(x_{n_{k}}\right)\right| \\
& =\lim _{k \rightarrow \infty}\left|\frac{f\left(x_{n_{k}}^{-}\right)-m\left(x_{n_{k}}\right)}{2}+\frac{f\left(x_{n_{k}}^{+}\right)-m\left(x_{n_{k}}\right)}{2}\right| \leq\|f-m ; Q\|_{\infty}=E(f ; Q),
\end{aligned}
$$

as required.

Now, let $x_{n}=0$ for all $n \in \mathbb{N}$. Then we have by (3.18) and the definition of $\hat{f}$

$E_{k}(\hat{f} ; Q) \leq\|\hat{f}-m ; Q\|_{\infty}=|\hat{f}(0)-m(0)|:=\lim _{x \rightarrow 0^{+}}|f(x)-m(x)| \leq\|f-m ; Q\|_{\infty}=E(f ; Q)$.

The case of $x_{n}=1$ for all $n \in \mathbb{N}$ is considered similarly.

The proof of (3.14) is complete, i.e., $P: \dot{V}_{p}^{k} \rightarrow N \dot{V}_{p}^{k}\left(\subset \dot{V}_{p}^{k}\right)$ is a linear projection of norm 1 also for $d=1$.

(b) By the definition of the map $P$ its kernel contains functions in $\dot{V}_{p}^{k}$ with at most countable supports. Then as in the proof of part (c) of Theorem 2.1 we obtain that $\operatorname{ker}(P)=\ell_{p}$. Moreover, we have proved in Lemma 3.10 that for each $f \in \ell_{p}$

$$
|f|_{V_{p}^{k}} \leq\|f\|_{p} \leq 2|f|_{V_{p}^{k}}
$$

This shows that the identity map embeds $\ell_{p}$ into $\dot{V}_{p}^{k}$ as a Banach subspace.

The proof of the corollary is complete.

\section{Proofs of Theorems 2.3, 2.5] and Corollary 2.4}

In the proofs, we use the duality between $V_{p}^{k}$ and $U_{p}^{k}$ established in Theorem 2.25] and between $V_{p \infty}^{k}$ and $U_{p \infty}^{k}$ established in [BB-18, Th. 2.6].

\subsection{Auxiliary Results.}

4.1.1. In what follows, we fix an interpolating subset $S \subset Q^{d}$ for polynomials of $\mathcal{P}_{k-1}^{d}$. Hence, card $S=\operatorname{dim} \mathcal{P}_{k-1}^{d}+1$ and for each function $f \in \ell_{\infty}(S)$ there is a unique polynomial $m_{f} \in \mathcal{P}_{k-1}^{d}$ such that $\left.m_{f}\right|_{S}=f$. The norm of the linear operator $f \mapsto m_{f}$ is clearly bounded by a constant $c=c(S)$.

Let $\dot{V}_{p ; S}^{k} \subset \dot{V}_{p}^{k}$ denote the subspace of functions vanishing on $S$. Clearly, codim $\dot{V}_{p ; S}^{k}=$ card $S$ and for each $f \in \dot{V}_{p}^{k}$ we have the unique representation $f=m_{f}+\left(f-m_{f}\right)$ where 
$f-m_{f} \in \dot{V}_{p ; S}^{k}$. This implies that $\dot{V}_{p ; S}^{k}$ equipped with the seminorm $|\cdot|_{V_{p}^{k}}$ is a Banach space such that

$$
\dot{V}_{p ; S}^{k} \equiv V_{p}^{k}
$$

where the isometry is given by the restriction of the quotient map $q: \dot{V}_{p}^{k} \rightarrow V_{p}^{k}(:=$ $\dot{V}_{p}^{k} / \mathcal{P}_{k-1}^{d}$ ) to $\dot{V}_{p ; S}^{k}$. We equip $\dot{V}_{p ; S}^{k}$ with the weak* topology pulled back from that of $V_{p}^{k}$ by this isometry. The weak* topology on $V_{p}^{k}$ is defined by the duality relation

$$
V_{p}^{k} \equiv\left(U_{p}^{k}\right)^{*}
$$

that will be proved in Theorem 2.25.

Lemma 4.1. The closed unit ball $B\left(\dot{V}_{p ; S}^{k}\right)$ is compact in the topology of pointwise convergence on $Q^{d}$.

Proof. Since $B\left(\dot{V}_{p ; S}^{k}\right)$ is contained in $B\left(\ell_{\infty}\right)=[-1,1]^{Q^{d}}$ which is compact in the topology of pointwise convergence by the Tychonoff theorem, $B\left(\dot{V}_{p ; S}^{k}\right)$ is relatively compact in this topology. If now $\left\{f_{\alpha}\right\}_{\alpha \in \Lambda}$ is a net in $B\left(\dot{V}_{p ; S}^{k}\right)$ converging in the topology of pointwise convergence to a function $f \in \ell_{\infty}$, then we must show that $f \in B\left(\dot{V}_{p ; S}^{k}\right)$.

Since by the Banach-Alaoglu theorem $B\left(\dot{V}_{p ; S}^{k}\right)$ is weak* compact, see (4.2), we may assume, passing if necessary to a subnet of $\left\{f_{\alpha}\right\}_{\alpha \in \Lambda}$, that $\left\{f_{\alpha}\right\}_{\alpha \in \Lambda}$ converges in the weak* topology to some $\tilde{f} \in B\left(\dot{V}_{p ; S}^{k}\right)$.

Let us show that $f=\tilde{f}$.

In fact, let $\delta_{x}$ be the evaluation functional at $x \in Q^{d}$. By the definition of $S$, there exists an element $c_{x} \in \ell_{1}(S)$ such that

$$
m(x)=\sum_{s \in S} c_{x}(s) m(s) \quad \text { for all } \quad m \in \mathcal{P}_{k-1}^{d} .
$$

Hence, $\delta_{x}^{\prime}:=\delta_{x}-\sum_{s \in S} c_{x}(s) \delta(s)$ regarded as a function of $\ell_{1}$ supported by $S \cup\{x\}$ is orthogonal to $\mathcal{P}_{k-1}^{d}$; hence, $\delta_{x}^{\prime}$ belongs to $\left(U_{p}^{k}\right)^{0} \subset U_{p}^{k}$, see (2.33).

Now the weak* convergence of $\left\{f_{\alpha}\right\}_{\alpha \in \Lambda}$ in $B\left(\dot{V}_{p ; S}^{k}\right)$ and its pointwise convergence to $f$ imply for each $x \in Q^{d}$,

$$
\tilde{f}(x)=\left\langle f, \delta_{x}^{\prime}\right\rangle=\lim _{\alpha}\left\langle f_{\alpha}, \delta_{x}^{\prime}\right\rangle=\lim _{\alpha} f_{\alpha}(x)=f(x) .
$$

Hence, $\tilde{f}=f$, as required.

4.1.2. In the next proofs, we use the approximation theorem from [BB-18, Th. 2.12] asserting that for every $g \in \dot{V}_{p \infty}^{k}$ there is a sequence $\left\{g_{n}\right\}_{n \in \mathbb{N}} \subset C^{\infty}$ such that

$$
\lim _{n \rightarrow \infty}\left|g_{n}\right|_{V_{p \infty}^{k}}=|g|_{V_{p \infty}^{k}}
$$




$$
\lim _{n \rightarrow \infty} \int_{Q^{d}}\left(g-g_{n}\right) h d x=0 \quad \text { for each } \quad h \in L_{1} .
$$

The sequence $\left\{g_{n}\right\}_{n \in \mathbb{N}}$ is defined as follows.

Let $\varphi \in C^{\infty}\left(\mathbb{R}^{d}\right)$ be a function supported by the unit cube $[-1,1]^{d}$ such that

$$
0 \leq \varphi \leq 1 \quad \text { and } \quad \int_{\mathbb{R}^{d}} \varphi d x=1
$$

We denote by $g^{0}$ the extension of $g \in L_{\infty}$ by 0 outside $Q^{d}$ and then define $g_{n}^{0}, n \in \mathbb{N}$, by

$$
g_{n}^{0}(x):=\int_{\|y\|_{\ell_{\infty}^{d}} \leq 1} g^{0}\left(x-\frac{y}{n+1}\right) \varphi(y) d y, \quad x \in \mathbb{R}^{d} .
$$

Next, let $a_{n}: \mathbb{R}^{d} \rightarrow \mathbb{R}^{d}$ be the $\lambda_{n}$-dilation with the center $c$, where $\lambda_{n}:=\frac{n-1}{n+1}$ and $c$ is the center of $Q^{d}$.

Then $a_{n}\left(Q^{d}\right)$ is a subcube of $Q^{d}$ centered at $c_{Q^{d}}$ and

$$
\operatorname{dist}_{\ell_{\infty}^{d}}\left(\partial Q^{d}, a_{n}\left(Q^{d}\right)\right)=\frac{1}{n+1}
$$

We define $g_{n}:=T_{n}(g) \in C^{\infty}$ by setting

$$
g_{n}=g_{n}^{0} \circ a_{n} .
$$

4.2. Proof of Theorem 2.3. Let $f \in \dot{V}_{p}^{k}$. We should find a sequence $\left\{f_{n}\right\}_{n \in \mathbb{N}} \subset C^{\infty}$ satisfying the limit relations (2.2), (2.3).

Let $L: \dot{V}_{p}^{k} \rightarrow L_{\infty}$ be the map sending a function of $\dot{V}_{p}^{k}$ to its equivalence class in $L_{\infty}$. Since

$$
\|L(f)\|_{L_{\infty}(Q)} \leq\|f\|_{\ell_{\infty}(Q)} \quad \text { for all } \quad f \in \dot{V}_{p}^{k}, \quad Q \subset Q^{d},
$$

the range of $L$ is a subspace of $\dot{V}_{p \infty}^{k}$ and the linear map $L: \dot{V}_{p}^{k} \rightarrow \dot{V}_{p \infty}^{k}$ is bounded of norm $\leq 1$, cf. Lemma 3.9.

Let $f \in \dot{V}_{p}^{k}$ and $g:=L(f) \in \dot{V}_{p \infty}^{k}$. Then there is a sequence $\left\{g_{n}\right\}_{n \in \mathbb{N}} \subset C^{\infty}$ satisfying conditions (1), (2) of Section 4.1.2.

Further, $L$ maps $C^{\infty} \subset \dot{V}_{p}^{k}$ isometrically onto $C^{\infty} \subset \dot{V}_{p \infty}^{k}$ (because $E_{k \infty}(f ; Q)=$ $E_{k}(f ; Q)$ for all $f \in C^{\infty}$, cf. the argument of the proof of Lemma [3.9) . In particular, there exists a sequence $\left\{\tilde{f}_{n}\right\}_{n \in \mathbb{N}} \subset C^{\infty} \subset \dot{V}_{p}^{k}$ such that $L\left(\tilde{f}_{n}\right)=g_{n}$ for all $n$ and

$$
\lim _{n \rightarrow \infty}\left|\tilde{f}_{n}\right|_{V_{p}^{k}}=\lim _{n \rightarrow \infty}\left|g_{n}\right|_{V_{p \infty}^{k}}=|g|_{V_{p \infty}^{k}} \leq|f|_{V_{p}^{k}}
$$

Lemma 4.2. For each point of continuity $x \in Q^{d}$ of $f$

$$
\lim _{n \rightarrow \infty} \tilde{f}_{n}(x)=f(x) \text {. }
$$


Proof. We have by the definitions of $L, a_{n}, g_{n}, \tilde{f}_{n}$, see (4.3) - (4.5),

$$
\begin{aligned}
& \left|\tilde{f}_{n}(x)-f(x)\right|=\left|\int_{\|y\|_{\ell_{\infty}^{d}} \leq 1}\left(f^{0}\left(a_{n}(x)-\frac{y}{n+1}\right)-f(x)\right) \varphi(y) d y\right| \\
& =\left|\int_{\|y\|_{\ell_{\infty}^{d}} \leq 1}\left(f\left(a_{n}(x)-\frac{y}{n+1}\right)-f(x)\right) \varphi(y) d y\right| \leq \sup _{\|y\|_{\ell_{\infty}^{d}}}\left|f\left(a_{n}(x)-\frac{y}{n+1}\right)-f(x)\right| .
\end{aligned}
$$

Since $a_{n}(x) \rightarrow x$ as $n \rightarrow \infty$, the last term in (4.8) tends to 0 as $n \rightarrow \infty$ by continuity of $f$ at $x$.

The lemma is proved.

Further, by the definition of the sequence $\left\{g_{n}\right\}_{n \in \mathbb{N}}$, see (4.3), (4.5),

$$
\left\|\tilde{f}_{n}\right\|_{\infty} \leq\|g\|_{L_{\infty}}\left(\leq\|f\|_{\infty}\right) \text { for all } n \in \mathbb{N}
$$

Since by Theorem 2.1 the set $S_{f}$ of discontinuities of $f$ is at most countable, the Cantor diagonal procedure gives a subsequence $\left\{\tilde{f}_{n_{k}}\right\}_{k \in \mathbb{N}}$ of $\left\{\tilde{f}_{n}\right\}_{n \in \mathbb{N}}$ such that the limit

$$
\lim _{k \rightarrow \infty} \tilde{f}_{n_{k}}(x) \leq\|g\|_{L_{\infty}}
$$

exists for all $x \in S_{f}$.

We define $\tilde{f} \in \ell_{\infty}$ as the pointwise limit of the sequence $\left\{\tilde{f}_{n_{k}}\right\}_{k \in \mathbb{N}}$ (in particular, $\tilde{f}$ coincides with $f$ at the points of continuity of $f$ ).

Lemma 4.3. $\tilde{f} \in \dot{V}_{p}^{k}$ and $|\tilde{f}|_{V_{p}^{k}} \leq|f|_{V_{p}^{k}}$.

Proof. We set for brevity

$$
h_{k}:=\tilde{f}_{n_{k}}, \quad k \in \mathbb{N},
$$

and use the interpolating set $S \subset Q^{d}$ for $\mathcal{P}_{k-1}^{d}$ of Section 4.1.1 to decompose $h_{k}$ as follows

$$
h_{k}=m_{h_{k}}+\left(h_{k}-m_{h_{k}}\right),
$$

where $m_{h_{k}} \subset \mathcal{P}_{k-1}^{d}$ interpolates $h_{k}$ on $S$.

The second term belongs to the space $\dot{V}_{p ; S}^{k}$ consisting of all functions from $\dot{V}_{p}^{k}$ vanishing on $S$ so that $\left|h_{k}-m_{h_{k}}\right|_{V_{p}^{k}}=\left|h_{k}\right|_{V_{p}^{k}}$. Further, due to (4.9)

$$
\left\|m_{h_{k}} ; S\right\|_{\infty} \leq\|g\|_{L_{\infty}} .
$$

Hence, the sequence $\left\{m_{h_{k}}\right\}_{k \in \mathbb{N}}$ is uniformly bounded on $Q^{d}$ and, in particular, it contains a uniformly converging subsequence. Passing to such a subsequence, if necessary, without loss of generality we will assume that $\left\{m_{h_{k}}\right\}_{k \in \mathbb{N}}$ itself converges uniformly to a polynomial $m \in \mathcal{P}_{k-1}^{d}$. Therefore the sequence $\left\{h_{k}-m_{h_{k}}\right\}_{k \in \mathbb{N}}$ is uniformly bounded and pointwise converges to $\tilde{f}-m \in \ell_{\infty}$. According to Lemma 4.1, the closed ball $B\left(\dot{V}_{p ; S}^{k}\right)$ is compact in the topology of pointwise convergence on $Q^{d}$. Hence, $\tilde{f}-m \in V_{p ; S}^{k}$ and by (4.6)

$$
|\tilde{f}|_{V_{p}^{k}}=|\tilde{f}-m|_{V_{p}^{k}} \leq \varlimsup_{k \rightarrow \infty}\left|h_{k}-m_{h_{k}}\right|_{V_{p}^{k}}=\varlimsup_{k \rightarrow \infty}\left|h_{k}\right|_{V_{p}^{k}} \leq|f|_{V_{p}^{k}}
$$


The proof of the lemma is complete.

Now set

$$
l:=f-\tilde{f} .
$$

Since $\tilde{f}$ coincides with $f$ on the continuity set of $f$, see Lemma 4.2, function $l \in \ell_{p}$ and by Lemma 4.3 ,

$$
|l|_{V_{p}^{k}} \leq 2|f|_{V_{p}^{k}}
$$

Lemma 4.4. Let $l \in \ell_{p}$. There is a sequence $\left\{l_{n}\right\}_{n \in \mathbb{N}} \subset C^{\infty}$ pointwise converging to $l$ such that

$$
\sup _{n \in \mathbb{N}}\left\|l_{n}\right\|_{\infty} \leq\|l\|_{\infty} \quad \text { and } \quad \varlimsup_{n \rightarrow \infty}\left|l_{n}\right|_{V_{p}^{k}} \leq\|l\|_{p} .
$$

Proof. Since the set of functions with finite supports is dense in $\ell_{p}$, without loss of generality we may assume that $l$ is supported by a finite set, say $\left\{x_{1}, \ldots, x_{m}\right\} \subset Q^{d}$. In this case, Euclidean balls of radius $r$ centered at points $x_{i}$ are mutually disjoint for some $r>0$.

Let $s_{j n}$ be a $C^{\infty}$ function supported by the Euclidean ball $B_{\frac{r}{n}}\left(x_{j}\right)$ such that $s_{j i}\left(x_{j}\right)=1$ and $0 \leq s_{j i} \leq 1, n \in \mathbb{N}, 1 \leq j \leq m$.

We set

$$
l_{n}:=\sum_{l=1}^{m} l\left(x_{j}\right) s_{j n} .
$$

By the definition, for a nontrivial closed cube $Q \subset Q^{d}$

$$
E_{k}\left(l_{n} ; Q\right) \leq\left\|l_{n} ; Q\right\|_{\infty} \leq\|l ; Q\|_{\infty} \leq\left(\sum_{x_{i} \in Q}\left|l\left(x_{i}\right)\right|^{p}\right)^{\frac{1}{p}} .
$$

This implies that for all $n$

$$
\left\|l_{n}\right\|_{\infty} \leq\|l\|_{\infty} \quad \text { and } \quad\left|l_{n}\right|_{V_{p}^{k}} \leq\|l\|_{p}
$$

and proves the required inequalities.

It remains to prove that

$$
\lim _{n \rightarrow \infty}\left(l(y)-l_{n}(y)\right)=0 \text { for all } y \in Q^{d} .
$$

Indeed, if $y$ coincides with some $x_{j}, 1 \leq j \leq m$, then

$$
l_{n}(y)=l(y) \text { for all } n
$$

and the result follows.

Otherwise, $l(y)=0$ and there is some $n_{0} \in \mathbb{N}$ such that $y \notin \operatorname{supp} l_{n}$ for all $n \geq n_{0}$. Hence, $l_{n}(y)=0$ for such $n$ and

$$
\lim _{n \rightarrow \infty}\left(l(y)-l_{n}(y)\right)=0
$$

as well.

The proof is complete. 
Let $\left\{l_{k}\right\}_{k \in \mathbb{N}} \subset C^{\infty}$ and $\left\{h_{k}\right\}_{k \in \mathbb{N}} \subset C^{\infty}$ be as above, see (4.13), (4.10). Then we set

$$
f_{n}:=h_{n}+l_{n}, \quad n \in \mathbb{N} .
$$

and show that $\left\{f_{k}\right\}_{k \in \mathbb{N}}$ is the required sequence.

In fact, by (4.9), (4.11) and (4.13)

$$
\varlimsup_{n \rightarrow \infty}\left\|f_{n}\right\|_{\infty} \leq \varlimsup_{n \rightarrow \infty}\left\|h_{n}\right\|_{\infty}+\varlimsup_{n \rightarrow \infty}\left\|l_{n}\right\|_{\infty} \leq\|f\|_{\infty}+\|f-\tilde{f}\|_{\infty} \leq 3\|f\|_{\infty} .
$$

This give the first inequality (2.2).

Further, by (4.6) and (4.13)

$$
\varlimsup_{n \rightarrow \infty}\left\|h_{n}\right\|_{V_{p}^{k}}+\varlimsup_{n \rightarrow \infty}\left\|l_{n}\right\|_{V_{p}^{k}} \leq\|f\|_{V_{p}^{k}}+\|l\|_{p} .
$$

Moreover, applying the inequality proved in Lemma 3.10 and (4.11) we get

$$
\|l\|_{p} \leq 2|l|_{V_{p}^{k}} \leq 4|f|_{V_{p}^{k}}
$$

Combining these inequalities we finally have

$$
\varlimsup_{n \rightarrow \infty}\left\|f_{n}\right\|_{V_{p}^{k}} \leq 5\|f\|_{V_{p}^{k}}
$$

This proves the second inequality (2.2).

Further, by its definition the sequence $\left\{f_{k}\right\}_{k \in \mathbb{N}} \subset C^{\infty}$ converges pointwise to $f$, hence, belongs to the first Baire class, and is uniformly bounded. By the Rosenthal Main Theorem Ro-77] every such sequence satisfies

$$
\lim _{k \rightarrow \infty} \int_{Q^{d}}\left(f-f_{k}\right) d \mu=0
$$

for all measures $\mu \in M$.

This proves (2.3) and completes the proof of the theorem.

4.3. Proof of Corollary 2.4. We retain notation of Section 4.1.1.

Let $\left\{f_{n}\right\}_{n \in \mathbb{N}} \subset \dot{V}_{p}^{k}$ be bounded. We choose a subsequence $\left\{f_{n_{i}}\right\}_{i \in \mathbb{N}}$ such that

$$
\lim _{i \rightarrow \infty}\left|f_{n_{i}}\right|_{V_{p}^{k}}=\varliminf_{n \rightarrow \infty}\left|f_{n}\right|_{V_{p}^{k}} .
$$

Next, we write

$$
f_{n_{i}}=m_{f_{n_{i}}}+\left(f_{n_{i}}-m_{f_{n_{i}}}\right),
$$

where $m_{f_{n_{i}}} \in \mathcal{P}_{k-1}^{d}, f_{n_{i}}-m_{f_{n_{i}}} \in \dot{V}_{p ; S}^{k}, i \in \mathbb{N}$.

By the definition,

$$
\sup _{i \in \mathbb{N}}\left|f_{n_{i}}-m_{f_{n_{i}}}\right|_{V_{p}^{k}}=\sup _{i \in \mathbb{N}}\left|f_{n_{i}}\right|_{V_{p}^{k}} \leq \sup _{n \in \mathbb{N}}\left|f_{n}\right|_{V_{p}^{k}}=: r<\infty .
$$

Hence, the sequence $\left\{f_{n_{i}}-m_{f_{n_{i}}}\right\}_{i \in \mathbb{N}}$ belongs to the closed ball of $\dot{V}_{p ; S}^{k}$ of radius $r$ centered at 0 and so by Lemma 4.1 is relatively compact in the ball equipped with the topology of pointwise convergence on $Q^{d}$. In addition, by Theorem 2.3 this sequence is in the first Baire class. Therefore the Rosenthal Main Theorem [Ro-77] imply that it contains a pointwise 
convergent subsequence $\left\{f_{n_{j}}-m_{f_{n_{j}}}\right\}_{j \in J}, J \subset \mathbb{N}$. Due to (4.17) its limit, say $f \in \dot{V}_{S ; p}^{k}$, belongs to the ball of radius $\underline{\lim }_{n \in \mathbb{N}}\left|f_{n}\right|_{V_{p}^{k}}$.

This completes the proof of the corollary.

4.4. Proof of Theorem 2.5. (a) Let as above $L: \dot{V}_{p}^{k} \rightarrow L_{\infty}$ be the map sending a function in $\dot{V}_{p}^{k}$ to its equivalence class in $L_{\infty}$. It was shown in Section 4.2 that the range of $L$ is a subset of $\dot{V}_{p \infty}^{k}$ and the linear map $L: \dot{V}_{p}^{k} \rightarrow \dot{V}_{p \infty}^{k}$ has norm $\leq 1$. We have to check that the range of $L$ is $\dot{V}_{p \infty}^{k}$ and that $\|L\|=1$. that

Let $f \in \dot{V}_{p \infty}^{k}$. Due to [BB-18, Th. 2.12] there is a sequence $\left\{g_{n}\right\}_{n \in \mathbb{N}} \subset C^{\infty} \subset \dot{V}_{p}^{k}$ such

$$
\sup _{n \in \mathbb{N}}\left\|g_{n}\right\|_{\infty} \leq\|f\|_{L_{\infty}} \quad \text { and } \quad \lim _{n \rightarrow \infty}\left|g_{n}\right|_{V_{p}^{k}}=|f|_{V_{p \infty}^{k}},
$$

and, moreover, $\left\{L\left(g_{n}\right)\right\}_{n \in \mathbb{N}} \subset C^{\infty} \subset V_{p \infty}^{k}$ weak* $^{*}$ converges to $f$ in the weak* topology of $L_{\infty}$ defined by the duality $L_{1}^{*}=L_{\infty}$.

Using the interpolating set $S \subset Q^{d}$ for $\mathcal{P}_{k-1}^{d}$ of Section 4.1 .1 we decompose $g_{n}$ in a sum

$$
g_{n}=m_{g_{n}}+\left(g_{n}-m_{g_{n}}\right),
$$

where $m_{g_{n}} \subset \mathcal{P}_{k-1}^{d}$ interpolates $g_{n}$ on $S$ and the second term belongs to the space $\dot{V}_{p ; S}^{k}$ of functions from $\dot{V}_{p}^{k}$ vanishing on $S$ so that $\left|g_{n}-m_{g_{n}}\right|_{V_{p}^{k}}=\left|g_{n}\right|_{V_{p}^{k}}$.

According to (4.18) the sequence $\left\{m_{g_{n}}\right\}_{n \in \mathbb{N}}$ is uniformly bounded on the interpolating set $S$, hence, on $Q^{d}$. Passing to a subsequence of $\left\{g_{n}\right\}_{n \in \mathbb{N}}$, if necessary, without loss of generality we may assume that $\left\{m_{g_{n}}\right\}_{n \in \mathbb{N}}$ converges uniformly on $Q^{d}$ to some $m \in \mathcal{P}_{k-1}^{d}$. Moreover, (4.18) implies that

$$
\lim _{n \rightarrow \infty}\left|g_{n}-m_{g_{n}}\right|_{V_{p}^{k}}=|f|_{V_{p \infty}^{k}}
$$

i.e., the sequence $\left\{g_{n}-m_{g_{n}}\right\}_{n \in \mathbb{N}}$ is bounded in $\dot{V}_{p ; S}^{k}$. Using the weak ${ }^{*}$ topology on $\dot{V}_{p ; S}^{k}$ induced by the duality between $\dot{V}_{p ; S}^{k}\left(\equiv V_{p}^{k}\right)$ and $U_{p}^{k}$, we find a subnet $\left\{n_{\alpha}\right\}_{\alpha \in \Lambda}$ of $\mathbb{N}$ such that the subnet $\left\{g_{n_{\alpha}}-m_{h_{n_{\alpha}}}\right\}_{\alpha \in \Lambda}$ weak* converges to some $h \in \dot{V}_{p ; S}^{k}$. Since, in addition, the subnet $\left\{m_{g_{n_{\alpha}}}\right\}_{\alpha \in \Lambda}$ converges uniformly on $Q^{d}$ to $m$, the subnet $\left\{g_{n_{\alpha}}\right\}_{\alpha \in \Lambda}$ of $\left\{g_{n}\right\}_{n \in \mathbb{N}}$ pointwise converges to $g:=h+m$, cf. the argument of Lemma 4.1. Moreover, this subnet is uniformly bounded in $\ell_{\infty}$ by (4.18).

Hence, by the Rosenthal Main Theorem [Ro-77.

$$
\lim _{\alpha} \int_{Q^{d}} g_{n_{\alpha}} d \mu=\int_{Q^{d}} g d \mu
$$

for all measures $\mu \in M$.

Taking here $d \mu=h d x, h \in L_{1}$, we obtain that

$$
\lim _{\alpha} \int_{Q^{d}} g_{n_{\alpha}} h d x=\int_{Q^{d}} g h d x \quad \text { for all } h \in L_{1} .
$$


The latter implies that the net $\left\{L\left(g_{n_{\alpha}}\right)\right\}_{\alpha \in \Lambda}$ weak* converges to $L(g)$. Hence, by (4.18), $L(g)=f$.

This proves surjectivity of the map $L: \dot{V}_{p}^{k} \rightarrow \dot{V}_{p \infty}^{k}$.

Finally, by (4.18) and the inequality $\|L\| \leq 1$ we get

$$
|g|_{V_{p}^{k}}=|L(g)|_{V_{p \infty}^{k}} \leq|g|_{V_{p}^{k}}
$$

i.e., $|L(g)|_{V_{p \infty}^{k}}=|g|_{V_{p}^{k}}$. This implies that $\|L\|=1$.

The proof of part (a) of the theorem is complete.

(b) Now we should prove that $\operatorname{ker}(L)=\operatorname{ker}(P)=\ell_{p}$.

By the definition of the map $L$ its kernel consists of all functions in $\dot{V}_{p}^{k}$ which are zeros outside sets of Lebesgue measure zero. Then by Lemma $3.10 \operatorname{ker}(L)=\ell_{p}$. The same is established in Corollary 2.2 (b) for $\operatorname{ker}(P)$.

This completes the proof of this part of the theorem.

(c) It remains to show that $L$ maps $N \dot{V}_{p}^{k}$ isometrically onto $\dot{V}_{p \infty}^{k}$.

As in part (a) for each $f \in \dot{V}_{p \infty}^{k}$ we take $g \in \dot{V}_{p}^{k}$ such that $L(g)=f$ and $|g|_{V_{p}^{k}}=|f|_{V_{p \infty}^{k}}$. Then we set

$$
h:=P(g)\left(\in N \dot{V}_{p}^{k}\right) .
$$

Since $h-g \in \ell_{p}$, see Corollary 2.2, $\|L\|=1$ and $\|P\|=1$,

$$
L(h)=L(g)=f \quad \text { and } \quad|f|_{V_{p \infty}^{k}} \leq|h|_{V_{p}^{k}} \leq|g|_{V_{p}^{k}}=|f|_{V_{p \infty}^{k}} .
$$

Moreover, $\operatorname{ker}(L) \cap N \dot{V}_{p}^{k}=\ell_{p} \cap N \dot{V}_{p}^{k}=(0)$ by Corollary 2.2. These imply existence for each $f \in \dot{V}_{p \infty}^{k}$ a unique $h \in N \dot{V}_{p}^{k}$ such that $L(h)=f$ and $|h|_{V_{p}^{k}}=|f|_{V_{p \infty}^{k}}$.

Thus, $L: N \dot{V}_{p}^{k} \rightarrow V_{p \infty}^{k}$ is an isometric isomorphism of semi-Banach spaces.

The proof of Theorem 2.5] is complete.

\section{Proof of Theorem 2.6}

Let $\left(\dot{V}_{p}^{k}\right)^{0} \subset \dot{V}_{p}^{k}$ denote the subset of functions $f$ satisfying the condition

$$
\lim _{\varepsilon \rightarrow 0} \sup _{d(\pi) \leq \varepsilon}\left(\sum_{Q \in \pi} E_{k}(f ; Q)^{p}\right)^{\frac{1}{p}}=0 ;
$$

where $d(\pi):=\sup _{Q \in \pi}|Q|$.

We have to prove that if $k>s:=\frac{d}{p}$, then $\left(\dot{V}_{p}^{k}\right)^{0}=\dot{\mathrm{V}}_{p}^{k}$.

First, let us show that $\left(\dot{V}_{p}^{k}\right)^{0}$ is a closed subspace of the space $\dot{V}_{p}^{k}$.

To this end we define a seminorm $T: \dot{V}_{p}^{k} \rightarrow \mathbb{R}_{+}$given for $f \in \dot{V}_{p}^{k}$ by

$$
T(f):=\lim _{\varepsilon \rightarrow 0} \sup _{d(\pi) \leq \varepsilon}\left(\sum_{Q \in \pi} E_{k}(f ; Q)^{p}\right)^{\frac{1}{p}} .
$$


Since $T(f) \leq|f|_{V_{p}^{k}}$ for all $f \in \dot{V}_{p}^{k}$, seminorm $T$ is continuous on $\dot{V}_{p}^{k}$. This implies that the preimage $T^{-1}(\{0\})=\left(\dot{V}_{p}^{k}\right)^{0}$ is a closed subspace of $\dot{V}_{p}^{k}$.

Next, we show that $\dot{\mathrm{V}}_{p}^{k}$ is a closed subspace of $\left(\dot{V}_{p}^{k}\right)^{0}$.

Since $\dot{\mathrm{V}}_{p}^{k}=\operatorname{clos}\left(C^{\infty} \cap \dot{V}_{p}^{k}, \dot{V}_{p}^{k}\right)$ and $\left(\dot{V}_{p}^{k}\right)^{0}$ is closed in $\dot{V}_{p}^{k}$, it suffices to prove that $C^{\infty} \subset\left(\dot{V}_{p}^{k}\right)^{0}$.

To this end we estimate $E_{k}(f ; Q), Q \in \pi$, with $f \in C^{\infty}$ by the Taylor formula as follows

$$
E_{k}(f ; Q) \leq c(k, d)|Q|^{\frac{k}{d}} \max _{|\alpha|=k} \max _{Q}\left|D^{\alpha} f\right| \leq c(k, d, f)|Q|^{\frac{k}{d}} .
$$

This implies that

$$
\gamma(\pi ; f) \leq c\left(\sum_{Q \in \pi}|Q|^{\frac{k-s}{d} p+1}\right)^{\frac{1}{p}} \leq c \max _{Q \in \pi}|Q|^{\frac{k-s}{d}}\left(\sum_{Q \in \pi}|Q|\right)^{\frac{1}{p}}
$$

hereafter we set

$$
\gamma(\pi ; f):=\left(\sum_{Q \in \pi} E_{k}(f ; Q)^{p}\right)^{\frac{1}{p}}
$$

Since $s<k$ and the sum here $\leq 1$, this implies that

$$
\sup _{d(\pi) \leq \varepsilon} \gamma(\pi ; f) \leq c \varepsilon^{\frac{k-s}{d}} \rightarrow 0 \quad \text { as } \quad \varepsilon \rightarrow 0,
$$

i.e., $f \in\left(\dot{V}_{p}^{k}\right)^{0}$ as required.

Finally, let us prove that $\left(\dot{V}_{p}^{k}\right)^{0}=\dot{\mathrm{v}}_{p}^{k}$.

To this end we need the embedding

$$
\left(\dot{V}_{p}^{k}\right)^{0} \subset C
$$

proved, in fact, in [BB-18, Lm. 3.6].

Let us recall that as in this lemma the inequality

$$
\omega_{k}(f ; t) \leq \sup _{|Q| \leq(k t)^{d}} \operatorname{osc}_{k}(f ; Q) \leq 2^{d} \sup _{|Q| \leq(k t)^{d}} E_{k}(f ; Q) \leq 2^{d} \sup _{d(\pi) \leq(k t)^{d}} \gamma(\pi ; f)
$$

implies that for $f \in\left(\dot{V}_{p}^{k}\right)^{0}$

$$
\lim _{t \rightarrow 0} \omega_{k}(f ; t)=0
$$

while the Marchaud inequality, see, e.g., [BBI-11, Ch.2, App.E2], implies from here that $\omega_{1}(f ; t) \rightarrow 0$ as $t \rightarrow 0$, i.e., $f \in C$.

From (5.5) we obtain that $\left(\dot{V}_{p}^{k}\right)^{0} \subset N \dot{V}_{p}^{k}$, hence, due to Theorem 2.5](c) the operator $L: \dot{V}_{p}^{k} \rightarrow \dot{V}_{p \infty}^{k}$ (sending a function of $\dot{V}_{p}^{k}$ to its class of equivalence in $L_{\infty}$ ) embeds $\left(\dot{V}_{p}^{k}\right)^{0}$ isometrically into $\dot{V}_{p \infty}^{k}$. 
Further, since $L$ isometrically maps $C^{\infty}$ as a subset of $\dot{V}_{p}^{k}$ onto $C^{\infty}$ as that of $\dot{V}_{p \infty}^{k}$, it isometrically maps $\dot{\mathrm{V}}_{p}^{k}$ onto $\dot{\mathrm{V}}_{p \infty}^{k}\left(:=\operatorname{clos}\left(C^{\infty}, \dot{V}_{p \infty}^{k}\right)\right)$. In turn, by [BB-18, Thm. 2.13],

$$
\dot{\mathrm{V}}_{p \infty}^{k}=\left(\dot{V}_{p \infty}^{k}\right)^{0}
$$

where

$$
\begin{aligned}
& \left(\dot{V}_{p \infty}^{k}\right)^{0}:=\left\{f \in \dot{V}_{p \infty}^{k}: \lim _{\varepsilon \rightarrow 0} \sup _{|\pi| \leq \varepsilon}\left(\sum_{Q \in \pi} E_{k \infty}(f ; Q)^{p}\right)^{\frac{1}{p}}=0\right\} ; \\
& E_{k \infty}(f ; Q):=\inf _{m \in \mathcal{P}_{k-1}^{d}}\|f-m\|_{L_{\infty}(Q) .}
\end{aligned}
$$

Comparing the definitions of $\left(\dot{V}_{p}^{k}\right)^{0}(\subset C)$ and $\left(\dot{V}_{p \infty}^{k}\right)^{0}$ we see that $L\left(\left(\dot{V}_{p}^{k}\right)^{0}\right) \subset\left(\dot{V}_{p \infty}^{k}\right)^{0}$.

Since $\dot{\mathrm{V}}_{p}^{k} \subset\left(\dot{V}_{p}^{k}\right)^{0}$, the above implications yield

$$
L\left(\dot{\mathrm{V}}_{p}^{k}\right) \subset L\left(\left(\dot{V}_{p}^{k}\right)^{0}\right) \subset\left(\dot{V}_{p \infty}^{k}\right)^{0}=\dot{\mathrm{V}}_{p \infty}^{k}=L\left(\dot{\mathrm{V}}_{p}^{k}\right) .
$$

Hence, $L\left(\left(\dot{V}_{p}^{k}\right)^{0}\right)=L\left(\dot{\mathrm{V}}_{p}^{k}\right)$. This and the injectivity of $\left.L\right|_{\left(\dot{V}_{p}^{k}\right)^{0}}$ imply that

$$
\left(\dot{V}_{p}^{k}\right)^{0}=\dot{\mathrm{V}}_{p}^{k}
$$

The proof of the theorem is complete.

\section{Proof of Theorem 2.8}

The proof of the theorem is based on the following result of independent interest.

Theorem 6.1. Let $Q \Subset \mathbb{R}^{d}$ be a nontrivial closed cube and $f \in \ell_{\infty}(Q)$. Then the following two-sided inequality with equivalence constants depending only on $k, d$ holds:

$$
E_{k}(f ; Q) \approx \operatorname{osc}_{k}(f ; Q) \text {. }
$$

Proof. We begin with the proof of the inequality

$$
E_{k}(f ; Q) \leq c(k, d) \operatorname{osc}_{k}(f ; Q), \quad Q \subset Q^{d},
$$

where throughout the proof $c(k, d)$ denotes a positive constant depending on $k, d$ and changing from line to line or within a line.

Without loss of generality it suffices to prove (6.2) for $Q=Q^{d}$; in this case, we write in (6.2) $E_{k}(f)$ and $\operatorname{osc}_{k} f$ omitting $Q^{d}$, see Stipulation 1.10.

For $d=1$ the inequality was proved in Wh-59. In more details, let $f \in \ell_{\infty}[0,1]$ and $L_{k} f$ be a polynomial of degree $k-1$ interpolating $f$ at points $\frac{i}{k-1}, i=0, \ldots, k$, if $k>1$ and equal $\frac{1}{2}(f(0)+f(1))$ if $k=1$. Clearly, $L_{k}$ is a projection of $\ell_{\infty}[0,1]$ onto $\mathcal{P}_{k-1}^{1}$.

Now according to Wh-59]

$$
\left\|f-L_{k} f\right\|_{\infty} \leq \operatorname{cosc}_{k} f
$$

where $c=c(k)$.

Further, let $\mathscr{Q}_{k}^{i}$ be a linear subspace of $\ell_{\infty}$ consisting of polynomials in $x_{i}$ of degree $k-1$ with coefficients depending on the remaining variables $x_{j} \neq x_{i}$. This is extended to 
$k=0$ by setting $\mathscr{Q}^{d}=\{0\}$. Moreover, we define a projection of $\ell_{\infty}$ onto $\mathscr{Q}_{k}^{i}$ denoted by $L_{k}^{i}$ given for $f \in \ell_{\infty}$ by applying the interpolation operator $L_{k}$ to the function $x_{i} \mapsto f(x)$, $0 \leq x_{i} \leq 1$, with fixed variables $x_{j} \neq x_{i}$.

As a direct consequence of this definition and inequality (6.3) we have

$$
\left\|f-L_{k}^{i} f\right\|_{\infty} \leq \operatorname{cosc}_{k}^{i} f
$$

where $c=c(k)$ and

$$
\operatorname{osc}_{k}^{i} f:=\sup _{x, h}\left\{\left|\Delta_{h}^{k} f(x)\right|\right\}
$$

where supremum is taken for the $x, h \in \mathbb{R}^{d}$ satisfying the condition

$\left(^{*}\right) h$ is parallel to the $x_{i}$-axis and $x, x+k h \in Q^{d}$.

Further, again directly from the definition we have the following

Lemma 6.2. If $i \neq i^{\prime}$, then projections $L_{k}^{i}, L_{k^{\prime}}^{i^{\prime}}$ commute.

Now let $\alpha \in \mathbb{Z}_{+}^{d}$ and

$$
L_{\alpha}:=\prod_{i=1}^{d} L_{\alpha_{i}}^{i}
$$

Since the projections here pairwise commute, $L_{\alpha} f$ is a polynomial in $x_{i}$ of degree $\alpha_{i}-1$ for each $1 \leq i \leq d$.

Hence, $L_{\alpha} f$ is a polynomial of vector degree $\alpha-e$, where $e:=\{1, \ldots, 1\}$. The space of these polynomials is denoted by $\mathcal{P}_{\alpha}$.

Lemma 6.3. It is true that

$$
\left\|f-L_{\alpha} f\right\|_{\infty} \leq c \sum_{i=1}^{d} \operatorname{osc}_{\alpha_{i}}^{i} f
$$

where $c=c(\alpha, d)$.

Proof. Using the identity

$$
1-L_{\alpha}=\left(1-L_{\alpha_{1}}^{1}\right)+\sum_{i=2}^{d}\left(\prod_{j=1}^{i-1} L_{\alpha_{j}}^{j}\right)\left(1-L_{\alpha_{i}}^{i}\right),
$$

the estimate $\left\|L_{k}^{i}\right\| \leq\left\|L_{k}\right\|, 1 \leq i \leq d$, and Lemma 6.2 we conclude that

$$
\left\|f-L_{\alpha} f\right\|_{\infty} \leq\left(\prod_{i=1}^{d-1}\left\|L_{\alpha_{i}}\right\|\right) \sum_{i=1}^{d}\left\|f-L_{\alpha_{i}}^{i} f\right\|_{\infty} \leq c(\alpha, d) \sum_{i=1}^{d} \operatorname{osc}_{\alpha_{i}}^{i} f .
$$

Corollary 6.4. A function $f \in \ell_{\infty}$ belongs to the space $\mathcal{P}_{\alpha}$ if and only if

$$
\operatorname{osc}_{\alpha_{i}}^{i} f=0 \text { for all } 1 \leq i \leq d \text {. }
$$


Proof. If (6.8) holds, then by (6.7) $f=L_{\alpha} f \in \mathcal{P}_{\alpha}$.

Conversely, if $f \in \mathcal{P}_{\alpha}$, then $\Delta_{h}^{\alpha_{i}} f=0$ for every $h$ parallel to the $x_{i}$ axis, as $f$ is a polynomial in $x_{i}$ of degree $\alpha_{i}-1,1 \leq i \leq d$.

This and definition (6.5) imply (6.8).

At the next step, we define a subspace of $\ell_{\infty}$ by setting

$$
\mathscr{Q}_{\alpha}:=\sum_{i=1}^{d} \mathscr{Q}_{\alpha_{i}}^{i}
$$

and an operator acting in $\ell_{\infty}$ by

$$
\mathcal{L}_{\alpha}:=1-\prod_{i=1}^{d}\left(1-L_{\alpha_{i}}^{i}\right) .
$$

Since $1-L_{\alpha_{i}}^{i}$ annihilates $\mathscr{Q}_{\alpha_{i}}^{i}, 1 \leq i \leq d$, and these operators pairwise commute, $\mathcal{L}_{\alpha}$ is a projection of $\ell_{\infty}$ onto $\mathscr{Q}_{\alpha}$ of norm $\leq \prod_{i=1}^{\alpha}\left(1+\left\|L_{\alpha_{i}}^{i}\right\|\right)=: c(\alpha, d)$.

Now we estimate order of approximation by $\mathcal{L}_{\alpha}$ using mixed $\alpha$-oscillation given for $\alpha \in \mathbb{Z}_{+}^{d}$ and $f \in \ell_{\infty}$ by

$$
\operatorname{osc}_{\alpha} f:=\sup _{x, h}\left\{\left|\left(\prod_{i=1}^{d} \Delta_{h_{i} e^{i}}^{\alpha_{i}} f\right)(x)\right|\right\},
$$

where supremum is taken over $x, h \in \mathbb{R}^{d}$ satisfying the condition

$$
x \in Q^{d}, \quad x_{i}+\alpha_{i} h_{i} \in[0,1], 1 \leq i \leq d,
$$

and $\left\{e^{i}\right\}_{1 \leq i \leq d}$ is the standard orthonormal basis of $\mathbb{R}^{d}$.

In the sequel, we write

$$
\Delta_{h}^{\alpha}:=\prod_{i=1}^{d} \Delta_{h_{i} e^{i}}^{\alpha_{i}} .
$$

Here $\Delta_{h_{i} e^{i}}^{\alpha_{i}}=1$ if $\alpha_{i}=0$; in particular,

$$
\operatorname{osc}_{\alpha} f=\operatorname{osc}_{k}^{i} f \quad \text { if } \quad \alpha=k e^{i} .
$$

Lemma 6.5. It is true that

$$
\left\|f-\mathcal{L}_{\alpha} f\right\|_{\infty} \leq c(\alpha, d) \operatorname{osc}_{\alpha} f .
$$

Proof (induction on $d$ ). For $d=1$ the assertion coincides with inequality (6.3).

Now let (6.15) holds for $d-1 \geq 0$. Setting $\hat{x}:=\left(x_{1}, \ldots, x_{d-1}\right), \hat{\alpha}:=\left(\alpha_{1}, \ldots, \alpha_{d-1}\right)$, etc. for $x \in \mathbb{R}^{d}, \alpha \in \mathbb{Z}_{+}^{d}$ and $\hat{Q}:=[0,1]^{d-1}$ we then have for $g \in \ell_{\infty}(\hat{Q})$

$$
\left\|g-\mathcal{L}_{\hat{\alpha}} g\right\|_{\ell_{\infty}(\hat{Q})} \leq c(\hat{\alpha}, d-1) \operatorname{osc}_{\hat{\alpha}} g .
$$


Now, for $f \in \ell_{\infty}\left(Q^{d}\right), \alpha \in \mathbb{Z}_{+}^{d}$, we have from (6.10)

$$
f-\mathcal{L}_{\alpha} f=\left(\prod_{i=1}^{d-1}\left(1-L_{\alpha_{i}}^{i}\right)\right)\left(f-L_{\alpha_{d}}^{d} f\right)=:\left(1-\mathcal{L}_{\hat{\alpha}}\right) \varphi_{x_{d}}
$$

where $\varphi_{x_{d}}: \hat{x} \mapsto\left(f-L_{\alpha_{d}}^{d} f\right)\left(\hat{x}, x_{d}\right), \hat{x} \in \hat{Q}^{d}$ and $x_{d} \in[0,1]$ is fixed.

Taking here the $\ell_{\infty}(\hat{Q})$-norm and applying ([6.16) we obtain that

$$
\left\|f-\mathcal{L}_{\alpha} f\right\|_{\ell_{\infty}(\hat{Q})} \leq c(\hat{\alpha}, d-1) \operatorname{osc}_{\hat{\alpha}}\left(\varphi_{x_{d}}\right)=c(\hat{\alpha}, d-1) \sup _{\hat{x}, \hat{h}}\left\{\left|\left(\Delta_{\hat{h}}^{\hat{\alpha}}\left(1-L_{\alpha_{d}}^{d}\right) f\right)(x)\right|\right\}
$$

where $\hat{x}, \hat{h}$ satisfy (6.12) for $d-1$ (instead of $d)$.

Further, denoting the function $x_{d} \mapsto \Delta_{\hat{h}}^{\hat{\alpha}} f(x), 0 \leq x_{d} \leq 1$, with fixed $\hat{x}, \hat{h}$ by $\psi_{\hat{x}, \hat{h}}$, changing the order of $\Delta_{\hat{h}}^{\hat{\alpha}}$ and $1-L_{\alpha_{d}}^{d}$ and taking supremum over $0 \leq x_{d} \leq 1$ we obtain from (6.17)

$$
\left\|f-\mathcal{L}_{\alpha} f\right\|_{\infty} \leq c(\hat{\alpha}, d-1) \sup _{\hat{x}, \hat{h}}\left\{\left\|\psi_{\hat{x}, \hat{h}}-L_{\alpha_{d}} \psi_{\hat{x}, \hat{h}}\right\|_{\ell_{\infty}[0,1]}\right\}
$$

Estimating the right-hand side by (6.3) we finally have

$$
\left\|f-\mathcal{L}_{\alpha} f\right\|_{\infty} \leq c(\hat{\alpha}, d-1) c\left(\alpha_{d}\right) \sup _{x, h}\left\{\left|\Delta_{\hat{h}}^{\hat{\alpha}} \Delta_{h_{d} e^{d}}^{\alpha_{d}} f\right|\right\}=: c(\alpha, d) \operatorname{osc}_{\alpha} f .
$$

Now let

$$
\mathcal{L}_{k}:=\prod_{|\alpha|=k} \mathcal{L}_{\alpha}=\prod_{i=1}^{N} \mathcal{L}_{\alpha^{i}}
$$

where $N$ is the cardinality of the set $\left\{\alpha \in \mathbb{Z}_{+}^{d}:|\alpha|=k\right\}$ and $\left\{\alpha^{i}\right\}_{1 \leq i \leq N}$ is its arbitrary enumeration.

Lemma 6.6. It is true that

$$
\left\|f-\mathcal{L}_{k} f\right\|_{\infty} \leq c(k, d) \sum_{|\alpha|=k} \operatorname{osc}_{\alpha} f .
$$

Proof. Using the identity

$$
1-\mathcal{L}_{k}=\sum_{i=1}^{N}\left(\prod_{j=1}^{i-1} \mathcal{L}_{\alpha^{j}}\right)\left(1-\mathcal{L}_{\alpha^{i}}\right)
$$

where $\prod_{j=1}^{i-1}:=1$ for $i=1$ and applying Lemma 6.5 we have

$$
\left\|f-\mathcal{L}_{k} f\right\|_{\infty} \leq\left(\prod_{i=1}^{n-1}\left\|\mathcal{L}_{\alpha^{i}}\right\|\right) \sum_{|\alpha|=k} c(\alpha, d) \operatorname{osc}_{\alpha} f \leq c(k, d) \sum_{|\alpha|=k} \operatorname{osc}_{\alpha} f .
$$


Lemma 6.7. $E_{k}(f) \leq\left\|f-\mathcal{L}_{k} f\right\|_{\infty}$.

Proof. It suffices to prove that $\mathcal{L}_{k} f$ is a polynomial of degree $k-1$.

To this end we note that $\mathcal{L}_{\alpha} f=f$ if

$$
\operatorname{osc}_{\alpha} f=0 \text { for all }|\alpha|=k,
$$

see (6.19).

Since $\operatorname{osc}_{k}^{i} f=\operatorname{osc}_{k e^{i}} f$, see (6.14), and the latter satisfies (6.20) for all $1 \leq i \leq d$, Corollary 6.4 implies that $f$ is a polynomial of degree $k-1$ in each variable.

Due to (6.20) for every $|\alpha|=k$ the mixed difference $\Delta_{h}^{\alpha} f=0$. Dividing by $h^{\alpha}$ and sending $h$ to 0 we obtain that $D^{\alpha} f=0,|\alpha|=k$. Since for every monomial $x^{\beta}$ with $|\beta| \geq k$ there is $|\alpha|=k$ such that $D^{\alpha}\left(x^{\beta}\right) \neq 0$, the polynomial $f$, hence, $\mathcal{L}_{\alpha} f$, contains in its decomposition only monomials $x^{\beta}$ with $|\beta| \leq k-1$.

Hence, $\mathcal{L}_{\alpha} f \in \mathcal{P}_{k-1}^{d}$.

It remains to use Corollary E.4 of [BBI-11, Ch. 2, App.E] which, in particular, implies that

$$
\operatorname{osc}_{\alpha} f \leq c(k, d) \operatorname{osc}_{k}, \quad|\alpha|=k .
$$

The corollary is formulated for continuous $f$ but its proof is based only on the combinatorial identity relating $\Delta_{h}^{\alpha}$ with a linear combination of shifted $k$-differences, see Theorem E.1 there. Hence, inequalities (6.21) hold for $f \in \ell_{\infty}$ as well.

Finally, we combine Lemmas 6.6, 6.7 and inequalities (6.21) to obtain the required inequality

see (6.2).

$$
E_{k} f \leq c(k, d) \operatorname{osc}_{k} f
$$

The proof of the converse inequality is essentially simpler. In fact, since by the definition, see (1.4), $\operatorname{osc}_{k} f \leq 2^{k}\|f\|_{\infty}$ and $\left.\operatorname{osc}_{k}\right|_{\mathcal{P}_{k-1}^{d}}=0$, for every polynomial $m \in \mathcal{P}_{k-1}^{d}$

$$
\operatorname{osc}_{k} f \leq \operatorname{osc}_{k}(f-m) \leq 2^{k}\|f-m\|_{\infty} .
$$

Taking here infimum in $m$ we get

$$
2^{-k} \operatorname{osc}_{k} f \leq E_{k} f
$$

The proof of Theorem 6.1 is complete.

Now Theorem 2.8 follows directly from Theorem 6.1 and the definition of $|\cdot|_{V_{p}^{k}}$, see (1.20).

\section{Proof of Theorem 2.11}

(a) Let $f \in \dot{V}_{p}^{k}$ and $s:=\frac{d}{p} \in(0, k)$. Given $\varepsilon>0$ we should find a function $f_{\varepsilon} \in \Lambda^{k, s}$ such that

$$
\left|\left\{x \in Q^{d}: f(x) \neq f_{\varepsilon}(x)\right\}\right|<\varepsilon .
$$

To this end we first find a set, say, $S_{f} \subset Q^{d}$ of measure 1 such that $f$ is locally Lipschitz at every its point. Then we find a subset of $S_{f}$ denoted by $S_{\varepsilon}$ such that its Lebesgue measure 
is at least $1-\varepsilon$ and the trace $\left.f\right|_{S_{\varepsilon}}$ satisfies the conditions of the extension theorem for Lipschitz functions. Extending $\left.f\right|_{S_{\varepsilon}}$ to a function of $\Lambda^{k, s}$ we finally obtain the required $f_{\varepsilon}$ of (7.1).

We begin with a result on the structure of $E_{k}(f)$ as a function of $Q \subset Q^{d}$. Parameterizing the set of cubes in $\mathbb{R}^{d}$ by the bijection $\mathbb{R}^{d} \times \mathbb{R}_{+} \ni(x, r) \leftrightarrow Q_{r}(x) \subset \mathbb{R}^{d}$, where $Q_{r}(x)$ is a closed cube of sidelength $2 r>0$ centered at $x$, we consider $E_{k}(f)$ as a function of $x, r$. It is easily seen that the subset $\Omega_{0} \subset \mathbb{R}^{d} \times \mathbb{R}_{+}$of cubes $Q_{r}(x) \subset Q^{d}$ is a convex body.

Proposition 7.1. Let $f \in \dot{V}_{p}^{k}, s \in(0, k]$. There is a function $\mathcal{E}_{k}(f): \Omega_{0} \rightarrow \mathbb{R}_{+}$such that the following is true.

(a) $\mathcal{E}_{k}(f)$ is Lebesgue measurable in $x \in Q^{d}$.

(b) For every $Q \subset Q^{d}$

$$
E_{k}(f ; Q) \leq \mathcal{E}_{k}(f ; Q)
$$

(c) For every packing $\pi \in \Pi$

$$
\left\{\sum_{Q \in \pi} \mathcal{E}_{k}(f ; Q)^{p}\right\}^{\frac{1}{p}} \leq 5|f|_{V_{p}^{k}} .
$$

Proof. Let $\left\{f_{n}\right\}_{n \in \mathbb{N}} \subset C^{\infty}$ be a sequence pointwise converging on $Q^{d}$ to $f$ such that

$$
\varlimsup_{n \rightarrow \infty}\left|f_{n}\right|_{V_{p}^{k}} \leq 5|f|_{V_{p}^{k}}, \quad \varlimsup_{n \rightarrow \infty}\left\|f_{n}\right\|_{\infty} \leq 3\|f\|_{\infty}
$$

see Theorem 2.3 .

Then we define the required function by setting for $Q \subset Q^{d}$

$$
\mathcal{E}_{k}(f ; Q):=\varliminf_{n \rightarrow \infty} E_{k}\left(f_{n} ; Q\right)
$$

and prove that $\mathcal{E}_{k}(f)$ satisfies the declared properties.

(a) First we show that $E_{k}(g)$ is continuous in $x \in Q^{d}$ if $g$ is.

Let $Q \subset Q^{d}$ and $h \in \mathbb{R}^{d}$ be such that $Q+h \subset Q^{d}$. Then we have

$$
\begin{aligned}
\left|E_{k}(g ; Q)-E_{k}(g ; Q+h)\right| & =\left|E_{k}(g ; Q)-E_{k}(g(\cdot-h) ; Q)\right| \leq E_{k}(g-g(\cdot-h) ; Q) \\
& \leq \max _{Q}|g(x)-g(x-h)| \rightarrow 0 \quad \text { as } \quad h \rightarrow 0 .
\end{aligned}
$$

In other words,

at every $x$ from the set

$$
\lim _{h \rightarrow 0} E_{k}(g ; x+h, r)=E_{k}(g ; x, r)
$$

$$
\mathcal{Q}_{r}:=\left\{x \in Q^{d}: Q_{r}(x) \subset Q^{d}\right\},
$$

i.e., $E_{k}(g)$ is continuous on the compact convex set $\mathcal{Q}_{r}$.

Now since each $f_{n} \in C^{\infty}$ approximating $f$ is continuous on $Q^{d}$, the function $E_{k}\left(f_{n} ; \cdot ; r\right)$ is continuous in $x$, hence, $\mathcal{E}_{k}(f ; \cdot ; r)$ is Lebesgue measurable in $x \in \mathcal{Q}_{r}$, see (7.5). Therefore it is Lebesgue measurable in $x$ on the set $\cup_{r>0} \mathcal{Q}_{r}=\stackrel{\circ}{Q}^{d}$. 
(b) To prove that $E_{k}(f ; Q) \leq \mathcal{E}_{k}(f ; Q), Q \subset Q^{d}$, we for every $n \in \mathbb{N}$ choose a polynomial $P_{n} \in \mathcal{P}_{k-1}^{d}$ such that

We have

$$
E_{k}\left(f_{n} ; Q\right)=\left\|f_{n}-P_{n}\right\|_{\ell_{\infty}(Q)}
$$

and then by (7.4)

$$
\left\|P_{n}\right\|_{\ell_{\infty}(Q)} \leq\left\|f_{n}\right\|_{\infty}+E_{k}\left(f_{n}, Q\right) \leq 2\left\|f_{n}\right\|_{\infty}
$$

$$
\varlimsup_{n \rightarrow \infty}\left\|P_{n}\right\|_{\ell_{\infty}(Q)} \leq 2 \varlimsup_{n \rightarrow \infty}\left\|f_{n}\right\|_{\infty} \leq 6\|f\|_{\infty}
$$

Hence, $\left\{P_{n}\right\}_{n \in \mathbb{N}}$ is bounded in $\ell_{\infty}(Q)$ and so contains a subsequence $\left\{P_{n_{i}}\right\}_{i \in \mathbb{N}}$ uniformly converging on $Q$ to some $P \in \mathcal{P}_{k-1}^{d}$ such that

$$
\lim _{i \rightarrow \infty}\left\|f-P_{n_{i}}\right\|_{\ell_{\infty}(Q)}=\varliminf_{n \rightarrow \infty}\left\|f_{n}-P_{n}\right\|_{\ell_{\infty}(Q)} .
$$

This then implies that

$$
E_{k}(f ; Q) \leq\|f-P\|_{\ell_{\infty}(Q)} \leq \lim _{i \rightarrow \infty}\left\|f_{n_{i}}-P_{n_{i}}\right\|_{\ell_{\infty}(Q)}=\varliminf_{n \rightarrow \infty}\left\|f_{n}-P_{n}\right\|_{\ell_{\infty}(Q)}=: \mathcal{E}_{k}(f ; Q) .
$$

This proves (17.2) and property (b).

(c) To prove (7.3) we write

$$
\sum_{Q \in \pi} \mathcal{E}_{k}(f ; Q)^{p}:=\sum_{Q \in \pi} \underline{\varliminf_{n \rightarrow \infty}} E_{k}\left(f_{n} ; Q\right)^{p} \leq \varliminf_{n \rightarrow \infty} \sum_{Q \in \pi} E_{k}\left(f_{n} ; Q\right)^{p} \leq \varliminf_{n \rightarrow \infty}\left|f_{n}\right|_{V_{p}^{k}}^{p} \leq\left(5|f|_{V_{p}^{k}}\right)^{p} .
$$

This proves (7.3) and the proposition.

Proposition 7.2. Under the assumptions of Proposition 7.1

$$
\varlimsup_{Q \rightarrow x}\left(|Q|^{-\frac{1}{p}} E_{k}(f ; Q)\right)<\infty
$$

for almost all $x \in Q^{d}$.

Proof. Let $\rho_{a}: \mathcal{Q}_{a} \rightarrow \mathbb{R}_{+}, a>0$, be a function given by

$$
\rho_{a}(x):=\sup \left\{r^{-\frac{d}{p}} \mathcal{E}_{k}(f ; x, r): r \leq a\right\} ;
$$

here $\mathcal{Q}_{a}$ is the subset of $Q^{d}$ given by (7.6).

Given $N \in \mathbb{N}$ we then set

$$
S_{a, N}:=\left\{x \in \mathcal{Q}_{a}: \rho_{a}(x)>N\right\} .
$$

Lemma 7.3. Let $|f|_{V_{p}^{k}}=1$. Then

$$
\left|S_{a, N}\right| \leq c(d, p) N^{-p} .
$$

Proof. Let $S \subset Q^{d}$ and

$$
M(S):=\inf _{\Delta}\left\{\sum_{Q \in \Delta}|Q|\right\},
$$

where $\Delta$ runs over all coverings of $S$ by subcubes of $Q^{d}$. 
It is known that for a measurable set $S$

$$
|S| \leq M(S)
$$

see, e.g., [Ca-67, Thm. I.1].

Now let $x \in S_{a, N}$. By definitions (7.8), (7.9) there is a cube $Q_{x}$ of radius $\leq a$ centered at $x \in \mathcal{Q}_{a}$ such that

$$
\mathcal{E}_{k}\left(f ; Q_{x}\right)>N\left|Q_{x}\right|^{\frac{1}{p}} .
$$

Since the family $\left\{Q_{x}\right\}$ covers $S_{a, N}$ by centers of its cubes, the Besicovich covering theorem, see, e.g., Gu-75, Thm.1.2] asserts that there is a countable subcovering of $\left\{Q_{x}\right\}$, say $\left\{Q_{i}\right\}_{i \in \mathbb{N}}$, which is the union of at most $c(d)$ packings $\pi_{i} \in \Pi$. This and (7.11)-(17.13) imply that

$$
\left|S_{a, N}\right| \leq \sum_{i \leq c(d)} \sum_{Q \in \pi_{i}}|Q| \leq N^{-p} \sum_{i \leq c(d)} \sum_{Q \in \pi_{i}} \mathcal{E}_{k}(f ; Q)^{p} .
$$

By (7.3) the inner sum in the right-hand side is bounded from above by $\left(5|f|_{V_{p}^{k}}\right)^{p}=5^{p}$, hence,

$$
\left|S_{a, N}\right| \leq 5^{p} c(d) N^{-p}
$$

Now we have from (7.10)

$$
\left|S_{a, \infty}\right|=\left|\bigcap_{N \in \mathbb{N}} S_{a, N}\right|=0
$$

moreover, by monotonicity of sets $S_{a, \infty}$ under inclusions, $S_{\infty}:=\cup_{a>0} S_{a, \infty}$ is of (Lebesgue) measure zero.

Finally, for every $x \in \mathcal{Q}_{a} \backslash S_{a, \infty}$

$$
\sup _{r \leq a} r^{-\frac{d}{p}} \mathcal{E}_{k}(f ; x, r)<\infty \text { and } \bigcup_{0<a \leq \frac{1}{2}} \mathcal{Q}_{a}=\stackrel{Q}{ }^{d},
$$

see (7.6).

Hence, for every point $x \in Q^{d}$ outside set $S_{\infty} \cup \partial Q^{d}$ of measure 0

$$
\varlimsup_{Q \rightarrow x}|Q|^{-\frac{1}{p}} \mathcal{E}_{k}(f ; Q)<\infty
$$

This and (7.2) give (7.7).

Proposition 7.2 is proved.

In the next auxiliary result we use the following:

Definition 7.4. A Lebesgue measurable set $S \subset \mathbb{R}^{d}$ is said to be Ahlfors d-regular (briefly, regular) if for every $x \in S$ and some constants $\delta \in(0,1), r_{0}>0$,

$$
\left|Q_{r}(x) \cap S\right| \geq \delta\left|Q_{r}(x)\right|, \quad 0<r \leq r_{0} .
$$


Lemma 7.5. Let $f \in V_{p}^{k}, s:=\frac{d}{p} \in(0, k]$. Given $\varepsilon \in(0,1)$ there is a regular set, say, $\Sigma_{\varepsilon} \subset Q^{d}$ such that

$$
\left|\Sigma_{\varepsilon}\right| \geq 1-\varepsilon
$$

and, moreover, there are positive constants $c_{\varepsilon}, a_{\varepsilon}$ such that

$$
\sup _{x \in \Sigma_{\varepsilon}} E_{k}(f ; x, r) \leq c_{\varepsilon} r^{s}, \quad 0 \leq r \leq a_{\varepsilon} .
$$

Proof. We set

$$
a_{\varepsilon}:=\frac{1}{2}\left(1-\sqrt[d]{1-\frac{\varepsilon}{3}}\right)
$$

By (7.14) we have for every $x \in \mathcal{Q}_{a_{\varepsilon}} \backslash S_{a_{\varepsilon}, \infty}$ and $r_{n}:=2^{-n} a_{\varepsilon}$

$$
\varphi(x):=\sup _{n \in \mathbb{Z}_{+}}\left\{r_{n}^{-s} \mathcal{E}_{k}\left(f ; x, r_{n}\right)\right\}<\infty .
$$

Since the sequence under supremum is measurable in $x$, see Proposition 7.1(a), $\varphi$ is measurable and finite at every point of $\mathcal{Q}_{a_{\varepsilon}} \backslash S_{a_{\varepsilon}, \infty}$. Hence, there are a set $\Omega_{\varepsilon} \subset \mathcal{Q}_{a_{\varepsilon}} \backslash S_{a, \infty}$ and a constant $N_{\varepsilon}$ such that

$$
\varphi \leq N_{\varepsilon} \quad \text { on } \quad \Omega_{\varepsilon} \quad \text { and } \quad\left|\Omega_{\varepsilon}\right| \geq\left|\mathcal{Q}_{a_{\varepsilon}}\right|-\frac{\varepsilon}{3} .
$$

Further, the Lebesgue density theorem implies that

$$
\lim _{r \rightarrow 0} \frac{\left|\Omega_{\varepsilon} \cap Q_{r}(x)\right|}{\left|Q_{r}(x)\right|}=1 \text { a.e. in } \Omega_{\varepsilon} .
$$

By Egorov's theorem there is a measurable subset $\Sigma_{\varepsilon} \subset \Omega_{\varepsilon}$ of measure

$$
\left|\Sigma_{\varepsilon}\right|>\left|\Omega_{\varepsilon}\right|-\frac{\varepsilon}{3}
$$

such that (7.21) converges uniformly on $\Sigma_{\varepsilon}$. Assuming without loss of generality that all $x \in \Sigma_{\varepsilon}$ are simultaneously density points of $\Omega_{\varepsilon}$ and $\Sigma_{\varepsilon}$ we also have

$$
1=\lim _{r \rightarrow 0} \frac{\left|\Omega_{\varepsilon} \cap Q_{r}(x)\right|}{(2 r)^{d}}=\lim _{r \rightarrow 0} \frac{\left|\Sigma_{\varepsilon} \cap Q_{r}(x)\right|}{(2 r)^{d}}
$$

for every $x \in \Sigma_{\varepsilon}$.

This implies that the second limit also converges on $\Sigma_{\varepsilon}$ uniformly.

Hence, for every $x \in \Sigma_{\varepsilon}$ and some $0<r_{0}<1$ (depending on $\Omega_{\varepsilon}$ )

$$
\frac{\left|\Sigma_{\varepsilon} \cap Q_{r}(x)\right|}{(2 r)^{d}}>\frac{1}{2} \quad \text { for } \quad 0<r \leq r_{0}
$$

i.e., $\Sigma_{\varepsilon}$ is regular.

Let us show that $\Sigma_{\varepsilon}$ satisfy inequality (7.16). In fact, due to (7.6) and (7.18)

$$
\left|\Sigma_{\varepsilon}\right|>\left|\Omega_{\varepsilon}\right|-\frac{\varepsilon}{3}>\left|\mathcal{Q}_{a_{\varepsilon}}\right|-\frac{2 \varepsilon}{3}=\left(1-2 a_{\varepsilon}\right)^{d}-\frac{2 \varepsilon}{3}=1-\varepsilon
$$

as required. 
Finally, $\varphi \leq N_{\varepsilon}$ on the regular set $\Sigma_{\varepsilon}$, hence, for $x \in \Sigma_{\varepsilon}$

$$
\mathcal{E}_{k}\left(f ; x, 2^{-n} a_{\varepsilon}\right) \leq N_{\varepsilon}\left(2^{-n} a_{\varepsilon}\right)^{s}, \quad n \in \mathbb{Z}_{+} .
$$

Enlarging $N_{\varepsilon}$ (and denoting its new value by $c_{\varepsilon}$ ) we can replace here $2^{-n} a_{\varepsilon}$ by an arbitrary $r \in\left(0, a_{\varepsilon}\right]$. Moreover, by Proposition 7.1(b), $E_{k}(f) \leq \mathcal{E}_{k}(f)$, hence, for $x \in \Sigma_{\varepsilon}$

$$
E_{k}(f ; x, r) \leq c_{\varepsilon} r^{s}, \quad 0<r \leq a_{\varepsilon} .
$$

This gives (7.17) and proves the lemma.

Proceeding the proof of Theorem we apply inequality (17.17) to the trace $\left.f\right|_{\Sigma_{\varepsilon}}$. This function satisfies inequality

$$
E_{k}\left(\left.f\right|_{\Sigma_{\varepsilon}} ; Q_{r}(x)\right) \leq c_{\varepsilon} r^{s}, \quad 0<r \leq a_{\varepsilon},
$$

on the regular set $\Sigma_{\varepsilon}$ and therefore meets the conditions of the extension theorem [Br1-70], see [BBII-11, Thm.9.30] for the general version of this result. Due to this theorem there is a function $f_{\varepsilon}: \mathbb{R}^{d} \rightarrow \mathbb{R}$ such that

$$
\left.f_{\varepsilon}\right|_{\Sigma_{\varepsilon}}=\left.f\right|_{\Sigma_{\varepsilon}}
$$

and, moreover,

$$
\sup _{x \in Q^{d}} E_{k}\left(f_{\varepsilon} ; x, r\right) \leq c r^{s}, \quad 0 \leq r \leq 1 ;
$$

here $c$ depends on $\varepsilon, d, k$ and $f$.

Using further Theorem 6.1 we replace $E_{k}\left(f_{\varepsilon} ; x, r\right)$ by $\operatorname{osc}_{k}\left(f ; Q_{r}(x)\right)$ and finally obtain

$$
\left|\Delta_{h}^{k} f_{\varepsilon}(x)\right| \leq \operatorname{osc}_{k}(f ; x, k\|h\|) \leq c\|h\|^{s},
$$

where $\|h\|:=\max _{1 \leq i \leq d}\left|h_{i}\right|$ and $x, x+h$ belong to $Q^{d}$.

By definition, see (2.8), (2.9), this means that $f_{\varepsilon} \in \Lambda^{k, s}, 0<s \leq k$.

Thus, we have proved that for $f \in \dot{V}_{p}^{k}$ with $s \in(0, k]$ and $\varepsilon>0$ there is a Lipschitz function $f_{\varepsilon} \in \Lambda^{k, s}$ such that

$$
f=f_{\varepsilon} \quad \text { outside a set of Lebesgue measure }<\varepsilon .
$$

For $0<s<k$, this establishes assertion (a) of Theorem 2.11

Finally, in part (b) of this theorem we should prove that if $s=k$ we can replace the function $f_{\varepsilon} \in \Lambda^{k, k}$ by a $C^{k}$ function. To this end we use the linear continuous isomorphism

$$
\Lambda^{k, k}=C^{k-1,1} \cong \dot{W}_{\infty}^{k} .
$$

see Proposition 2.10 and Br1-70, respectively.

More precisely, every class $f \in \dot{W}_{\infty}^{1}$ contains a unique $C^{k-1,1}$ function, say $\hat{f}$, such that the map $f \mapsto \hat{f}$ is a linear continuous bijection of $\dot{W}_{\infty}^{k}$ onto $C^{k-1,1}$.

Further, $\dot{W}_{\infty}^{k} \subset \dot{W}_{p}^{k}, p>1$, while for every function $\hat{f}$ from the class $f \in \dot{W}_{p}^{k}$ there is a function $f_{\varepsilon} \in C^{k}$ such that $f$ coincides with $f_{\varepsilon}$ outside a set of measure $<\varepsilon$, see CZ-61, Thm. 4.13]. Combining this with (7.24) we conclude that for every $g \in C^{k-1,1}$ there is $g_{\varepsilon} \in C^{k}$ coinciding with $g$ outside of a set of measure $<\varepsilon$. 
Taking now $f \in \dot{V}_{p}^{k}$ with $s=k$ and $g=f_{\varepsilon}$ from (7.23) we obtain the function $g_{\varepsilon} \in C^{k}$ coinciding with $f$ outside a set of measure $<2 \varepsilon$.

This proves assertion (b) and the theorem.

\section{Proof of Theorem 2.15}

(a) We should prove that if $f \in \dot{V}_{p}^{k}$ and $s:=\frac{d}{p} \in(0, k]$, then

$$
f \in \Lambda^{k, s}(x) \text { a. e. }
$$

To this end we use Proposition 7.2 asserting that for this $f$

$$
\varlimsup_{r \rightarrow 0} r^{-s} E_{k}(f ; x, r)<\infty \text { for all } x \in S_{f},
$$

where $S_{f}$ is a subset of $\AA^{d}$ of measure 1 .

Due to inequality (2.7) $E_{k}(f)$ here can be replaced by $\operatorname{osc}_{k}(f)$; after this change condition (8.2) at $x \in S_{f}$ coincides with the condition of Definition 2.12 introducing the space $\Lambda^{k, s}\left(x_{0}\right)$. Hence, we obtain that $f \in \Lambda^{k, s}(x)$ for all $x \in S_{f} \subset \stackrel{\circ}{Q}^{d}$, i.e., almost everywhere on $Q^{d}$.

(b) We should prove that the function under consideration belongs to the Taylor space $T^{s}(x)$ for almost all $x \in Q^{d}$.

To this end we use Theorem 3 from [Br1-94, §2] that, in particular, asserts the following:

If a function $f \in \ell_{\infty}\left(\stackrel{Q}{ }^{d}\right)$ satisfies at a given point $x_{0}$ the condition

$$
\varlimsup_{r \rightarrow 0} r^{-s} E_{k}(f ; x, r)<\infty,
$$

where $0<s \leq k$, then $f \in T^{s}\left(x_{0}\right)$.

It then follows from here and condition (8.2) that $f \in T^{s}(x)$ for every $x \in S_{f}$, i.e., for almost all $x \in Q^{d}$.

(c) Now we should show that if $f \in \dot{V}_{p}^{k}$ and $s=k$, then $f \in t^{k}\left(x_{0}\right)$ a.e.

To this end we first note that due to the previous result $f \in T^{k}(x)$ for every $x \in S_{f}$, i.e., for every such $x$ there is the Taylor polynomial $T_{x}(f) \in \mathcal{P}_{k-1}^{d}$ and constants $c_{1}, c_{2}>0$ independent of $r$ such that

$$
\max _{Q_{r}(x)}\left|f-T_{x}(f)\right| \leq c_{1} r^{k} \quad \text { for } \quad 0<r \leq c_{2} .
$$

Further, we use Theorem 2.11 asserting that given $\varepsilon>0$ there is a regular subset $S_{\varepsilon} \subset \grave{Q}^{d}$ and a function $f_{\varepsilon} \in C^{k}$ such that

$$
\left|S_{\varepsilon}\right|>1-\varepsilon \quad \text { and } \quad f=f_{\varepsilon} \quad \text { on } \quad S_{\varepsilon} .
$$

Now we derive from here and (8.3) that

$$
f \in t^{k}(x) \text { for almost all } x \in S_{\varepsilon} .
$$

Since $\left|\cup_{\varepsilon>0} S_{\varepsilon}\right|=1$, this implies that $f \in t^{k}(x)$ for almost all $x$ in $Q^{d}$. 
Let $T_{x}\left(f_{\varepsilon}\right) \in \mathcal{P}_{k}^{d}$ be the Taylor polynomial of $f_{\varepsilon}$ at $x$, i.e.,

$$
\max _{Q_{r}(x)}\left|f_{\varepsilon}-T_{x}\left(f_{\varepsilon}\right)\right| \leq c(r) r^{k}, \quad 0<r \leq \frac{1}{2}
$$

where $c(r) \rightarrow 0$ as $r \rightarrow 0$.

We write

$$
T_{x}\left(f_{\varepsilon}\right):=\hat{T}_{x}\left(f_{\varepsilon}\right)+\sum_{|\alpha|=k} c_{\alpha}\left(f_{\varepsilon}\right)\left(\cdot-x_{0}\right)^{\alpha},
$$

where $\hat{T}_{x}\left(f_{\varepsilon}\right)$ is the Taylor polynomial for $f_{\varepsilon}$ at $x$ of degree $k-1$. In particular,

$$
\max _{Q_{r}(x)}\left|f_{\varepsilon}-\hat{T}_{x}\left(f_{\varepsilon}\right)\right| \leq c_{1} r^{k} \quad \text { for } \quad 0<r \leq \frac{1}{2}
$$

with a constant independent of $r$.

Lemma 8.1. If $x \in S_{\varepsilon} \cap S_{f}$, then

$$
T_{x}(f)=\hat{T}_{x}\left(f_{\varepsilon}\right) .
$$

Proof. By regularity of $S_{\varepsilon}$ there are positive constants $\gamma, r_{0}$ such that for every $x \in S_{\varepsilon}$

$$
\left|S_{\varepsilon} \cap Q_{r}(x)\right| \geq \gamma\left|Q_{r}(x)\right| \text { for } 0<r \leq r_{0} .
$$

Moreover, $f=f_{\varepsilon}$ on $S_{\varepsilon} \cap Q_{r}(x)$; hence,

$$
\max _{S_{\varepsilon} \cap Q_{r}(x)}\left|T_{x}(f)-\hat{T}_{x}\left(f_{\varepsilon}\right)\right| \leq \max _{Q_{r}(x)}\left|f-T_{x}(f)\right|+\max _{Q_{r}(x)}\left|f_{\varepsilon}-\hat{T}_{x}\left(f_{\varepsilon}\right)\right| .
$$

Estimating the right-hand side by inequalities (8.3) and (8.7) we have for every $x \in S_{\varepsilon} \cap S_{f}$

$$
\max _{S_{\varepsilon} \cap Q_{r}(x)}\left|T_{x}(f)-\hat{T}_{x}\left(f_{\varepsilon}\right)\right| \leq c_{3} r^{k}, \quad 0 \leq r \leq c_{2}
$$

for some $c_{3}>0$ independent of $r$.

Further, using a Remez type inequality [BG-73] and (8.9) we can estimate the left-hand side of (8.10) from below by $c(k, d) \gamma^{k-1} \max _{Q_{r}(x)}\left|T_{x}(f)-\hat{T}_{x}\left(f_{\varepsilon}\right)\right|$.

This implies for sufficiently small $r>0$ the inequality

$$
\max _{Q_{r}(x)}\left|T_{x}(f)-\hat{T}_{x}\left(f_{\varepsilon}\right)\right| \leq c r^{k}
$$

with $c$ independent of $r$.

Dividing this by $r^{k}$ and letting $r$ to 0 we conclude that every coefficient of this polynomial (of degree $k-1$ ) should be 0 .

Finally, we show that $f \in t^{k}(x)$ for almost all $x \in S_{\varepsilon}$.

In fact, for the function $F:=f-f_{\varepsilon}$ and $x \in S_{\varepsilon} \cap S_{f}$ we have by Lemma 8.1 and inequalities (8.3), (8.7)

$$
\max _{Q_{r}(x)}|F| \leq \max _{Q_{r}(x)}\left|f_{\varepsilon}-\hat{T}_{x}\left(f_{\varepsilon}\right)\right|+\max _{Q_{r}(x)}\left|f-T_{x}(f)\right| \leq c_{1} r^{k}, \quad 0<r \leq c_{2},
$$

where $c_{1}, c_{2}>0$ are independent of $r$. 
Inequality (8.11) and Theorem 10 from [CZ-61] imply that for almost all $x \in S_{\varepsilon} \cap S_{f}$

$$
\max _{Q_{r}(x)}|F|=o\left(r^{k}\right), \quad r \rightarrow 0 .
$$

In turn, for such $x$ we derive from here and (8.6)

$$
\max _{Q_{r}(x)}\left|f-T_{x}\left(f_{\varepsilon}\right)\right| \leq \max _{Q_{r}(x)}|F|+\max _{Q_{r}(x)}\left|f_{\varepsilon}-T_{x}\left(f_{\varepsilon}\right)\right|=o\left(r^{k}\right), \quad r \rightarrow 0 .
$$

Since $\left|S_{\varepsilon} \cap S_{f}\right|=\left|S_{\varepsilon}\right|, f \in t^{k}(x)$ for almost all $x \in S_{\varepsilon}$.

This proves assertion (c) of Theorem 2.15 .

\section{Proofs of Theorem 2.18 and Corollary 2.20}

Proof of Theorem 2.18. (a) We should show that the map $L: f \mapsto \tilde{f}$ sending a function $f \in N \dot{V}_{p}^{k}$ to its equivalence class $\tilde{f} \in L_{p}$ is a linear continuous injection of $N \dot{V}_{p}^{k}$ in $\Lambda_{p \infty}^{k s}$, $0<s \leq k$.

In fact, the injectivity of the map follows from the definition of the class $N \dot{V}_{p}^{k}$, see Corollary 2.2, and so it remains to show that its image belongs to $\Lambda_{p \infty}^{k s}$ and the map acts to this space continuously.

To this end, we again apply Theorem 4 from [Br1-71, §2] to write

$$
t^{-s} \omega_{k p}(\tilde{f} ; t) \leq c t^{-s}\left(\sum_{Q \in \pi} E_{k p}(\tilde{f} ; Q)^{p}\right)^{\frac{1}{p}}
$$

where $\pi$ is some packing in $\Pi\left(Q^{d}\right)$ containing cubes of volume $\leq t^{d}$.

For these cubes, we clearly have

$$
E_{k p}(\tilde{f} ; Q) \leq|Q|^{\frac{1}{p}} E_{k \infty}(\tilde{f} ; Q) \leq c t^{\frac{d}{p}} E_{k}(f ; Q):=c t^{s} E_{k}(f ; Q) .
$$

Together with the previous inequality this implies

$$
t^{-s} \omega_{k p}(\tilde{f} ; t) \leq c\left(\sum_{Q \in \pi} E_{k}(f ; Q)^{p}\right)^{\frac{1}{p}} .
$$

Taking here supremums over $t>0$ and all $\pi \in \Pi$, we finally have

$$
|\tilde{f}|_{\Lambda_{p \infty}^{k s}}:=\sup _{t>0} t^{-s} \omega_{k p}(\tilde{f} ; t) \leq c \sup _{\pi}\left(\sum_{Q \in \pi} E_{k}(f ; Q)^{p}\right)^{\frac{1}{p}}=: c|f|_{V_{p}^{k}} .
$$

Hence, the map under consideration acts continuously from $N \dot{V}_{p}^{k}$ in $\Lambda_{p \infty}^{k s}$, i.e., gives the required implication $N \dot{V}_{p}^{k} \hookrightarrow \Lambda_{p \infty}^{k s}$, see (2.24).

Further, let the space $\dot{V}_{p}^{k}$ be such that

$$
s:=\frac{d}{p} \in(0, k) .
$$


We will show that every class $f \in \Lambda_{p 1}^{k s}$ contains a unique representative $\hat{f} \in C$ and the map $f \mapsto \hat{f}$ is a linear continuous injection of $\Lambda_{p 1}^{k s}$ into $\dot{V}_{p}^{k}$. This clearly proves the required implication

$$
\Lambda_{p 1}^{k s} \hookrightarrow \dot{V}_{p}^{k}
$$

This will complete the proof of part (a) of Theorem 2.18.

To prove (9.2) we given $f \in L_{p}$ denote by $\mathcal{L}_{f}$ the Lebesgue set of $f$, i.e., $Q^{d} \backslash \mathcal{L}_{f}$ is of measure zero and the limit over cubes $Q$ centered at $x$

$$
\hat{f}(x):=\lim _{Q \rightarrow x} \frac{1}{|Q|} \int_{Q} f d y
$$

exists at every $x \in \mathcal{L}_{f}$.

Lemma 9.1. If $f \in L_{p}$ is such that

$$
\int_{0}^{1} \frac{\omega_{k p}(f ; t)}{t^{s+1}} d t<\infty
$$

for $0<s<k$, then $\hat{f}$ is uniformly continuous on $\mathcal{L}_{f}$ and for every subcube $Q \subset Q^{d}$

$$
E_{k \infty}(f ; Q) \leq c(k, d) \int_{0}^{|Q|^{\frac{1}{d}}} \frac{\omega_{k p}(f ; t ; Q)}{t^{s+1}} d t .
$$

Hereafter, we as above set

$$
E_{k p}(f ; Q):=\inf \left\{\|f-m\|_{L_{p}(Q)}: m \in \mathcal{P}_{k-1}^{d}\right\}
$$

and denote by $m_{Q}(f)$ an optimal polynomial for this relation.

Proof. Setting for brevity

$$
\omega(t):=\omega_{k p}(f ; t ; Q), \quad t>0,
$$

we estimate the nonincreasing rearrangement $\left(f-m_{Q}(f)\right)^{*}$ by

$$
\left(f-m_{Q}(f)\right)^{*}(t) \leq c(k, d) \int_{\frac{t}{2}}^{|Q|} \frac{\omega\left(u^{\frac{1}{d}}\right)}{u^{1+\frac{1}{p}}} d u, \quad 0 \leq t \leq|Q|,
$$

see [Br1-94, App. II, Cor. 2'].

Sending here $t$ to 0 and noting that $s=\frac{d}{p}$ we then obtain

$$
E_{k \infty}(f ; Q) \leq\left\|f-m_{Q}(f)\right\|_{L_{\infty}(Q)}=\lim _{t \rightarrow \infty}\left(f-m_{Q}(f)\right)^{*}(t) \leq c(k, d) \int_{0}^{|Q|^{\frac{1}{d}}} \frac{\omega(u)}{u^{s+1}} d u .
$$

This proves (9.5).

Now we show that the function $\hat{f}: \mathcal{L}_{f} \rightarrow \mathbb{R}$ is uniformly continuous.

In fact, by (9.5)

$$
\lim _{Q \rightarrow x} E_{k \infty}(f ; Q)=0
$$


and the convergence is uniform in $x$. As in the proof of Theorem 2.6 we derive from here that

$$
\lim _{t \rightarrow 0} \omega_{1 \infty}(f ; t)=0 .
$$

Finally, let $x, x+h \in \mathcal{L}_{f}\left(\subset \stackrel{\circ}{Q}^{d}\right)$. Then

$$
|\hat{f}(x+h)-\hat{f}(x)| \leq \varlimsup_{Q \rightarrow x} \frac{1}{|Q|} \int_{Q}|f(y+h)-f(y)| d y \leq \omega_{1 \infty}(f ;\|h\|) \rightarrow 0 \quad \text { as } \quad h \rightarrow 0 .
$$

Hence, $\hat{f}$ is uniformly continuous on the dense in $Q^{d}$ set $\mathcal{L}_{f}$ and so can be continuously extended to $Q^{d}$; we preserve the same notation $\hat{f}$ for the extension.

Lemma 9.1 is proved.

Now let us check that the regularization $\hat{f}$, see (9.3), satisfies

$$
E_{k \infty}(f ; Q)=E_{k}(\hat{f} ; Q) \text { for all } Q \subset Q^{d} .
$$

Indeed, let $m_{Q} \in \mathcal{P}_{k-1}^{d}$ be such that

$$
E_{k \infty}(f ; Q)=\left\|f-m_{Q}\right\|_{L_{\infty}(Q)} .
$$

Given $\varepsilon \in(0,1)$ we choose $\hat{f}_{\varepsilon} \in \ell_{\infty}$ of the class $f \in L_{\infty}$ such that

$$
\sup _{Q}\left|\hat{f}_{\varepsilon}-m_{Q}\right| \leq\left\|f-m_{Q}\right\|_{L_{\infty}(Q)}+\varepsilon .
$$

Since $\hat{f}$ and $\hat{f}_{\varepsilon}$ coincide on a set of complete Lebesgue measure, say $S_{\varepsilon}$, we have

$$
\hat{f}-m_{Q}=\hat{f}_{\varepsilon}-m_{Q} \quad \text { on } \quad S_{\varepsilon} \cap \mathcal{L}_{f} .
$$

This, in turn, implies

$$
E_{k}(\hat{f} ; Q) \leq \sup _{Q}\left|\hat{f}-m_{Q}\right|-\sup _{S_{\varepsilon} \cap \mathcal{L}_{f}}\left|\hat{f}-m_{Q}\right| \leq \sup _{Q}\left|\hat{f}_{\varepsilon}-m_{Q}\right| \leq\left\|f-m_{Q}\right\|_{L_{\infty}(Q)}+\varepsilon=E_{k \infty}(f ; Q)+\varepsilon .
$$

Hence,

$$
E_{k}(\hat{f} ; Q) \leq E_{k \infty}(f ; Q) .
$$

Since the converse inequality is evident, (9.10) is proved.

Now let $\pi \in \Pi$ be a packing. Due to Lemma 9.1, equality (9.10) and Minkowski inequality we have

$$
\begin{aligned}
\left(\sum_{Q \in \pi} E_{k}(\hat{f} ; Q)^{p}\right)^{\frac{1}{p}} & \leq c\left(\sum_{Q \in \pi}\left(\int_{0}^{|Q|^{\frac{1}{d}}} \frac{\omega_{k p}(f ; t ; Q)}{t^{s+1}} d t\right)^{p}\right)^{\frac{1}{p}} \\
& \leq c \int_{0}^{1} t^{-s-1}\left(\sum_{Q \in \pi} \omega_{k p}(f ; t ; Q)^{p}\right)^{\frac{1}{p}} d t .
\end{aligned}
$$

Hereafter all constants depend only on $k, d$. 
Lemma 9.2. It is true that

$$
\left(\sum_{Q \in \pi} \omega_{k p}(f ; t ; Q)^{p}\right)^{\frac{1}{p}} \leq c \omega_{k p}(f ; t)
$$

Proof. By Theorem 4 from [Br1-71, §2]

$$
\omega_{k p}(f ; t ; Q) \approx \sup _{\pi \in \Pi_{t}(Q)}\left(\sum_{R \in \pi} E_{k p}(f ; R)^{p}\right)^{\frac{1}{p}},
$$

where $\Pi_{t}(Q)$ consists of all packings in $Q$ with cubes of volume $\leq t^{d}$.

Hence, for every $Q$ there is a packing, say $\pi_{Q} \in \Pi_{t}(Q)$, such that

$$
\omega_{k p}(f ; t ; Q) \leq c\left(\sum_{R \in \pi_{Q}} E_{k p}(f ; R)^{p}\right)^{\frac{1}{p}} \text {. }
$$

Therefore the left-hand side of (19.12) is bounded by $c\left(\sum_{Q \in \pi} \sum_{R \in \pi_{Q}} E_{k p}(f ; R)^{p}\right)^{\frac{1}{p}}$.

Since the set of subcubes $\cup_{Q \in \pi} \pi_{Q}$ is a packing in $Q^{d}$ containing only cubes of volume $\leq t^{d}$, the equivalence (9.13) bounds the above expression by $c \omega_{k p}(f ; t)\left(=c \omega_{k p}\left(f ; t ; Q^{d}\right)\right)$.

This proves (9.12).

Now combining (9.11) and (9.12) and taking supremum over $\pi \in \Pi$ we finally have

$$
|\hat{f}|_{V_{p}^{k}}:=\sup _{\pi}\left(\sum_{Q \in \pi} E_{k}(\hat{f} ; Q)^{p}\right)^{\frac{1}{p}} \leq c \int_{0}^{1} \frac{\omega_{k p}(f ; t)}{t^{s+1}} d t=: c|f|_{\Lambda_{p 1}^{k s}} .
$$

This proves that the linear injection $f \mapsto \hat{f}$ maps $\Lambda_{p 1}^{k s}$ in $\dot{V}_{p}^{k} \cap C \subset N \dot{V}_{p}^{k}$ and is bounded, i.e., implication (9.2) holds.

Theorem 2.18 (a) is proved.

(b) Now we should prove that if $k=s=d$, then the following linear isomorphism is given by the operator $\left.L\right|_{N \dot{V}_{p}^{k}}$

$$
\operatorname{Lip}_{1}^{d} \cong N \dot{V}_{1}^{d}
$$

Since the injection $\left.L\right|_{N \dot{V}_{p}^{k}}: N \dot{V}_{p}^{k} \rightarrow \Lambda_{p \infty}^{k s}, 0<s \leq k$, of part (a) of the theorem implies for $k=s=d$ the injection $\left.L\right|_{N \dot{V}_{1}^{d}}: N \dot{V}_{1}^{d} \rightarrow L i p_{1}^{d}$, it remains to prove that it is onto. In the derivation, we need the following Sobolev type embedding that is interesting in its own right result.

Proposition 9.3. It is true that

$$
\operatorname{Lip}_{1}^{d} \subset \dot{V}_{1 \infty}^{d}
$$


Proof. In the proof, we use the following known results.

Let $B V^{k}(Q)$ be a linear space of $L_{1}$ functions on $Q$ whose $k$-th distributional derivatives are finite Borel measures. It is endowed by a seminorm given for $f \in L_{1}(Q)$ by

$$
|f|_{B V^{k}}:=\max _{|\alpha|=k}\left\{\operatorname{var} D^{\alpha} f\right\} .
$$

Theorem A ([Br1-70] Theorem 4). It is true that

$$
\operatorname{Lip}_{1}^{k}(Q)=B V^{k}(Q)
$$

with equivalence constants of seminorms depending only on $k$ and $d$.

The second result is the embedding theorem for Sobolev space $W_{1}^{d}:=W_{1}^{d}\left(Q^{d}\right)$.

Theorem B ([Ga-59]). It is true that

$$
W_{1}^{d} \hookrightarrow C,
$$

where the embedding constant depends only on $d$.

As a consequence we have the following:

Lemma 9.4. For each function $f \in B V^{d}(Q), Q \subset Q^{d}$, it is true that $f \in L_{\infty}$ and

$$
E_{d \infty}(f ; Q) \leq c(d)|f|_{B V^{d}(Q)} .
$$

Proof. We begin with some facts of interpolation space theory.

First, we recall that the $K$-functional of an embedded pair $X \subset Y$ of Banach spaces is a function on $Y \times \mathbb{R}_{+}$given for $y \in Y, t \in \mathbb{R}_{+}$by

$$
K(t ; y ; X, Y):=\inf _{y=x+z}\left\{\|x\|_{X}+t\|z\|_{Y}\right\} .
$$

Second, the relative (Gagliardo) completion of $X$ in $Y$ denoted by $X^{c, Y}$ is a Banach space whose closed unit ball is the closure of that of $X$ in $Y$.

The relation between these notions is given by the following:

Proposition 9.5 ([BK-91], Prop. 2.2.20). It is true that

$$
\|y\|_{X^{c, Y}}=\sup _{t>0} t^{-1} K(t ; y ; X, Y) .
$$

Further, we use the following well-known result, see, e.g., [JH-77] and references therein,

$$
K\left(t ; f ; L_{p} ; W_{p}^{k}\right) \approx t\|f\|_{p}+\omega_{k p}\left(f ; t^{\frac{1}{k}}\right), \quad t>0,
$$

with constants of equivalence depending only on $k$ and $d$.

Along with (9.20) and Theorem A this implies

$$
\|f\|_{\left(W_{1}^{k}\right)^{c, L_{1}}} \approx\|f\|_{1}+\sup _{t>0} \frac{\omega_{k 1}(f ; t)}{t^{k}}:=\|f\|_{1}+|f|_{L i p_{1}^{k}} \approx\|f\|_{1}+|f|_{B V^{k}} .
$$

This and Theorem $B$ further imply

$$
\|f\|_{C^{c, L_{1}}} \leq c(d)\left(\|f\|_{1}+|f|_{L i p_{1}^{d}}\right) .
$$


By definition the closed unit ball of $C^{c, L_{1}}$ is the closure of the closed unit ball of $C$ in $L_{1}$, hence, $\left(C^{c, L_{1}},\|\cdot\|_{C^{c, L_{1}}}\right)=\left(L_{\infty},\|\cdot\|_{\infty}\right)$. Using this and a homothety of $\mathbb{R}^{d}$ mapping $Q^{d}$ onto $Q$ we transform (9.23) to the inequality

$$
\|f\|_{L_{\infty}(Q)} \leq c(d)\left(|Q|^{-1}\|f\|_{L_{1}(Q)}+|f|_{L_{i p}(Q)}\right)
$$

Applying this to a function $f-m_{Q}$, where $m_{Q} \in \mathcal{P}_{k-1}^{d}$ is optimal for the distance from $f$ to $\mathcal{P}_{k-1}^{d}$ in $L_{1}(Q)$, we have

$$
E_{d \infty}(f ; Q) \leq\left\|f-m_{Q}\right\|_{L_{\infty}(Q)} \leq c(d)\left(|Q|^{-1} E_{d 1}(f ; Q)+|f|_{L_{i p}^{d}(Q)}\right) .
$$

Finally, by the Taylor formula for functions $g \in W_{1}^{d}(Q)$ we have

$$
E_{d 1}(g ; Q) \leq c(d)|Q| \cdot|g|_{W_{1}^{d}(Q)} .
$$

Since due to (9.22) the Banach space $\left(B V^{d}(Q),\|\cdot\|_{L_{1}(Q)}+|\cdot|_{B V^{d}(Q)}\right)$ coincides (up to equivalence of norms) with the relative completion of $W_{1}^{d}(Q)$ in $L_{1}(Q)$, for every $f \in$ $B V^{d}(Q)=\operatorname{Lip}_{1}^{d}(Q)$ (see Theorem A) there is a sequence $\left\{f_{n}\right\}_{n \in \mathbb{N}} \subset W_{1}^{d}(Q)$ such that $f_{n} \rightarrow f$ in $L_{1}(Q)$ and $\left|f_{n}\right|_{W_{1}^{d}(Q)} \rightarrow|f|_{B V^{d}(Q)}$ as $n \rightarrow \infty$.

Applying (9.25) to every $f_{n}$, then passing to limit and using Theorem A we obtain from (9.24) the required inequality

$$
E_{d \infty}(f ; Q) \leq c(d)\left(|Q|^{-1} \cdot|Q| \cdot|f|_{B V^{d}(Q)}+|f|_{L i p_{1}^{d}(Q)}\right) \leq c_{1}(d)|f|_{B V^{d}(Q)} .
$$

Lemma 9.4 is proved.

Now let $\pi$ be a packing in $Q^{d}$. Using Lemma 9.4 for each $Q \in \pi$ and $f \in B V^{d}$ we have

$$
\begin{aligned}
\sum_{Q \in \pi} E_{d \infty}(f ; Q) & \leq c(d) \sum_{Q \in \pi}|f|_{B V^{d}(Q)} \leq c(d) \max _{|\alpha|=d}\left\{\sum_{Q \in \pi} \operatorname{var}_{Q} D^{\alpha} f\right\} \\
& \leq c(d) \max _{|\alpha|=d} \operatorname{var}_{Q^{d}} D^{\alpha} f:=c(d)|f|_{B V^{d}} .
\end{aligned}
$$

Taking here supremum over $\pi$ we then obtain by Theorem A

$$
|f|_{V_{1 \infty}^{d}} \leq c(d)|f|_{B V^{d}} \leq c_{1}(d)|f|_{L_{i p}^{d}} .
$$

In other words, we proved the embedding

$$
\operatorname{Lip}_{1}^{d} \subset \dot{V}_{1 \infty}^{d} .
$$

This completes the proof of Proposition 9.3

To complete the proof of part (b) of the theorem, we consider the composition of continuous embeddings $i_{1}: N \dot{V}_{1}^{d} \hookrightarrow \operatorname{Lip}_{1}^{d}$, see (2.24), and $i_{2}: \operatorname{Lip}_{1}^{d} \subset \dot{V}_{1 \infty}^{d}$. The resulting embedding $i_{2} \circ i_{1}$ coincides with $\left.L\right|_{N \dot{V}_{1}^{d}}$, i.e., sends a function in $N \dot{V}_{1}^{d} \subset \ell_{\infty}$ to its equivalence class in $\dot{V}_{1 \infty}^{d} \subset L_{\infty}$. But according to Theorem 2.5(c) it maps $N \dot{V}_{1}^{d}$ isometrically onto $\dot{V}_{1 \infty}^{d}$. Hence, $\operatorname{Lip} p_{1}^{d}=\dot{V}_{1 \infty}^{d}$ and $N \dot{V}_{1}^{d} \cong \operatorname{Lip} p_{1}^{d}$.

The proof of Theorem 2.18 is complete. 
Proof of Corollary 2.20. First, we prove that each $\stackrel{\AA}{f} \in \dot{W}_{1}^{d}$ can be represented by a function $f \in A C$ (i.e., $f \in f$ ) such that

$$
|f|_{V_{1}^{d}} \leq c|\stackrel{\circ}{f}|_{W_{1}^{d}}
$$

for some $c$ depending only on $d$.

To this end we use the Gagliardo theorem [Ga-59] asserting that each $\stackrel{\circ}{g} \in \dot{W}_{1}^{d}(Q)$, $Q \subset Q^{d}$, can be represented by a (unique) continuous function $g$ and there is a linear projection $P_{Q}: L_{1}(Q) \rightarrow \mathcal{P}_{d-1}^{d}$ such that

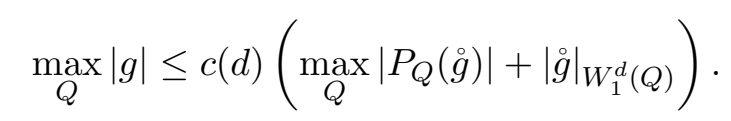

Let $f \in C$ be the representative of $\stackrel{\circ}{f}\left(\in \dot{W}_{1}^{d}\right)$. Applying (19.29) to $\stackrel{\circ}{g}=\left.\stackrel{\circ}{f}\right|_{Q}-P_{Q}\left(\left.\stackrel{\circ}{f}\right|_{Q}\right)$ and to its continuous representative $g:=\left.f\right|_{Q}-P_{Q}\left(\left.\stackrel{\circ}{f}\right|_{Q}\right)$ we obtain $\left(\right.$ as $\left.P_{Q}(g)=0\right)$ :

$$
E_{d}(f ; Q) \leq \max _{Q}\left|f-P_{Q}(\stackrel{\circ}{f})\right| \leq c(d)|\stackrel{\circ}{f}|_{W_{1}^{d}(Q)} .
$$

Then for every packing $\pi \in \Pi$

$$
\sum_{Q \in \pi} E_{d}(f ; Q) \leq c(d) \sum_{|\alpha|=d} \int_{\cup \pi}\left|D^{\alpha} f\right| d x,
$$

where $\cup \pi:=\cup_{Q \in \pi} Q$.

By the absolute continuity of the Lebesgue integral the right-hand side here tends to 0 as $|\cup \pi| \rightarrow 0$. Hence, $f$ satisfies condition (2.28), i.e., belongs to $A C$.

This and (9.30) prove the required statement (9.28).

Next, let $f \in A C\left(\subset \dot{V}_{1}^{d}\right)$. We prove that $L(A C) \subset \dot{W}_{1}^{d}$, where $L$ sends $f \in \dot{V}_{1}^{d}$ to its class of equivalence in $L_{\infty}$, and that

$$
|L(f)|_{W_{1}^{d}} \leq c|f|_{V_{1}^{d}}
$$

for some $c$ depending only on $d$.

This and (9.28) will show that $\left.L\right|_{A C}$ is an isomorphism of $A C$ onto $\dot{W}_{1}^{d}$.

We start with the embeddings $A C \subset\left(\dot{V}_{p}^{k}\right)^{0} \subset C$ implying that $A C \subset N \dot{V}_{1}^{d}$, see Section 5 and Corollary 2.2 (c). The latter, in turn, implies that $\left.L(f)\right|_{Q} \in B V^{d}(Q), Q \subset Q^{d}$, and, moreover,

$$
|f|_{V_{1}^{d}(Q)} \approx|L(f)|_{B V^{d}(Q)}
$$

with the constants of equivalence depending only on $d$, see (9.14), (9.17).

Hence, for every $\pi \in \Pi$

$$
\sum_{Q \in \pi}|L(f)|_{B V^{d}(Q)} \leq c(d) \sum_{Q \in \pi}|f|_{V_{1}^{d}(Q)}
$$

Choosing for every $Q \in \pi$ a packing $\pi_{Q} \in \Pi(Q)$ such that

$$
|f|_{V_{1}^{d}(Q)} \leq \sum_{R \in \pi_{Q}} E_{d}(f ; R)+\frac{\varepsilon}{\operatorname{card} \pi},
$$


where $\varepsilon>0$ is arbitrary small, we obtain from (9.32) that

$$
\sum_{|\alpha|=k} \operatorname{var}^{\operatorname{var}} D^{\alpha} L(f) \leq c(d)\left(\sum_{R \in \tilde{\pi}} E_{d}(f ; R)+\varepsilon\right),
$$

where $\tilde{\pi}:=\cup_{Q \in \pi} \pi_{Q}$ is a packing.

Sending $|\pi| \geq|\tilde{\pi}|$ to zero we conclude from here and (2.28) that for all closed sets $S$ being unions of nonoverlapping cubes, hence, for all Lebesgue measurable sets $S$

$$
\lim _{|S| \rightarrow 0} \operatorname{var}_{S} D^{\alpha} L(f)=0, \quad|\alpha|=d .
$$

By the Radon-Nikodym theorem, see, e.g., DSch-58, Sec. III.10],

$$
\left(D^{\alpha} L(f)\right)(S)=\int_{S} f_{\alpha} d x, \quad|\alpha|=d
$$

for all Lebesgue measurable sets $S \subset Q^{d}$ and some $f_{\alpha} \in L_{1}$.

In other words, $D^{\alpha} L(f) \in L_{1}$ for all $|\alpha|=d$ and $|L(f)|_{B V^{d}}=|L f|_{W_{1}^{d}}$.

Hence, $L$ maps $f \in A C$ in $\dot{W}_{1}^{d}$ and (9.31) is fulfilled.

This proves that $\left.L\right|_{A C}: A C \rightarrow \dot{W}_{1}^{d}$ is an isomorphism.

Finally, presentation (2.30) is valid for continuous representatives $f$ of elements $\stackrel{\circ}{f} \in \dot{W}_{1}^{d}$, see, e.g., Ma-85, Sec.1.10]. As we have proved such $f \in A C$, hence, this completes the proof of the corollary.

\section{Proof of Theorem 2.24}

10.1. Auxiliary Result. Let $\kappa: \dot{V}_{p \infty}^{k} \rightarrow V_{p \infty}^{k}:=\dot{V}_{p \infty}^{k} / \mathcal{P}_{k-1}^{d}$ be the quotient map defining the latter space. We then set

$$
\tilde{L}:=\kappa \circ\left(\left.L\right|_{\dot{V}_{p ; S}^{k}}\right)
$$

where $L: \dot{V}_{p}^{k} \rightarrow \dot{V}_{p \infty}^{k}$ is the surjective map of norm one sending a function in $\dot{V}_{p}^{k}$ to its equivalence class in $L_{\infty}$

By the definition, $\tilde{L}: \dot{V}_{p ; S}^{k} \rightarrow V_{p \infty}^{k}$ is a bounded surjective linear map of norm 1. (Recall that $\dot{V}_{p ; S}^{k} \subset \dot{V}_{p}^{k}$ is the subspace of functions vanishing on the interpolating set $S$ and that we naturally identify $\dot{V}_{p ; S}^{k}$ with $V_{p}^{k}$, see Section 4.1.1.)

In the next result, we use the duality between $V_{p ; S}^{k}\left(\equiv V_{p}^{k}\right)$ and $U_{p}^{k}$ established in Theorem 2.25 below and between $V_{p \infty}^{k}$ and $U_{p \infty}^{k}$ established in [BB-18, Th. 2.6].

Proposition 10.1. The map $\tilde{L}: \dot{V}_{p ; S}^{k} \rightarrow V_{p \infty}^{k}$ is weak ${ }^{*}$ continuous with respect to the weak* topologies on $\dot{V}_{p ; S}^{k}$ and $V_{p \infty}^{k}$.

Proof. It suffices to check that $\tilde{L}^{-1}(U) \subset \dot{V}_{p ; S}^{k}$ is weak* closed for each set $U$ of the form $\left\{f \in V_{p \infty}^{k}:|f(g)| \leq 1\right\}$, where $g \in U_{p \infty}^{k} \backslash\{0\}$. Since $\tilde{L}^{-1}(U)$ is a convex absorbing subset 
of $\dot{V}_{p ; S}^{k}$, it suffices by the Krein-Smulian theorem, see, e.g., [DSch-58, Thm. V.5.7], to check that $\tilde{L}^{-1}(U) \cap B\left(\dot{V}_{p ; S}^{k}\right)$ is closed in the weak* topology.

Let $\left\{f_{\alpha}\right\}_{\alpha \in \Lambda}$ be a net of functions in $\tilde{L}^{-1}(U) \cap B\left(\dot{V}_{p ; S}^{k}\right)$ converging in the weak* topology to a function $f \in B\left(\dot{V}_{p ; S}^{k}\right)$. According to Theorem 2.1, the space $\dot{V}_{p}^{k}$ consists of functions of the first Baire class (pointwise limits of continuous on $Q^{d}$ functions). Moreover, due to Lemma 4.1, the closed ball $B\left(\dot{V}_{p ; S}^{k}\right)$ is compact in the topology of pointwise convergence on $Q^{d}$. In particular, the net $\left\{f_{\alpha}\right\}_{\alpha \in \Lambda}$ pointwise converges to $f$. Since, in addition, $\sup _{\alpha}\left\|f_{\alpha}\right\|_{\infty} \leq 1$, the Rosenthal Main Theorem [Ro-77] implies that

$$
\lim _{\alpha} \int_{Q^{d}} f_{\alpha} d \mu=\int_{Q^{d}} f d \mu
$$

for all signed Borel measures $\mu$ on $Q^{d}$.

Now we apply (10.2) to $d \mu=h d x$ with $h \in \hat{L}_{1}:=\left\{g \in L_{1}: g \perp \mathcal{P}_{k-1}^{d}\right\}$. Since every function of $\hat{L}_{1}$ is an atom for the space $U_{p \infty}^{k}$, see the text before Theorem 2.24 in Section 2.3, $\hat{L}_{1}:=\left(U_{p \infty}^{k}\right)^{0} \subset U_{p \infty}^{k}$. Hence, we can use the duality pairing for $\left(V_{p \infty}^{k}, U_{p \infty}^{k}\right)$, see [BB-18, Th. 2.6], to get

$$
\lim _{\alpha}\left[\tilde{L}\left(f_{\alpha}\right)\right](h):=\lim _{\alpha} \int_{Q^{d}} f_{\alpha} h d x=\int_{Q^{d}} f h d x=:[\tilde{L}(f)](h) .
$$

Next, by the definition $U_{p \infty}^{k}$ is the completion of $\left(U_{p \infty}^{k}\right)^{0}$; hence, for every $g \in U_{p \infty}^{k}$ there is a sequence $\left\{g_{n}\right\}_{n \in \mathbb{N}} \subset \hat{L}_{1} \subset U_{p \infty}^{k}$ such that $\left\|g-g_{n}\right\|_{U_{p \infty}^{k}} \rightarrow 0$ as $n \rightarrow \infty$.

Applying (10.3) to $h=g_{n}$ we then obtain

$$
\lim _{\alpha}\left(\left[\tilde{L}\left(f_{\alpha}\right)\right]\left(g_{n}-g\right)+\left[\tilde{L}\left(f_{\alpha}\right)\right](g)-[\tilde{L}(f)](g)\right)=[\tilde{L}(f)]\left(g_{n}-g\right) .
$$

Further, since by our assumptions $\tilde{L}(f), \tilde{L}\left(f_{\alpha}\right) \in \tilde{L}\left(B\left(\dot{V}_{p ; S}^{k}\right)\right) \subseteq B\left(V_{p \infty}^{k}\right)$ for all $\alpha \in \Lambda$,

$$
\underset{\alpha}{\limsup }\left|\left[\tilde{L}\left(f_{\alpha}\right)\right]\left(g_{n}-g\right)\right|+\left|[\tilde{L}(f)]\left(g_{n}-g\right)\right| \leq 2\left\|g_{n}-g\right\|_{U_{p \infty}^{k}} \rightarrow 0 \quad \text { as } \quad n \rightarrow \infty .
$$

This and the previous imply that

$$
\underset{\alpha}{\limsup }\left|\left[\tilde{L}\left(f_{\alpha}\right)\right](g)-[\tilde{L}(f)](g)\right| \leq \lim _{n \rightarrow \infty}\left(\limsup _{\alpha}\left|\left[\tilde{L}\left(f_{\alpha}\right)\right]\left(g_{n}-g\right)\right|+\left|[\tilde{L}(f)]\left(g_{n}-g\right)\right|\right)=0,
$$

i.e., $\lim _{\alpha}\left[\tilde{L}\left(f_{\alpha}\right)\right](g)=[\tilde{L}(f)](g)$ for all $g \in U_{p \infty}^{k}$.

But $\tilde{L}\left(f_{\alpha}\right) \in U \cap B\left(V_{p \infty}^{k}\right)$ for all $\alpha \in \Lambda$; hence, $\tilde{L}(f)$ also belongs to $U \cap B\left(V_{p \infty}^{k}\right)$ since this set is closed in the weak* topology of $V_{p \infty}^{k}$. In particular,

$$
f \in \tilde{L}^{-1}(\tilde{L}(f)) \cap B\left(\dot{V}_{p ; S}^{k}\right) \subset \tilde{L}^{-1}(U) \cap B\left(\dot{V}_{p ; S}^{k}\right) .
$$

This shows that the set $\tilde{L}^{-1}(U) \subset \dot{V}_{p ; S}^{k}$ is weak* closed, as required.

The proof of the proposition is complete. 
10.2. Proof of Theorem 2.24. (a) We should prove that $U_{p}^{k}$ is a Banach space. Since $U_{p}^{k}$ is the completion of $\left(\left(U_{p}^{k}\right)^{0},\|\cdot\|_{\left(U_{p}^{k}\right)^{0}}\right)$, it suffices to prove that $\|\cdot\|_{\left(U_{p}^{k}\right)^{0}}$ is a norm, i.e., that if $\|g\|_{\left(U_{p}^{k}\right)^{0}}=0$ for some $g \in\left(U_{p}^{k}\right)^{0}$, then $g=0$.

To prove this, we first show that for every $f \in \dot{V}_{p}^{k}\left(\subset \ell_{\infty}\right)$ and $g \in\left(U_{p}^{k}\right)^{0}\left(\subset \ell_{1}\right)$

$$
\left|\sum_{x \in Q^{d}} f(x) g(x)\right| \leq|f|_{V_{p}^{k}}\|g\|_{U_{p}^{k}}
$$

Let $m_{Q} \in \mathcal{P}_{k-1}^{d}, Q \subset Q^{d}$, be such that

$$
\left\|f-m_{Q} ; Q\right\|_{\infty}=E_{k}(f ; Q)
$$

Then for $b_{\pi}:=\sum_{Q \in \pi} c_{Q} a_{Q}$ we obtain by the definition of $(k, p)$-atoms

$$
\sum_{x \in Q^{d}} f(x) b_{\pi}(x)=\sum_{Q \in \pi} c_{Q} \sum_{x \in Q}\left(f(x)-m_{Q}(x)\right) a_{Q}(x)
$$

Applying the Hölder inequality we derive from here

$$
\begin{aligned}
\left|\sum_{x \in Q^{d}} f(x) b_{\pi}(x)\right| & \leq\left(\sum_{Q \in \pi}\left|c_{Q}\right|^{p^{\prime}}\right)^{\frac{1}{p^{\prime}}}\left(\sum_{Q \in \pi}\left(\left\|f-m_{Q} ; Q\right\|_{\infty}\left\|a_{Q}\right\|_{1}\right)^{p}\right)^{\frac{1}{p}} \\
& \leq\left[b_{\pi}\right]_{p^{\prime}}\left(\sum_{Q \in \pi} E_{k}(f ; Q)^{p}\right)^{\frac{1}{p}} \leq\left[b_{\pi}\right]_{p^{\prime}}|f|_{V_{p}^{k}} .
\end{aligned}
$$

Since $g \in\left(U_{p}^{k}\right)^{0}$ can be presented as a finite sum of $(k, p)$-chains $b_{\pi}$, this gives

$$
\left|\sum_{x \in Q^{d}} f(x) g(x)\right| \leq\left(\inf \sum_{\pi}\left[b_{\pi}\right]_{p^{\prime}}\right)|f|_{V_{p}^{k}}=\|g\|_{U_{p}^{k}}|f|_{V_{p}^{k}} ;
$$

here infimum is taken over all such presentations of $g$.

From this inequality and the equality $\|g\|_{U_{p}^{k}}=0$ we deduce that

$$
\sum_{x \in Q^{d}} f(x) g(x)=0 \text { for all } f \in \dot{V}_{p}^{k} .
$$

In particular, since $C^{\infty} \subset \dot{\mathrm{v}}_{p}^{k} \subset \dot{V}_{p}^{k}$, see Section 5, equation (10.5) is valid for all $f \in C^{\infty}$.

Now assume, on the contrary, that $g$ satisfies (10.5) but $g \neq 0$. Let $X \subset Q^{d}$ be a finite subset of $\operatorname{supp} g$ such that

$$
\sum_{x \in X}|g(x)| \geq \frac{1}{2}\|g\|_{1}(>0)
$$

Clearly, there exists a function $\varphi_{X} \subset C^{\infty}$ such that

$$
\varphi_{X}(x)=\operatorname{sgn}(g(x)), \quad x \in X, \quad \text { and } \quad\left\|\varphi_{X}\right\|_{\infty} \leq 1 .
$$


From here and (10.5) we obtain the contradiction

$$
\frac{1}{2}\|g\|_{1} \leq \sum_{x \in X}|g(x)|=\left|\sum_{x \in X} \varphi_{X}(x) g(x)\right| \leq \sum_{x \in Q^{d} \backslash X}\left|\varphi_{X}(x)\left\|g(x) \mid<\frac{1}{2}\right\| g \|_{1} .\right.
$$

Hence, $g=0$ and the proof of part (a) of the theorem is complete.

(b) We should prove that $B\left(U_{p}^{k}\right)$ is the closure of the symmetric convex hull of the set $\left\{b_{\pi} \in\left(U_{p}^{k}\right)^{0}:\left[b_{\pi}\right]_{p^{\prime}} \leq 1\right\}$. This, in fact, is proved in Theorem 2.5 of [BB-18] for $B\left(U_{p \infty}^{k}\right)$. The proof can be easily adapted to the required case.

(c) We should prove that $U_{p}^{k}$ is nonseparable and contains a separable subspace. $\hat{U}_{p}^{k} \equiv U_{p \infty}^{k}$ such that $\left(U_{p}^{k} / \hat{U}_{p}^{k}\right)^{*} \cong \ell_{p}$.

Let us show that $U_{p}^{k}$ is nonseparable. To this end, we as in Lemma 4.1 take $S \subset Q^{d}$ to be an interpolating set for the space $\mathcal{P}_{k-1}^{d}$ and a function $c_{x}: S \rightarrow \mathbb{R}, x \in Q^{d}$, such that

$$
m(x)=\sum_{s \in S} c_{x}(s) m(s) \quad \text { for every } \quad m \in \mathcal{P}_{k-1}^{d} .
$$

Further, we set

$$
\delta_{x}^{\prime}:=\delta_{x}-\sum_{s \in S} c_{x}(s) \delta_{S}
$$

where $\delta_{y}$ is the delta-function at $y \in \mathbb{R}^{d}$.

If $x \neq y \in Q^{d} \backslash S$, then by definition

$$
\left\|\delta_{x}^{\prime}-\delta_{y}^{\prime}\right\|_{U_{p}^{k}}:=\sup _{f \in \dot{V}_{p ; S}^{k} ;|f|_{V_{p}^{k}} \leq 1}|f(x)-f(y)| \geq 2^{-\frac{1}{p}}\left|f_{x, y}(x)-f_{x, y}(y)\right|=2^{\frac{1}{p^{\prime}}} ;
$$

here $f_{x, y}(x)=1, f_{x, y}(y)=-1$ and $f_{x, y}(z)=0$ otherwise.

Hence, $U_{p}^{k}$ contains a discrete uncountable subset, i.e., it is nonseparable.

Next, let us show that $U_{p}^{k}$ contains a separable subspace $\hat{U}_{p}^{k}$ such that

$$
\hat{U}_{p}^{k} \equiv U_{p \infty}^{k} \quad \text { and } \quad\left(U_{p}^{k} / \hat{U}_{p}^{k}\right)^{*} \cong \ell_{p} .
$$

In fact, since $\tilde{L}: \dot{V}_{p ; S}^{k} \rightarrow V_{p \infty}^{k}$ is weak* continuous and surjective, see Proposition 10.1, there is a bounded linear injective map $T: U_{p \infty}^{k} \rightarrow U_{p}^{k}$ such that $T^{*}=\tilde{L}$, see, e.g., [DSch-58, VI.9.13].

We set

$$
\hat{U}_{p}^{k}:=T\left(U_{p \infty}^{k}\right)
$$

and prove that $T: U_{p \infty}^{k} \rightarrow\left(\hat{U}_{p}^{k},\|\cdot\|_{U_{p}^{k}}\right)$ is isometry.

Due to Theorem 2.5(c) $\tilde{L}$ maps the Banach space $N \dot{V}_{p}^{k} \cap \dot{V}_{p ; S}^{k}$ isometrically onto $V_{p \infty}^{k}$.

Further, let $u \in U_{p \infty}^{k} \backslash\{0\}$ and $f_{u} \in V_{p \infty}^{k}$ be such that $f_{u}(u)=\|u\|_{U_{p \infty}^{k}}$ and $\|f\|_{V_{p \infty}^{k}}=1$. Then there exists $\tilde{f}_{u} \in N \dot{V}_{p}^{k} \cap \dot{V}_{p ; S}^{k}$ such that $\tilde{L}\left(\tilde{f}_{u}\right)=f_{u}$ and $\left|\tilde{f}_{u}\right|_{V_{p}^{k}}=1$. In particular, we 
have

$$
\|T(u)\|_{U_{p}^{k}} \geq\left|\tilde{f}_{u}(T u)\right|=\left|\tilde{L}\left(\tilde{f}_{u}\right)(u)\right|=\left|f_{u}(u)\right|=\|u\|_{U_{p \infty}^{k}}
$$

On the other hand,

$$
\|T(u)\|_{U_{p}^{k}} \leq\|T\| \cdot\|u\|_{U_{p \infty}^{k}}=\|\tilde{L}\| \cdot\|u\|_{U_{p \infty}^{k}}=\|u\|_{U_{p \infty}^{k}}
$$

and therefore

$$
\|T(u)\|_{U_{p}^{k}}=\|u\|_{U_{p \infty}^{k}} \text { for all } u \in U_{p \infty}^{k}
$$

i.e., $T: U_{p \infty}^{k} \rightarrow\left(\hat{U}_{p}^{k},\|\cdot\|_{U_{p}^{k}}\right)$ is an isometric isomorphism and $\hat{U}_{p}^{k}$ is a closed subspace of $U_{p}^{k}$. It is separable because $U_{p \infty}^{k}$ is by $\mathrm{BB}-18$, Th. 2.5(b)].

Further, by the definition of dual to a factor-space, $\left(U_{p}^{k} / \hat{U}_{p}^{k}\right)^{*}$ is isomorphic to the annihilator $\left(\hat{U}_{p}^{k}\right)^{\perp} \subset V_{p}^{k}$ of $\hat{U}_{p}^{k}$. Moreover, since $T: U_{p \infty}^{k} \rightarrow \hat{U}_{p}^{k}$ is an isomorphism, an element $f \in\left(\hat{U}_{p}^{k}\right)^{\perp}$ iff for every $u \in U_{p \infty}^{k}$

$$
f(T(u))=(\tilde{L}(f))(u)=0,
$$

i.e., iff $f \in \operatorname{ker}(\tilde{L}) \cong \ell_{p}$.

This shows that $\left(U_{p}^{k} / \hat{U}_{p}^{k}\right)^{*} \cong \ell_{p}$ and completes the proof of the theorem.

\section{Proof of Theorem 2.25}

In the proof we use the following auxiliary result.

Lemma 11.1. Let $f \in \ell_{\infty}(Q)$. There is a sequence of functions $\left\{g_{n}\right\}_{\in \mathbb{N}} \in \ell_{1}(Q)$ such that

$$
\begin{gathered}
E_{k}(f ; Q)=\lim _{n \rightarrow \infty} \sum_{x \in Q} f(x) g_{n}(x) ; \\
\left\|g_{n}\right\|_{\ell_{1}(Q)}=1 \text { and } \sum_{x \in Q} x^{\alpha} g_{n}(x)=0, \quad|\alpha| \leq k-1, \quad \text { for all } n \in \mathbb{N} .
\end{gathered}
$$

Proof. Let $\hat{\ell}_{1}(Q):=\left\{g \in \ell_{1}(Q): g \perp \mathcal{P}_{k-1}^{d}\right\}$. By the Hahn-Banach theorem $\hat{\ell}_{1}(Q)^{*} \equiv$ $\ell_{\infty}(Q) / \mathcal{P}_{k-1}^{d}$. Since the best approximation in (11.1) is the norm of the image of $f$ in $\ell_{\infty}(Q) /\left.\mathcal{P}_{k-1}^{d}\right|_{Q}$, the definition of the norm of a bounded linear functional on $\hat{\ell}_{1}(Q)$ implies existence of a sequence $\left\{g_{n}\right\}_{n \in \mathbb{N}} \subset \hat{\ell}_{1}(Q)$ of elements of norm 1 satisfying (11.1)

Proof of Theorem 2.25. By definition, $\left(U_{p}^{k}\right)^{0} \subset \hat{\ell}_{1}$. Further, every function $f \in \hat{\ell}_{1}$ is a $(k, p)$-chain subordinate to the packing $\pi=\left\{Q^{d}\right\}$.

In fact, a function $f=c_{Q^{d}} a_{Q^{d}}$, where $c_{Q^{d}}:=\|f\|_{1}$ and $a_{Q^{d}}:=f /\|f\|_{1}$, vanishes on $\mathcal{P}_{k-1}^{d}$ and, moreover, $\left\|a_{Q^{d}}\right\|_{1}=1$. Hence, by the definition of the seminorm of $U_{p}^{k}$,

$$
\|f\|_{U_{p}^{k}} \leq\left|c_{Q^{d}}\right|=\|f\|_{1}
$$

In other words, the linear embedding

$$
E: \hat{\ell}_{1} \hookrightarrow U_{p}^{k}
$$


holds with the embedding constant 1 and has dense image. Passing to the conjugate map we obtain that

$$
E^{*}:\left(U_{p}^{k}\right)^{*} \hookrightarrow \hat{\ell}_{1}^{*} \equiv \ell_{\infty} / \mathcal{P}_{k-1}^{d}
$$

is a linear injection of norm $\leq 1$. On the other hand, $V_{p}^{k}$ is contained in $\ell_{\infty} / \mathcal{P}_{k-1}^{d}$.

Further, we show that range $\left(E^{*}\right)$ is in $V_{p}^{k}$ and that the linear map $E^{*}:\left(U_{p}^{k}\right)^{*} \rightarrow V_{p}^{k}$ is of norm $\leq 1$.

To this end, for $\ell \in\left(U_{p}^{k}\right)^{*}$ we denote by $f_{\ell} \in \ell_{\infty}$ an element whose image in $\ell_{\infty} / \mathcal{P}_{k-1}^{d}$ coincides with $E^{*}(\ell)$. Then we take for every $Q \subset Q^{d}$ and $\varepsilon \in\left(0, \frac{1}{2}\right)$ a $(k, p)$-atom denoted by $\tilde{a}_{Q}$ such that

$$
(1-\varepsilon) E_{k}\left(f_{\ell} ; Q\right) \leq \sum_{x \in Q} f_{\ell}(x) \tilde{a}_{Q}(x) \leq E_{k}\left(f_{\ell} ; Q\right) ;
$$

its existence directly follows from Lemma 11.1 and the definition of $(k, p)$-atoms.

Then for a $(k, p)$-chain $\tilde{b}_{\pi}$ given by $\tilde{b}_{\pi}:=\sum_{Q \in \pi} c_{Q} \tilde{a}_{Q}$ we get from (11.5)

$$
\left[E^{*}(\ell)\right]\left(\tilde{b}_{\pi}\right)=\sum_{x \in Q^{d}} \tilde{b}_{\pi}(x) f_{\ell}(x) \geq(1-\varepsilon) \sum_{Q \in \pi} c_{Q} E_{k}\left(f_{\ell} ; Q\right) .
$$

This, in turn, implies

$$
\begin{aligned}
\sum_{Q \in \pi} c_{Q} E_{k}\left(f_{\ell} ; Q\right) & \leq(1-\varepsilon)^{-1}\left\|E^{*}(\ell)\right\|_{1}\left\|\tilde{b}_{\pi}\right\|_{U_{p}^{k}} \leq(1-\varepsilon)^{-1}\|\ell\|_{\left(U_{p}^{k}\right)^{*}}\left\|\tilde{b}_{\pi}\right\|_{U_{p}^{k}} \\
& \leq(1-\varepsilon)^{-1}\left(\sum_{Q \in \pi}\left|c_{Q}\right|^{p^{\prime}}\right)^{\frac{1}{p^{\prime}}}\|\ell\|_{\left(U_{p}^{k}\right)^{*} \cdot}
\end{aligned}
$$

Sending here $\varepsilon \rightarrow 0$ and then taking supremum over all sequences $\left(c_{Q}\right)_{Q \in \pi}$ of the $\ell_{p^{\prime}}(\pi)$ norm 1 and supremum over all $\pi$ we conclude that

$$
\left|f_{\ell}\right|_{V_{p}^{k}}:=\sup _{\pi}\left(\sum_{Q \in \pi} E_{k}\left(f_{\ell} ; Q\right)^{p}\right)^{\frac{1}{p}} \leq\|\ell\|_{\left(U_{p}^{k}\right)^{*} \cdot}
$$

Hence, $E^{*}(\ell) \in V_{p}^{k}$ for every $\ell \in\left(U_{p}^{k}\right)^{*}$ and $E^{*}:\left(U_{p}^{k}\right)^{*} \hookrightarrow V_{p}^{k}$ is a linear injection of norm $\leq 1$.

Next, let us show that there is a linear injection $F$ of norm $\leq 1$

$$
F: V_{p}^{k} \hookrightarrow\left(U_{p}^{k}\right)^{*}
$$

such that

$$
F E^{*}=\left.\mathrm{id}\right|_{\left(U_{p}^{k}\right)^{*}}
$$

Actually, let $f \in \dot{V}_{p}^{k}$ and $\ell_{f}:\left(U_{p}^{k}\right)^{0} \rightarrow \mathbb{R}$ be a linear functional given for $g \in\left(U_{p}^{k}\right)^{0}$ by

$$
\ell_{f}(g):=\sum_{x \in Q^{d}} f(x) g(x) .
$$


Due to (10.4)

$$
\left|\ell_{f}(g)\right| \leq|f|_{V_{p}^{k}}\|g\|_{U_{p}^{k}}
$$

Thus, $\ell_{f}$ continuously extends to a linear functional from $\left(U_{p}^{k}\right)^{*}$ (denoted by the same symbol) and the linear map $F: V_{p}^{k} \rightarrow\left(U_{p}^{k}\right)^{*},\{f\}+\mathcal{P}_{k-1}^{d} \mapsto \ell_{f}$, is of norm $\leq 1$.

Further, we show that $F$ is an injection.

Indeed, let $\ell_{f}=0$ for some $f \in \dot{V}_{p}^{k}$. Since $\left(U_{p}^{k}\right)^{0}=\hat{\ell}_{1}$ and $\dot{V}_{p}^{k} \subset \ell_{\infty}$, equality (11.8) implies that $\left.\ell_{f}\right|_{\left(U_{p}^{k}\right)^{0}}$ determines the trivial functional on $\left(\hat{\ell}_{1}\right)^{*} \equiv \ell_{\infty} / \mathcal{P}_{k-1}^{d}$. Hence, $f \in$ $\mathcal{P}_{k-1}^{d}$, i.e., $f$ determines the zero element of the factor-space $V_{p}^{k}$, as required.

Further, by the definitions of $E^{*}$ and $F$ we have for each $h \in\left(U_{p}^{k}\right)^{*}$ and $g \in\left(U_{p}^{k}\right)^{0}$

$$
\left[F E^{*}(h)\right](g)=\ell_{E^{*}(h)}(g)=\sum_{x \in Q^{d}} E^{*}(h)(x) g(x)=h(E(g))=h(g) .
$$

The proof of (11.6) and (11.7) is complete.

In turn, the established results mean that $\operatorname{range}\left(E^{*}\right)=V_{p}^{k}, \operatorname{range}(F)=\left(U_{p}^{k}\right)^{*}$ and $F$ and $E^{*}$ are isometries.

The theorem is proved.

\section{Proof of Theorem 2.26}

(a) We should prove that the map sending a chain $b \in\left(U_{p}^{k}\right)^{0}\left(\subset \ell_{1}\right)$ to a discrete measure $\mu_{b} \in\left(\widetilde{U}_{p}^{k}\right)^{0}(\subset M)$, where $\mu_{b}(\{x\}):=b(x), x \in Q^{d}$, extends to an isometric embedding $\mathcal{I}$ of $U_{p}^{k}$ into $\widetilde{U}_{p}^{k}$.

In fact, by the definitions of seminorms of $\left(U_{p}^{k}\right)^{0}$ and $\left(\widetilde{U}_{p}^{k}\right)^{0}$, see Sections 2.3, 2.4, we obtain

$$
\left\|\mu_{b}\right\|_{\widetilde{U}_{p}^{k}} \leq\|b\|_{U_{p}^{k}} \quad \text { for all } \quad b \in\left(U_{p}^{k}\right)^{0}
$$

i.e., the map $\left(U_{p}^{k}\right)^{0} \ni b \mapsto \mu_{b} \in\left(\widetilde{U}_{p}^{k}\right)^{0}$ extends by continuity to a bounded linear map $\mathcal{I}: U_{p}^{k} \rightarrow \widetilde{U}_{p}^{k}$ of norm $\leq 1$.

The fact that $\mathcal{I}$ is an isometry is a consequence of the following properties of the introduced below operator $\mathcal{E}: \widetilde{U}_{p}^{k} \rightarrow U_{p}^{k}$ that will be established in part (b) of the proof:

$$
\mathcal{E} \circ \mathcal{I}=\text { id } \quad \text { and } \quad\|\mathcal{E}\| \leq 1 .
$$

Actually, these imply

$$
\|u\|_{U_{p}^{k}}=\|\mathcal{E}(\mathcal{I}(u))\|_{U_{p}^{k}} \leq\|\mathcal{I}(u)\|_{\widetilde{U}_{p}^{k}} \leq\|u\|_{U_{p}^{k}} \quad \text { for all } \quad u \in U_{p}^{k}
$$

Hence, $\mathcal{I}: U_{p}^{k} \rightarrow \widetilde{U}_{p}^{k}$ is an isometric embedding.

(b) We should prove that there exists a linear continuous surjection $\mathcal{E}: \widetilde{U}_{p}^{k} \rightarrow U_{p}^{k}$ such that

$$
\operatorname{ker}(\mathcal{E}) \cap\left(\widetilde{U}_{p}^{k}\right)^{0}=(0) \quad \text { and } \quad \mathcal{E} \circ \mathcal{I}=\mathrm{id} .
$$


To define such $\mathcal{E}$ we use a bilinear form given for $v \in \dot{V}_{p}^{k}$ and $\mu \in\left(\widetilde{U}_{p}^{k}\right)^{0}$ by

$$
\langle v, \mu\rangle:=\int_{Q^{d}} v d \mu
$$

Due to Theorem 2.1 this form is correctly defined. As we show now it can be continuously extended to the seminormed Banach space $\dot{V}_{p}^{k} \times \widetilde{U}_{p}^{k}$. (We will retain notation $\langle\cdot, \cdot\rangle$ for the extension.)

To this end it suffices to establish the inequality

$$
|\langle v, \mu\rangle| \leq|v|_{V_{p}^{k}}\|\mu\|_{\widetilde{U}_{p}^{k}}, \quad(v, \mu) \in \dot{V}_{p}^{k} \times\left(\widetilde{U}_{p}^{k}\right)^{0},
$$

whose proof repeats line by line the proof of inequality (10.4) with sum replaced by integral, $a_{Q} \in \ell_{1}$ by $\mu_{Q} \in M$ and $\|\cdot\|$ by $\|\cdot\|_{M}$. We leave the details to the readers.

Thus, inequality (12.3) is valid for all $\mu \in \widetilde{U}_{p}^{k}$ and, hence, for every such $\mu$ the map

$$
\ell_{\mu}: v \mapsto\langle v, \mu\rangle, \quad v \in \dot{V}_{p ; S}^{k}\left(\equiv V_{p}^{k}\right),
$$

determines a linear continuous functional from $\left(V_{p}^{k}\right)^{*}$.

Now we define the required map $\mathcal{E}$ by the formula

$$
\mathcal{E}(\mu):=\ell_{\mu}, \quad \mu \in \widetilde{U}_{p}^{k} .
$$

Then $\mathcal{E}$ acts linearly from $\widetilde{U}_{p}^{k}$ to $\left(V_{p}^{k}\right)^{*}$ and is of norm $\leq 1$. We should prove that the range of $\mathcal{E}$ coincides with $U_{p}^{k}\left(\subset\left(U_{p}^{k}\right)^{* *} \equiv\left(V_{p}^{k}\right)^{*}\right)$.

To this end we establish the following:

Each bounded linear functional $\ell_{\mu}$ with $\mu \in\left(\widetilde{U}_{p}^{k}\right)^{0}$ is weak* continuous on $V_{p}^{k}$ with respect to the weak $k^{*}$ topology induced by the duality $\left(U_{p}^{k}\right)^{*} \equiv V_{p}^{k}$.

It suffices to check that $\ell_{\mu}^{-1}([-1,1]) \subset V_{p}^{k}, \ell_{\mu}:=\mathcal{E}(\mu)$, is weak* closed. In fact, since $\ell_{\mu}^{-1}([-1,1])$ is a convex absorbing subset of $V_{p}^{k}$, it suffices by the Krein-Smulian theorem, see, e.g., DSch-58, Thm. V.5.7], to check that $\ell_{\mu}^{-1}([-1,1]) \cap B\left(V_{p}^{k}\right)$ is closed in the weak* topology. In the proof of this, without loss of generality we identify $V_{p}^{k}$ with $\dot{V}_{p ; S}^{k} \subset \dot{V}_{p}^{k}$, see Section 4.1.1, so that $B\left(V_{p}^{k}\right)=B\left(\dot{V}_{p ; S}^{k}\right)$.

Let $\left\{f_{\alpha}\right\}_{\alpha \in \Lambda}$ be a net of functions in $\ell_{\mu}^{-1}([-1,1]) \cap B\left(\dot{V}_{p ; S}^{k}\right)$ converging in the weak* topology to a function $f \in B\left(\dot{V}_{p ; S}^{k}\right)$. Since $\dot{V}_{p}^{k}$ consists of functions of the first Baire class, see Theorem 2.1, and the closed ball $B\left(\dot{V}_{p ; S}^{k}\right)$ is compact in the topology of pointwise convergence on $Q^{d}$ (see Lemma 4.1), the net $\left\{f_{\alpha}\right\}_{\alpha \in \Lambda}$ pointwise converges to $f$; in addition, $\sup _{\alpha}\left\|f_{\alpha}\right\|_{\infty} \leq 1$ and $\left\|\ell_{\mu}\right\| \leq 1$. These and the Rosenthal Main Theorem [Ro-77] imply that

$$
\ell_{\mu}(f):=\int_{Q^{d}} f d \mu=\int_{Q^{d}} \lim _{\alpha} f_{\alpha} d \mu=\lim _{\alpha} \int_{Q^{d}} f_{\alpha} d \mu=\lim _{\alpha} \ell_{\mu}\left(f_{\alpha}\right)(\in[-1,1]) ;
$$

hence, $f \in \ell_{\mu}^{-1}([-1,1])$ and $\ell_{\mu} \in\left(V_{p}^{k}\right)^{*}$ is weak $^{*}$ continuous. 
Since the subspace of weak* continuous functionals in $\left(V_{p}^{k}\right)^{*}$ coincides with $U_{p}^{k}$, we conclude that $\mathcal{E}(\mu) \in U_{p}^{k}$ for all $\mu \in\left(\widetilde{U}_{p}^{k}\right)^{0}$. Moreover, $U_{p}^{k}$ is a closed subspace of $\left(V_{p}^{k}\right)^{*}$ and $\mathcal{E}$ is continuous on $\left(\widetilde{U}_{p}^{k}\right)^{0}$ that is dense in $\widetilde{U}_{p}^{k}$. These facts imply that $\operatorname{range}(\mathcal{E}) \subset U_{p}^{k}$.

Next, we show that

$$
\operatorname{ker}(\mathcal{E}) \cap\left(\widetilde{U}_{p}^{k}\right)^{0}=(0) .
$$

In fact, let $\mu \in \operatorname{ker}(\mathcal{E}) \cap\left(\widetilde{U}_{p}^{k}\right)^{0}$. Then for each $f \in C^{\infty} \subset \dot{V}_{p}^{k}$

$$
(\mathcal{E}(\mu))(f):=\int_{Q^{d}} f d \mu=0 .
$$

Since $C^{\infty}$ is dense in $C$ in the topology of uniform convergence, the latter implies that

$$
\int_{Q^{d}} f d \mu=0 \text { for all } f \in C .
$$

According to the Riesz representation theorem $C^{*} \equiv\left(M,\|\cdot\|_{M}\right)$ with the duality defined by integration with respect to the corresponding measure; hence, (12.7) implies that $\mu=0$ proving (12.6).

Finally, we prove that

$$
\mathcal{E} \circ \mathcal{I}=\text { id }
$$

In fact, we have for $b \in\left(U_{p}^{k}\right)^{0}\left(\subset \ell_{1}\right)$ and all $v \in \dot{V}_{p ; S}^{k}\left(\subset \ell_{\infty}\right)$

$$
(\mathcal{E}(\mathcal{I}(b)))(v)=\int_{Q} v d \mu_{b}=\sum_{x \in Q^{d}} v(x) b(x)=b(v) .
$$

Since by Theorem $2.25\left(U_{p}^{k}\right)^{*} \equiv V_{p}^{k}$, the latter implies that

$$
\mathcal{E}(\mathcal{I}(b))=b \quad \text { for all } \quad b \in\left(U_{p}^{k}\right)^{0} .
$$

From here using that $\mathcal{E}$ and $\mathcal{I}$ are continuous maps and that $\left(U_{p}^{k}\right)^{0}$ is dense in $U_{p}^{k}$ we obtain the required identity (12.8).

In particular, this implies that range $(\mathcal{E})=U_{p}^{k}$, i.e., surjectivity of $\mathcal{E}$, and completes the proof of part (b) and hence of the theorem.

\section{Proofs of Theorem 2.27 And Corollary 2.28}

Proof of Theorem 2.27. We have to prove that if

$$
s:=\frac{d}{p}<k, \quad 1<p<\infty
$$

then

$$
\left(\mathrm{v}_{p}^{k}\right)^{*} \cong U_{p}^{k}
$$

Since the relation

$$
\left(\mathrm{v}_{p \infty}^{k}\right)^{*} \cong U_{p \infty}^{k}
$$


under condition (13.1) has been just proved in [BB-18, Th. 2.7] and the spaces of our consideration are defined similarly to those in (13.3), several of the basic arguments of the cited paper can and will after some trivial modification of notations be used in the forthcoming proof.

In the following text we as before identify $U_{p}^{k}$ with its image under the natural embedding $U_{p}^{k} \hookrightarrow\left(U_{p}^{k}\right)^{* *}$. and $V_{p}^{k}$ with $\left(U_{p}^{k}\right)^{*}$, see Theorem 2.25, hence, we regard $U_{p}^{k}$ as a linear subspace of $\left(V_{p}^{k}\right)^{*}\left(=\left(\left(U_{p}^{k}\right)^{*}\right)^{*}\right)$.

Further, $\mathfrak{i}: \mathrm{V}_{p}^{k} \hookrightarrow V_{p}^{k}$ is the natural embedding and $\mathfrak{i}^{*}:\left(V_{p}^{k}\right)^{*} \rightarrow\left(\mathrm{v}_{p}^{k}\right)^{*}$ is its adjoint.

Proposition 13.1. (a) $\mathfrak{i}^{*}$ is a surjective linear map of norm one which maps $U_{p}^{k}$ isomorphically onto a closed subspace of $\left(\mathrm{V}_{p}^{k}\right)^{*}$.

(b) The image $\mathrm{i}^{*}\left(B\left(U_{p}^{k}\right)\right)$ is a dense subset of $B\left(\left(\mathrm{v}_{p}^{k}\right)^{*}\right)$ in the weak topology of $\left(\mathrm{v}_{p}^{k}\right)^{*}$.

Proof. The proof that $\mathrm{i}^{*}:\left(V_{p}^{k}\right)^{*} \rightarrow\left(\mathrm{v}_{p}^{k}\right)^{*}$ is a surjective linear map of norm one repeats line by line the proof of the similar assertion of Proposition 6.1 in [BB-18]. The same is true for part (b) of Proposition 13.1.

To prove the remaining statement of part (a) of the proposition we use the following analog of Lemma 6.2 of [BB-18].

Lemma 13.2. The subspace $\mathrm{V}_{p}^{k}$ is weak* dense in the space $V_{p}^{k}\left(=\left(U_{p}^{k}\right)^{*}\right)$.

Proof. The result is a straightforward corollary of Theorem 2.3.

Now, to prove that $\mathfrak{i}^{*}$ maps $U_{p}^{k}$ isomorphically onto a closed subspace of $\left(\mathrm{v}_{p}^{k}\right)^{*}$, we have to show that there is a constant $c \in(0,1)$ such that $\left\|\mathfrak{i}^{*}(v)\right\|_{\left(\mathrm{v}_{p}^{k}\right)^{*}} \geq c\|v\|_{U_{p}^{k}}$ for all $v \in U_{p}^{k}$.

In fact, let $u \in U_{p}^{k} \backslash\{0\}$. By the Hahn-Banach theorem there exists $f \in V_{p}^{k}$ such that $\|f\|_{V_{p}^{k}}=1$ and $f(u)=\|u\|_{U_{p}^{k}}$. By Theorem 2.3 there exists a sequence $\left\{f_{n}\right\}_{n \in \mathbb{N}} \subset \mathrm{v}_{p}^{k}$ weak $^{*}$ converging to $f$ such that

$$
\sup _{n}\left\|f_{n}\right\|_{V_{p}^{k}} \leq C\|f\|_{V_{p}^{k}}=C
$$

for some $C \geq 1$ independent of $f$.

These imply that

$$
\|v\|_{U_{p}^{k}}=|f(v)|=\lim _{n \rightarrow \infty}\left|f_{n}(v)\right| \leq C \sup _{g \in B\left(\mathrm{v}_{p}^{k}\right)}|g(v)|=C \sup _{g \in B\left(\mathrm{v}_{p}^{k}\right)}\left|\left(\mathfrak{i}^{*}(v)\right)(g)\right|:=C\left\|\mathfrak{i}^{*}(v)\right\|_{\left(\mathrm{v}_{p}^{k}\right)^{*}}
$$

as required.

This completes the proof of Proposition 13.1 .

Our next result is the analog of Proposition 6.3 of BB-18. In its formulation, we use Theorem 2.26 to identify the vector space of measures $\hat{M}:=\left(\widetilde{U}_{p}^{k}\right)^{0}$ vanishing on $\mathcal{P}_{k-1}^{d}$ with a subspace of $U_{p}^{k}$. Let $\overline{\mathscr{B}}_{p}^{k}$ stand for the closure in $U_{p}^{k}$ of the set of $(k, p)$-chains

$$
\mathscr{B}_{p}^{k}:=\left\{b_{\pi} \in\left(\widetilde{U}_{p}^{k}\right)^{0}:\left[b_{\pi}\right]_{p^{\prime}} \leq 1\right\} \subset \hat{M},
$$

see (2.31) for the definition. 
Proposition 13.3. $\mathfrak{i}^{*}\left(\overline{\mathscr{B}}_{p}^{k}\right)$ is a subset of $B\left(\left(\mathrm{v}_{p}^{k}\right)^{*}\right)$ compact in the weak $k^{*}$ topology of $\left(\mathrm{v}_{p}^{k}\right)^{*}$. Proof. As in the proof of [BB-18, Prop. 6.3], the required result follows from the next one.

Statement 13.4. If $\left\{b^{n}\right\}_{i \in \mathbb{N}} \subset \mathscr{B}_{p}^{k}$ is such that the sequence $\left\{\mathfrak{i}^{*}\left(b^{n}\right)\right\}_{n \in \mathbb{N}}$ weak $k^{*}$ converges in $B\left(\left(\mathrm{v}_{p}^{k}\right)^{*}\right)$, then its limit belongs to $\mathrm{i}^{*}\left(\overline{\mathscr{B}}_{p}^{k}\right)$.

Proof. Let $b^{n}$ has the form

$$
b^{n}:=\sum_{i=1}^{N(n)} c_{i}^{n} \mu_{Q_{i}^{n}}, \quad n \in \mathbb{N},
$$

where $\pi_{n}:=\left\{Q_{i}^{n}: 1 \leq i \leq N(n)\right\} \in \Pi$ is a packing.

As in the cited proposition we assume without loss of generality that

$$
\left|Q_{i+1}^{n}\right| \leq\left|Q_{i}^{n}\right|, \quad 1 \leq i<N(n) .
$$

Further, we extend sequences $\pi_{n},\left\{c_{i}^{n}\right\}$ and $\left\{\mu_{Q_{i}^{n}}\right\}$ by setting

$$
Q_{i}^{n}:=\{0\}, \quad c_{i}^{n}:=0, \quad \mu_{Q_{i}^{n}}:=0 \quad \text { for } \quad i>N(n) .
$$

Hence, we write

$$
b^{n}:=\sum_{i=1}^{\infty} c_{i}^{n} \mu_{Q_{i}^{n}}, \quad n \in \mathbb{N} .
$$

Lemma 13.5. There is an infinite subsequence $\left\{b^{n}\right\}_{n \in J}, J \subset \mathbb{N}$, such that for every $i \in \mathbb{N}$ the following is true.

(a) $\left\{Q_{i}^{n}\right\}_{n \in J}$ converges in the Hausdorff metric to a closed subcube of $Q^{d}$ denoted by $Q_{i}$

(b) $\left\{\mathrm{i}^{*}\left(\mu_{Q_{i}^{n}}\right)\right\}_{n \in \mathbb{N}} \subset B\left(\left(\mathrm{v}_{p}^{k}\right)^{*}\right)$ converges in the weak $k^{*}$ topology of $B\left(\left(\mathrm{v}_{p}^{k}\right)^{*}\right)$;

(c) if the limiting cube $Q_{i}$ has a nonempty interior, then the sequence of measures $\left\{\mu_{Q_{i}^{n}}\right\}_{n \in J}$ converges in the weak ${ }^{*}$ topology of $M$ (regarded as the dual space of $\left.C:=C\left(Q^{d}\right)\right)$;

(d) the sequence $\left\{c^{n}:=\left(c_{i}^{n}\right)_{i \in \mathbb{N}}\right\}_{n \in J}$ of vectors from $B\left(\ell_{p^{\prime}}(\mathbb{N})\right)$ converges in the weak $k^{*}$ topology of $\ell_{p^{\prime}}(\mathbb{N})\left(=\ell_{p}(\mathbb{N})^{*}\right)$ to a vector denoted by $c\left(\in B\left(\ell_{p^{\prime}}(\mathbb{N})\right)\right)$.

Proof. The result, in fact, is proved in [BB-18, Lm. 6.5].

Hence, without loss of generality we can and will assume that the sequence $\left\{b^{n}\right\} \subset \mathscr{B}_{p}^{k}$ of $(k, p)$-chains satisfies the assertions of Lemma 13.5. In particular, there are closed cubes $Q_{i} \subset Q^{d}, i \in \mathbb{N}$, such that in the Hausdorff metric

$$
Q_{i}=\lim _{n \rightarrow \infty} Q_{i}^{n}
$$

Since for each $n \in \mathbb{N}$ the cubes $Q_{i}^{n}, i \in \mathbb{N}$, are nonoverlapping and their volumes form a nonincreasing sequence, the same is true for the family of cubes $\left\{Q_{i}\right\}_{i \in \mathbb{N}}$. Thus, for every $i \in \mathbb{N}$

$$
\stackrel{\circ}{Q}_{i} \cap \stackrel{\circ}{Q}_{i+1}=\emptyset \text { and }\left|Q_{i}\right| \geq\left|Q_{i+1}\right|
$$


Now we let $N=\infty$ if $\left|Q_{i}\right| \neq 0$ for all $i \in \mathbb{N}$, otherwise, $N$ be the minimal element of the set of integers $n \in \mathbb{Z}_{+}$such that

$$
\left|Q_{i}\right|=0 \text { for } i>n \text {. }
$$

Then due to Lemma 13.5(c) for $N \neq 0$ there are measures $\mu_{i} \in M, 1 \leq i<N+1$, such that in the weak* topology of $M$

$$
\mu_{i}=\lim _{n \rightarrow \infty} \mu_{Q_{i}^{n}}
$$

Lemma 13.6. (1) If $N \neq \infty$ and $i>N$, then in the weak $k^{*}$ topology of $B\left(\left(\mathrm{v}_{p}^{k}\right)^{*}\right)$

$$
\lim _{n \rightarrow \infty} i^{*}\left(\mu_{Q_{i}^{n}}\right)=0 .
$$

(2) If $N \neq 0$ and $1 \leq i<N+1$, then the measure $\mu_{i}$ is a $(k, p)$-atom subordinate to $Q_{i}$.

Proof. The proof of part (1) repeats with the corresponding change of notations and definitions the analogous assertion in Lemma 6.6 of [BB-18].

(2) First, due to (13.8)

$$
\left\|\mu_{i}\right\|_{M} \leq 1
$$

as atoms $\mu_{Q_{i}^{n}}$ belong to the closed unit ball of $M=C^{*}$ which is weak* compact.

Next, we prove that

$$
\operatorname{supp} \mu_{i} \subset Q_{i} \quad \text { and } \quad \mu_{i} \perp \mathcal{P}_{k-1}^{d} .
$$

If, on the contrary, supp $\mu_{i} \backslash Q_{i} \neq \emptyset$, then there is a continuous function $f \in C$ such that $(\operatorname{supp} f) \cap Q_{i}=\emptyset$ and

$$
\int_{Q^{d}} f d \mu_{i} \neq 0
$$

However, $Q_{i}^{n} \rightarrow Q_{i}$ in the Hausdorff metric as $n \rightarrow \infty$ and therefore $Q_{i}^{n} \cap \operatorname{supp} f=\emptyset$ for all sufficiently large $n$. This and condition (c) of Lemma 13.5 imply that

$$
0=\lim _{n \rightarrow \infty} \int_{Q^{d}} f d \mu_{Q_{i}^{n}}=\int_{Q} f d \mu_{i}
$$

in contradiction with (13.11).

To prove the second assertion of (13.10) we note that $\mu_{Q_{i}^{n}} \perp \mathcal{P}_{k-1}^{d}$ for all $i$ and $n$. Then according to condition (c) of Lemma 13.5 we have for each $m \in \mathcal{P}_{k-1}^{d}$

$$
\int_{Q^{d}} m d \mu_{i}=\int_{Q^{d}} m d \mu_{Q_{i}^{n}}=0
$$

as required.

Hence, $\mu_{i}$ (in the sequel denoted by $\mu_{Q_{i}}$ ) is a $(k, p)$-atom subordinate to $Q_{i}$.

The proof of the lemma is complete. 
Now we show that for $1 \leq N<\infty$

$$
v_{N}:=\sum_{i=1}^{N} c_{i} \mu_{Q_{i}} \in \mathscr{B}_{p}^{k}
$$

In fact, by Lemma $13.6(2)$ and (13.6) $\mu_{Q_{i}}$ are $(k, p)$-atoms and $\left\{Q_{i}\right\}_{1 \leq i \leq N}$ is a packing. Moreover, by Lemma 13.5(d) the $(k, p)$-atom $v_{N}$ satisfies

$$
\left[v_{N}\right]_{p^{\prime}}=\|c\|_{p^{\prime}} \leq 1
$$

as required.

Further, for $N=\infty$

$$
v_{\infty}:=\sum_{i=1}^{\infty} c_{i} \mu_{Q_{i}} \in \overline{\mathscr{B}}_{p}^{k}
$$

In fact, by Lemmas 13.5(d) and 13.6(2), and inequality (12.3),

$$
\left\|\sum_{i=\ell}^{m} c_{i} \mu_{Q_{i}}\right\|_{U_{p}^{k}} \leq\left(\sum_{i=\ell}^{m}\left|c_{i}\right|^{p^{\prime}}\right)^{\frac{1}{p^{\prime}}} \rightarrow 0
$$

as $\ell, m \rightarrow \infty$, i.e., the series in (13.13) converges in $U_{p}^{k}$. Moreover, its partial sums belong to $\mathscr{B}_{p}^{k}$, cf. (13.12), hence, $v_{\infty}$ belongs to the closure of $\mathscr{B}_{p}^{k}$.

In the remaining case of $N=0$ we set

$$
v_{0}:=0 .
$$

Now the proof of Statement 13.4 will be completed if we show that

$$
\lim _{n \rightarrow \infty} \mathfrak{i}^{*}\left(b^{n}\right)=\mathfrak{i}^{*}\left(v_{N}\right)
$$

in the weak* topology of $B\left(\left(\mathrm{v}_{p}^{k}\right)^{*}\right)$.

However, the derivation of (13.15) repeats line by line the proof of the similar statement in BB-18, see equation (6.23) there.

The proof of Statement 13.4 is complete.

Now, we complete the proof of Theorem 2.27 beginning with the following:

Lemma 13.7. The space $\mathrm{v}_{p}^{k}$ isometrically embeds in the space $C\left(\mathfrak{i}^{*}\left(\overline{\mathscr{B}}_{p}^{k}\right)\right)$ of continuous functions on the (metrizable) compact space $\mathfrak{i}^{*}\left(\overline{\mathscr{B}}_{p}^{k}\right)$,

Proof. By Theorem 2.24(b) $B\left(U_{p}^{k}\right)$ is the closure of the symmetric convex hull of the set $\left\{b_{\pi} \in\left(U_{p}^{k}\right)^{0}:\left[b_{\pi}\right]_{p^{\prime}} \leq 1\right\}$. Since the latter set is a subset of $\mathscr{B}_{p}^{k} \subset B\left(U_{p}^{k}\right)$, see (13.4), $B\left(U_{p}^{k}\right)$ is the closure of the symmetric convex hull of $\mathscr{B}_{p}^{k}$, denoted by $\operatorname{sc}\left(\mathscr{B}_{p}^{k}\right)$. As in [BB-18, Lm.6.7] this implies that the set

$$
\mathfrak{i}^{*}\left(\operatorname{sc}\left(\overline{\mathscr{B}}_{p}^{k}\right)\right)=\operatorname{sc}\left(\mathfrak{i}^{*}\left(\overline{\mathscr{B}}_{p}^{k}\right)\right)
$$


is weak ${ }^{*}$ dense in $B\left(\left(\mathrm{v}_{p}^{k}\right)^{*}\right)$ and for every element $v \in \mathrm{v}_{p}^{k}$ regarded as a bounded linear functional on $\left(\mathrm{v}_{p}^{k}\right)^{*}$

$$
\|v\|_{\mathrm{V}_{p}^{k}}=\sup _{v^{*} \in \mathfrak{i}^{*}\left(\overline{\mathscr{B}}_{p}^{k}\right)}\left|v\left(v^{*}\right)\right| .
$$

Since $\left.v\right|_{\mathfrak{i}^{*}\left(\overline{\mathscr{B}}_{p}^{k}\right)}$ is a continuous function on $\mathfrak{i}^{*}\left(\overline{\mathscr{B}}_{p}^{k}\right)$ in the weak ${ }^{*}$ topology induced from $B\left(\left(\mathrm{~V}_{p}^{k}\right)^{*}\right)$ and its supremum norm equals $\|v\|_{\mathrm{v}_{p}^{k}}$, the map

$$
\mathrm{V}_{p}^{k} \ni v \mapsto v\left(v^{*}\right), \quad v^{*} \in \mathfrak{i}^{*}\left(\overline{\mathscr{B}}_{p}^{k}\right),
$$

is a linear isometric embedding of $\mathrm{v}_{p}^{k}$ in $C\left(\mathfrak{i}^{*}\left(\overline{\mathscr{B}}_{p}^{k}\right)\right)$.

Now let $v^{*}$ be a linear continuous functional on the space $\mathrm{V}_{p}^{k}$ regarded as the closed subspace of the space $C\left(\mathfrak{i}^{*}\left(\overline{\mathscr{B}}_{p}^{k}\right)\right)$. By the Hahn-Banach theorem $v^{*}$ can be extended to a linear continuous functional, say, $\hat{v}^{*}$ on this space with the same norm. In turn, by the Riesz representation theorem there is a regular finite (signed) Borel measure on the compact space $\mathfrak{i}^{*}\left(\overline{\mathscr{B}}_{p}^{k}\right)$ denoted by $\mu_{v^{*}}$ that represents $\hat{v}^{*}$.

This implies that

$$
v\left(v^{*}\right)=\int_{i^{*}\left(\overline{\mathscr{B}}_{p}^{k}\right)} v d \mu_{v^{*}}, \quad v \in \mathrm{v}_{p}^{k}
$$

Now we use this measure to find a similar representation for elements of $V_{p}^{k}$.

Since $\mathrm{V}_{p}^{k}$ is a weak ${ }^{*}$ dense subspace of the space $V_{p}^{k}$, see Lemma 13.2 and Theorem 2.3 , for every $v \in V_{p}^{k}$ there is a bounded in the $\mathrm{V}_{p}^{k}$ norm sequence $\left\{v_{j}\right\}_{j \in \mathbb{N}} \subset \mathrm{V}_{p}^{k}$ such that

$$
\lim _{j \rightarrow \infty} v_{j}(u)=v(u), \quad u \in U_{p}^{k} .
$$

Now let $\tau: \mathfrak{i}^{*}\left(U_{p}^{k}\right) \rightarrow U_{p}^{k}$ be the inverse to the injection $\left.\mathfrak{i}^{*}\right|_{U_{p}^{k}}: U_{p}^{k} \rightarrow\left(\mathrm{v}_{p}^{k}\right)^{*}$, see Proposition 13.1(a). Making the change of variable $u \rightarrow \tau\left(v^{*}\right)$ we derive from (13.19)

$$
\lim _{j \rightarrow \infty} v_{j}\left(v^{*}\right)=(v \circ \tau)\left(v^{*}\right), \quad v^{*} \in \mathfrak{i}^{*}\left(\overline{\mathscr{B}}_{p}^{k}\right) .
$$

Since linear functionals $v_{j}: \mathrm{v}_{p}^{k} \rightarrow \mathbb{R}$ are continuous in the weak* topology defined by $\left(\mathrm{v}_{p}^{k}\right)^{*}$ their traces to $\mathfrak{i}^{*}\left(\overline{\mathscr{B}}_{p}^{k}\right)$ are continuous functions in the weak ${ }^{*}$ topology induced from $B\left(\left(\mathrm{~V}_{p}^{k}\right)^{*}\right)$. This implies the following:

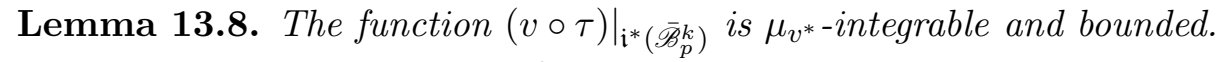

Moreover, a function $\phi_{v^{*}}: V_{p}^{k} \rightarrow \mathbb{R}$ given by

$$
\phi_{v^{*}}(v):=\int_{\mathfrak{i}^{*}\left(\overline{\mathscr{B}}_{p}^{k}\right)} v \circ \tau d \mu_{v^{*}}
$$

belongs to $\left(V_{p}^{k}\right)^{*}$.

Proof. The result is, in fact, proved in Lemma 6.8 of [BB-18]. 
At the next stage we establish weak* continuity of $\phi_{v^{*}}$ on $V_{p}^{k}$ regarded as the dual space of $U_{p}^{k}$, see Theorem 2.25.

To this end it suffices to show that $\phi_{v^{*}}^{-1}(R) \subset V_{p}^{k}$ is weak* closed for every closed interval $R \subset \mathbb{R}$. Since this preimage is convex, we can use the Krein-Smulian weak ${ }^{*}$ closedness criterion, see, e.g., DSch-58, Thm. V.5.7]. In our case, it asserts that $\phi_{v^{*}}^{-1}(R)$ is weak* closed iff $B_{r}(0) \cap \phi_{v^{*}}^{-1}(R)$ is for every $r>0$; here $B_{r}(0):=\left\{v \in V_{p}^{k}:\|v\|_{V_{p}^{k}} \leq r\right\}$.

Without loss of generality we identify $\left(V_{p}^{k},\|\cdot\|_{V_{p}^{k}}\right)$ with $\left(\dot{V}_{p ; S}^{k},|\cdot|_{V_{p}^{k}}\right)$, where $\dot{V}_{p ; S}^{k} \subset$ $\dot{V}_{p}^{k}$ is the subspace of functions vanishing on an interpolating set $S \subset Q^{d}$ for $\mathcal{P}_{k-1}^{d}$, see Section 4.1.1.

Further, let $\mathcal{B}_{1}\left(Q^{d}\right)$ stand for the space of functions on $Q^{d}$ of the first Baire class equipped with the topology of pointwise convergence. By Lemma 4.1 $B_{r}(0) \cap \phi_{v^{*}}^{-1}(R) \subset \dot{V}_{p ; S}^{k} \subset \mathcal{B}_{1}\left(Q^{d}\right)$ is relatively compact in the latter space and by Theorem F3 of [BFT-78] is sequentially dense in its closure. Hence, if $v$ belongs to this closure, then there is a sequence $\left\{v_{j}\right\}_{j \in \mathbb{N}} \subset B_{r}(0) \cap \phi_{v^{*}}^{-1}(R)$ pointwise convergent to $v$ on $Q^{d}$. Therefore $\left\{v_{j}\right\}_{j \in \mathbb{N}}$ satisfies the assumptions of the Rosenthal Main theorem [Ro-77] implying that

$$
\lim _{j \rightarrow \infty} \int_{Q^{d}} v_{j} d \mu=\int_{Q^{d}} v d \mu
$$

for all finite signed Borel measures on $Q^{d}$.

In particular, this is true for discrete measures giving rise by functions in $\hat{\ell}_{1}=\left(U_{p}^{k}\right)^{0}$. But $\left(U_{p}^{k}\right)^{0}$ is dense in $U_{p}^{k}$ and by our definition the sequence $\left\{v_{j}\right\}_{j \in \mathbb{N}}$ is bounded in $\dot{V}_{p ; S}^{k}$. Hence, (13.22) implies that

$$
\lim _{j \rightarrow \infty} v_{j}(u)=v(u) \quad \text { for all } \quad u \in U_{p}^{k}
$$

here we regard $v_{j}$ and $v$ as bounded linear functionals on $U_{p}^{k}$, see Theorem 2.25.

This means that $\left\{v_{j}\right\}_{j \in \mathbb{N}} \subset \dot{V}_{p ; S}^{k}$ weak* converges to $v$.

To show weak* closedness of $B_{r}(0) \cap \phi_{v^{*}}^{-1}(R)$ it remains to prove the following:

Lemma 13.9. If a sequence $\left\{v_{j}\right\}_{j \in \mathbb{N}} \subset B_{r}(0) \cap \phi_{v^{*}}^{-1}(R)$ weak* converges to some $v \in V_{p}^{k}$, then $v \in B_{r}(0) \cap \phi_{v^{*}}^{-1}(R)$.

Proof. Weak ${ }^{*}$ convergence of $\left\{v_{j}\right\}_{j \in \mathbb{N}}$ to $v$ implies pointwise convergence of the sequence of functions $\left\{\left.v_{j} \circ \tau\right|_{i^{*}\left(\overline{\mathscr{B}}_{p}^{k}\right)}\right\}_{j \in \mathbb{N}}$ to the function $\left.v \circ \tau\right|_{i^{*}\left(\overline{\mathscr{B}}_{p}^{k}\right)}$, see (13.19). Further, the functions of

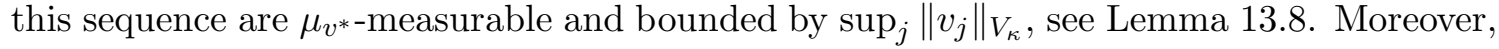
by the assumption of Lemma 13.9

$$
\sup _{j}\left\|v_{j}\right\|_{V_{p}^{k}} \leq r \quad \text { and } \quad \phi_{v^{*}}\left(v_{j}\right) \in R, \quad j \in \mathbb{N} .
$$

Therefore, the Lebesgue pointwise convergence theorem implies

$$
\lim _{j \rightarrow \infty} \phi_{v^{*}}\left(v_{j}\right)=\int_{\mathfrak{i}^{*}\left(\overline{\mathscr{B}}_{p}^{k}\right)}\left(\lim _{j \rightarrow \infty} v_{j} \circ \tau\right) d \mu_{v^{*}}=\int_{\mathfrak{i}^{*}\left(\overline{\mathscr{B}}_{p}^{k}\right)} v \circ \tau d \mu_{v^{*}}=\phi_{v^{*}}(v) .
$$


Since $R \subset \mathbb{R}$ is closed, the limit on the left-hand side belongs to $R$, hence, the limit point $v \in B_{r}(0) \cap \phi_{v^{*}}^{-1}(R)$ as required.

Thus, $\phi_{v^{*}}$ is a weak* continuous linear functional from $\left(V_{p}^{k}\right)^{*}$. By the definition of the weak* topology on $V_{p}^{k}=\left(U_{p}^{k}\right)^{*}$ every weak* continuous functional is uniquely determined by an element of $U_{p}^{k}$, i.e., for some $u_{v^{*}} \in U_{p}^{k}$

$$
\phi_{v^{*}}(v)=v\left(\mathfrak{i}^{*}\left(u_{v^{*}}\right)\right), \quad v \in V_{p}^{k} .
$$

On the other hand, see (13.21), for all $v \in \mathrm{V}_{p}^{k}$,

$$
\phi_{v^{*}}(v)=v\left(v^{*}\right) .
$$

Moreover, since $\left.\mathfrak{i}^{*}\right|_{U_{p}^{k}}: U_{p}^{k} \rightarrow\left(\mathrm{v}_{p}^{k}\right)^{*}$ is an isomorphic embedding, see Proposition 13.1(a), $\mathrm{V}_{p}^{k}$ separates points of $U_{p}^{k}$.

These two equalities imply that

$$
v^{*}=\mathfrak{i}^{*}\left(u_{v^{*}}\right) .
$$

Thus, every point $v^{*} \in\left(\mathrm{v}_{p}^{k}\right)^{*}$ is the image under $\mathfrak{i}^{*}$ of some point of $U_{p}^{k}$, i.e., $\mathfrak{i}^{*}: U_{p}^{k} \rightarrow\left(\mathrm{v}_{p}^{k}\right)^{*}$ is a surjection. Moreover, $\left.\mathfrak{i}^{*}\right|_{U_{p}^{k}}$ is also an embedding. Hence, $\mathfrak{i}^{*}$ is an isomorphism of the Banach spaces $U_{p}^{k}$ and $\left(\mathrm{v}_{p}^{k}\right)^{*}$.

This completes the proof of Theorem 2.27

Proof of Corollary 2.28. The result is the combination of Theorems 2.27 and 2.25 .

\section{REFERENCES}

[AC-33] C. R. Adams and J. A. Clarkson, On definitions of bounded variation for functions of two variables, Trans. Amer. Math. Soc. 34 (1933), 824-854.

[AC-34] C. R. Adams and J. A. Clarkson, Properties of functions $f(x, y)$ of bounded variation, Trans. Amer. Math. Soc. 36 (1934), 711-730.

[AFP-00] L. Ambrosio, N. Fusco, D. Pallara, Functions of Bounded Variations and Discontinuity Problems, Oxford Sci. Publ., 2000.

[BBI-11] A. Brudnyi and Yu. Brudnyi, Methods of Geometric Analysis in Lipschitz Extension and Trace Problems, Volume I, Monographs in Mathematics, Vol. 102, Springer, Basel, 2011.

[BBII-11] A. Brudnyi and Yu. Brudnyi, Methods of Geometric Analysis in Lipschitz Extension and Trace Problems, Volume II, Monographs in Mathematics, Vol. 103, Springer, Basel, 2011.

[BB-18] A. Brudnyi and Yu. Brudnyi, Banach structure of multivariate BV spaces I, Preprint 2018, 48 pps. [BFT-78] J. Bourgain, D. H. Fremlin, and M. Talagrand, Pointwise compact sets of Baire-measurable functions, Amer. J. Math. 100 (1978), 845-886.

[BG-73] Yu. Brudnyi and M. Ganzburg, On an extremal problem for polynomials of $n$ variables, Izv. Acad. Nauk SSSR 37 (1973), 344-355. English transl. in: Math. USSR Izv. 7 (1973), 345-356.

[BH-32] J. C. Burkill and U. S. Haslam-Jones, Notes on the differentiability of functions of two variables, J. London Math. Soc. 7 (1932), 297-305.

[BK-91] Yu. Brudnyi and N. Krugljak, Interpolation Functors and Interpolation Spaces, North Holland, 1991.

[Br1-70] Yu. Brudnyi, A multivariate generalization of a theorem of Whitney, Mat. Sb. 82 (1970), $175-191$. English Transl. in Mat. USSR Sb. 11 (1970), 157-170.

[Br1-71] Yu. Brudnyi, Spaces defined by means of local approximations, Tr. Mosk. Mat. Obs. 24 (1971), 69-132. English Transl. in Moscow Math. Soc. 24 (1971), 73-139. 
[Br1-94] Yu. Brudnyi, Adaptive approximation of functions with singularities, Trudy Mosk. Mat. Ob-va 55 (1994), 149-242. English transl. in : Trans. Moscow Math. Soc. (1994), 23-186.

[Br2-15] A. Brudnyi, Differential calculus on topological spaces with weak Markov structure I, Rev. Mat. Iberoam. 31 (2015), no. 2, 531-574.

[Ca-67] L. Carleson, Selected Problems on Exceptional Sets, Van Nostrand, 1967.

[Ce-36] L. Cezari, Sulla funzioni a variazione limitata, Ann. Scuola Norm. Sup. Pisa Cl. Sci. (2) 5 (1936), 299-313.

[CZ-61] A. P. Calderón and A. Zygmund, Local properties of solutions of elliptic partial differential equations, Studia Math. XX (1961), 171-235.

[De-35] A. Denjoy, Sur l'integration des coefficients diffrentiels d'ordre supérreur, Fund. Math. 25 (1935), 273-326.

[DG-54] E. De Giorgi, Su una teoria generale della misura $(r-1)$ - dimensionale in uno spazio ad $r$ dimensioni, Ann. Mat. Pura Appl. IV 36 (1954), 191-213.

[DSch-58] N. Dunford and J. T. Schwartz, Linear Operators. Part 1, Intersci. Publ., 1958.

[FKP-57] W. H. Federer, K. Krickeberg and C. Y. Pauc, Three papers on summable functions whose first derivatives are measures, Ann. Mat. Pure Appl. (4) 44 (1957), 93-152.

[Ga-59] E. Gagliardo, Ulteriori proprietà di alcune classi di funzioni in più variabili, Ricerche Mat. 8 (1959), $24-51$.

[Ge-54] F. Gehring, Study of $\alpha$-variation, Trans. Amer. Mat. Soc. 76 (1954), 432-443.

[Gi-84] E. Giusti, Minimal Surfaces and Functions of Bounded Variation, Birkhäuser, 1984.

[Gu-75] M. De Guzman, Differentiation of Integrals in $\mathbb{R}^{n}$, Springer, 1975.

[H-06] G. H. Hardy, On double Fourier series and especially those which represent the double zeta-function with real and incommensurable parameters, Quarterly J. Math. 37 (1906) 53-70.

[HL-28] G. H. Hardy and J. E. Littlewood, A convergence criterion for Fourier series, Math. Z. 28 (1928), $612-634$

[JH-77] H. Johnen and K. Scherer, On the equivalence of the $K$-functional and moduli of continuity and some applications, Lect. Notes in Math. 571 (1977), 119-140.

[KL-09] V. Kolyada and M. Lindt, On functions of bounded p-variation, J. Math. Anal. Appl. 356 (2009), $582-604$

[Kr-50] A. S. Kronrod, On functions of two variables, Uspekhi Mat. Nauk, 5, (1) (1950), 24-134 (in Russian).

[Le-10] H. Lebesgue, Sur l'integration de functiones discontinues, Ann. Ecole Norm. Sup. 27 (1910), 361450.

[Ma-64] J. Marcinkiewicz, On a class of functions and their Fourier series, Collected Papers. Warszawa. PAN, 1964, 36-41.

[Ma-85] V. Mazya, Sobolev Spaces, Springer, 1985.

[MT-50] M. Morse and W. Transue, The Fréchet variation a generalization for multiple Fourier series of the Jordan test, Rev. Mat. Univ. Parma 1 (1950), 9-18.

[MZ-36] J. Marcinkiewicz and A. Zygmund, On the differentiability of functions and summability of trigonometric series, Fund. Math. 26 (1936), 1-43.

[Pe-1891] G. Peano, Sulla formula di Taylor, Atti. Acad. Sci. Torino 27 (1891-1892), 40-46.

[Ro-77] H. P. Rosenthal, Point-wise compact subsets of the first Baire class, Amer. J. Math. 99 (1977), 362-378.

[S-37] S. Saks, Theory of Integral, Warsaw, 1937.

[Te-015] V. Temlyakov, Constructive sparse trigonometric approximation and other problems for functions with mixed smoothness, Sb. Math. 206 (2015), 1628-1656.

[T-26] L. Tonelli, Sulla quadratura delle superficie, Atti Accad. Naz. Lincei, Ser. 3 (1926), 357-363, 445-450, 633-658.

[Tr-92] H. Triebel, Function Space Theory. II, Birkhaäuser, 1992. 
[V-05] G. Vitali, Sulle funzioni integrali, Atti. R. Accad. sci. Torino, Cl. Sci Fis. Mat. Natur. 40 (1904-05), 1021-1034.

[Vi-55] A. G. Vitushkin, On Multidimensional Variations, Gosizdat, 1955 (in Russian).

[Vi-004] A. G. Vitushkin, The 13th problem of Hilbert and related problems, Uspekhi Mat. Nauk 59 (1) (2004), 11-24.

[VP-16] Ch. J. Vallée-Poussen, Integrale de Lebesgue, Functions d'Ensemble, Classes de Baire, Paris, 1916.

[Wh-59] H. Whitney, On bounded functions with bounded $n$th differences, Proc. AMS 10 (1959), 480-481.

Department of Mathematics and Statistics

UNIVERSity OF CALGARY

Calgary, Alberta, Canada

$\mathrm{T} 2 \mathrm{~N} 1 \mathrm{~N} 4$

E-mail address: abrudnyi@ucalgary.ca

Department of Mathematics

TECHNION

HAIFA, ISRAEL

32000

E-mail address: ybrudnyi@math.technion.ac.il 